\title{
A Second Order Minimality Condition for a Free-Boundary Problem
}

\author{
Irene Fonseca Giovanni Leoni Maria Giovanna Mora
}

July 2, 2021

\begin{abstract}
The goal of this paper is to derive in the two-dimensional case necessary and sufficient minimality conditions in terms of the second variation for the functional

$$
v \mapsto \int_{\Omega}\left(|\nabla v|^{2}+\chi_{\{v>0\}} Q^{2}\right) d \boldsymbol{x}
$$

introduced in a classical paper of Alt and Caffarelli. For a special choice of $Q$ this includes water waves. The second variation is obtained by computing the second derivative of the functional along suitable variations of the free boundary. It is proved that the strict positivity of the second variation gives a sufficient condition for local minimality. Also, it is shown that smooth critical points are local minimizers in a small tubular neighborhood of the free-boundary.
\end{abstract}

2010 Mathematics Subject Classification: 35R35, 49J40

\section{Introduction}

The goal of this paper is to derive a new minimality condition in terms of the second variation for the functional

$$
\mathcal{F}(v):=\int_{\Omega}\left(|\nabla v|^{2}+\chi_{\{v>0\}} Q^{2}\right) d \boldsymbol{x}, \quad v \in \mathcal{A}_{0},
$$

introduced by Alt and Caffarelli in the seminal paper [AC] (see also [ACF1, ACF2, ACF3, CS, [F]). Here, $\Omega \subset \mathbb{R}^{N}$ is an open connected set with locally Lipschitz boundary, the function $Q: \Omega \rightarrow[0,+\infty)$ is continuous, and

$$
\mathcal{A}_{0}:=\left\{v \in H_{\text {loc }}^{1}(\Omega): \nabla v \in L^{2}\left(\Omega ; \mathbb{R}^{N}\right), v=v^{*} \text { on } S\right\},
$$

where $S \subset \partial \Omega$ is a measurable set with $\mathcal{H}^{n-1}(S)>0$, and the Dirichlet datum $v^{*} \in H_{\mathrm{loc}}^{1}(\Omega)$ is a nonnegative function with $\nabla v^{*} \in L^{2}\left(\Omega ; \mathbb{R}^{N}\right)$. The identity $v=v^{*}$ on $S$ is to be understood in the sense of traces. 
In this paper a critical point for (1.1) is a function $v \in \mathcal{A}_{0}$ such that $\mathcal{F}(v) \in \mathbb{R}$ and

$$
\left.\frac{d \mathcal{F}}{d \varepsilon}(v+\varepsilon \varphi)\right|_{\varepsilon=0}=0
$$

for every $\varphi \in H^{1}(\Omega)$ with $\varphi=0$ on $S$ in the sense of traces. It can be shown that when $v$ is a smooth critical point, e.g., $v \in C^{2}(\bar{\Omega})$, and the free boundary $\Omega \cap \partial\{v>0\}$ is a manifold of class $C^{2}$, then we are led to a free boundary problem (see $\underline{\mathrm{KS}}$ ). To be precise, the Euler-Lagrange equations of (1.1) are given by

$$
\begin{cases}\Delta v=0 & \text { in } \Omega \cap\{v>0\}, \\ v=0, \quad|\nabla v|=Q & \text { on } \Omega \cap \partial\{v>0\}, \\ v=v^{*} & \text { on } S,\end{cases}
$$

(see the Appendix).

Under the assumptions that $Q$ is Hölder continuous and

$$
0<Q_{\min } \leq Q(\mathbf{x}) \leq Q_{\max }<\infty,
$$

Alt and Caffarelli $\mathrm{AC}$ proved existence of global minimizers, full regularity of the free boundary $\Omega \cap \partial\{v>0\}$ of local minimizers for $N=2$ and partial regularity for $N \geq 3$. Using a monotonicity formula, Weiss in [W] improved the estimate of the Hausdorff dimension of the singular set, and Caffarelli, Jerison, and Kenig [CJK showed full regularity in dimension $N=3$. Note, however, that in dimension $N=3$ there exist critical points of (1.1) whose free boundary is singular (see [AC] and CJK]).

In this work we prove that in dimension $N=2$ and under the assumption (1.4), smooth critical points of (1.1) are actually local minimizers with respect to small $C^{2, \alpha}$ perturbations (see the statement of Theorem 1.2 for the precise notion of minimality) in a tubular neighborhood of $\partial\{u>0\} \cap \Omega$. The proof is based on the derivation of a second order variation of the functional (1.1).

This approach has been successfully applied to several contexts. In particular, in the study of the Mumford-Shah functional the strict positivity of the second variation has been used to obtain local minimality of critical points (see [BM], CMM], [C, $\mathrm{MM}$ ), including triple junctions, which are at the core of the Mumford-Shah conjecture. Furthermore, using the diffuse-interface Ohta-Kawasaki energy to model microphase separation in diblock copolymer melts, critical configurations with positive second variation were found to be local minimizers in [AFM] (see also [BC1, $\mathrm{BC} 2)$. In turn, these results are used to determine global and local minimality of certain lamellar configurations. Finally, in $[\mathrm{FM}$ (see also $[\mathrm{B}$ ) the authors analyzed a variational model for epitaxial growth of a thin elastic film over a flat substrate when a lattice mismatch between the two materials is present. Again using techniques involving the positivity of the second variation, they determined the critical threshold for local and global minimality of the flat configuration.

We now present the main results of this paper. We assume $\Omega:=(-1,1) \times(0, \infty)$, and we consider the functional $\mathcal{F}$ in (1.1) defined on the class

$$
\begin{aligned}
\mathcal{A}:=\left\{v \in L_{\mathrm{loc}}^{1}(\Omega): \nabla v \in L^{2}\left(\Omega ; \mathbb{R}^{2}\right), v(x, 0)\right. & =u^{*}(x) \text { for } x \in(-1,1), \\
& v(-1, y)=v(1, y) \text { for } y>0\},
\end{aligned}
$$


where $\mathbf{x}=(x, y) \in \mathbb{R}^{2}, u^{*} \in C^{1}([-1,1])$ is periodic, and $u^{*}>0$, while the function $Q$ satisfies

$$
Q \in C^{0,1}(\Omega), \quad 0 \leq Q(\boldsymbol{x}) \leq Q_{\max } \quad \text { for every } \boldsymbol{x} \in \Omega .
$$

By Theorem 1.3 in $[\mathrm{AC}$ (see also $\mathrm{AL}$ ), there exists a minimizer of $\mathcal{F}$ in $\mathcal{A}$. Moreover, in view of Lemma 2.4 in $[\mathrm{AC}$, for any local minimizer $v$ of $\mathcal{F}$ in $\mathcal{A}$, the set $\{v>0\} \cap \Omega$ is open and $v$ is harmonic in $\{v>0\} \cap \Omega$. Let $u \in \mathcal{A}$ be such that the set

$$
\Omega_{+}:=\{u>0\} \cap \Omega
$$

is open, $u$ is harmonic in $\Omega_{+}$, and

$$
\Gamma:=\partial\{u>0\} \cap \Omega
$$

is a smooth curve. Then $u$ satisfies the elliptic problem

$$
\begin{cases}\Delta u=0 & \text { in } \Omega_{+}, \\ u=0 & \text { on } \Gamma, \\ u=u^{*} & \text { on }\{y=0\} \cap \partial \Omega_{+},\end{cases}
$$

together with the periodicity conditions

$$
u(-1, y)=u(1, y) \text { for } y>0 .
$$

We consider a one-parameter family of diffeomorphisms $\left\{\Phi_{s}\right\}_{s \in[0,1]}$ that coincide with the identity in a uniform neighborhood of $\partial \Omega$. We then derive the second derivative of $\mathcal{F}\left(u_{s}\right)$ with respect to $s$, where $u_{s}$ is the minimizer of the Dirichlet energy on $\Phi_{s}(\{u>0\})$ with respect to the given boundary conditions. Imposing the first derivative of $\mathcal{F}\left(u_{s}\right)$ to be zero at $s=0$ gives back the equilibrium condition $|\nabla u|=Q$ on $\Omega \cap \partial\{u>0\}$. The second order derivative of $\mathcal{F}\left(u_{s}\right)$ provides a new necessary condition for minimizers, expressed in terms of a sign condition for a quadratic form (see Remark 2.4 below). In turn, the strict positivity of this quadratic form gives a sufficient condition for local minimality. This is made precise by the following theorem, which is one of the main results of this paper.

In what follows, we denote by $\nu: \Gamma \rightarrow \mathbb{S}^{1}$ a smooth normal vector to $\Gamma$. The curvature $\kappa$ of $\Gamma$ satisfies $\partial_{\tau} \nu=\kappa \tau$ and $\partial_{\tau} \tau=-\kappa \nu$, where $\tau: \Gamma \rightarrow \mathbb{S}^{1}$ is a smooth tangent vector to $\Gamma$.

Theorem 1.1 Assume that $Q \in C^{1,1}(\Omega)$ and satisfies (1.6). Let $u \in C^{2, \alpha}\left(\Omega_{+} \cup \Gamma\right)$, $\alpha>0$, satisfy (1.7), (1.9), (1.10), and let the free boundary $\Gamma$ given in (1.8) be the graph of a $C^{3}$ periodic function. Assume, in addition, that

$$
\left(\partial_{\nu} u\right)^{2}=Q^{2} \quad \text { on } \Gamma
$$

and that there exists $C_{0}>0$ such that

$$
\int_{\Omega_{+}} 2\left|\nabla u_{\psi}\right|^{2} d \boldsymbol{x}+\int_{\Gamma}\left(\partial_{\nu} Q^{2}+2 \kappa Q^{2}\right) \psi^{2} d \mathcal{H}^{1} \geq C_{0}\|\psi\|_{H^{1 / 2}(\Gamma)}^{2}
$$


for every $\psi \in C_{c}^{1}(\Gamma)$, where $u_{\psi}$ is the solution to

$$
\begin{cases}\Delta u_{\psi}=0 & \text { in } \Omega_{+}, \\ u_{\psi}=Q \psi & \text { on } \Gamma, \\ u_{\psi}=0 & \text { on }\{y=0\} \cap \partial \Omega_{+},\end{cases}
$$

with $u_{\psi}(-1, y)=u_{\psi}(1, y)$ for all $y$ such that $( \pm 1, y) \in \overline{\Omega_{+}}$. Then there exists $\delta>0$ such that for every open set $U \Subset \Omega$ and for every diffeomorphism $\Phi \in C^{2, \alpha}\left(\mathbb{R}^{2} ; \mathbb{R}^{2}\right)$ with

$$
\operatorname{supp}(\Phi-\mathrm{Id}) \subset U
$$

and

$$
\|\Phi-\mathrm{Id}\|_{C^{2, \alpha}\left(\mathbb{R}^{2}\right)} \leq \delta
$$

we have

$$
\mathcal{F}(u) \leq \mathcal{F}(v)
$$

for every $v \in \mathcal{A}$ with $\{v>0\}=\Phi(\{u>0\})$.

Although the notion of $C^{2, \alpha}$-minimality established in the previous theorem may be perceived as weak, it has been shown to lead to a stronger minimality property in several of the contexts mentioned above. To be precise, in the case of epitaxial growth Fusco and Morini FM proved that the strict positivity of the second variation implies local minimality with respect to $W^{2, \infty}$ perturbations and in turn, that this leads to local minimality with respect to $L^{\infty}$ perturbations. Similarly, for the diffuseinterface Ohta-Kawasaki energy it is shown in AFM that the strict positivity of the second variation yields local minimality with respect to $W^{2, p}$ perturbations and that $W^{2, p}$-local minimizers are actually $L^{1}$-local minimizers. Thus, it is natural to expect that in our setting $C^{2, \alpha}$-minimizers are in fact local minimizers in a much larger class of competitors. This will be addressed in a forthcoming paper.

We also observe that a different type of second variation for the functional (1.1) has been used by Caffarelli, Jerison, and Kenig in CJK to prove full regularity of global minimizers when $N=3$, and by Weiss and Zhang in WZ for a similar functional related to water waves with vorticity. In contrast to our case, where we perform variations of the free boundary $\Gamma$, in $[\mathrm{CJK}$ and $\mathrm{WZ}$ the variations are of the type $u+\varepsilon v$, where $v$ is harmonic in $\Omega_{+} \cap B$ with boundary datum a given function $g$ on $\partial\left(\Omega_{+} \cap B\right)$ and $B$ is a ball.

In the second main theorem we prove that, if $u$ is a smooth critical point of $\mathcal{F}$ restricted to $\mathcal{A}$, then $u$ satisfies the minimality property of Theorem 1.1 in a tubular neighborhood of $\Gamma$.

Theorem 1.2 Assume that $Q$ satisfies (1.6) and $Q \geq Q_{\min }>0$. Let $u \in C^{2, \alpha}\left(\Omega_{+}\right)$ be as in (1.7)-(1.10), and let $\Gamma$ be the graph of a $C^{3}$ periodic function. Assume, in addition, that

$$
\left(\partial_{\nu} u\right)^{2}=Q^{2} \quad \text { on } \Gamma .
$$

Then there exist $\varepsilon>0$ and $c_{\varepsilon}>0$ such that

$$
\int_{U_{\varepsilon} \cap\{u>0\}} 2\left|\nabla u_{\psi}\right|^{2} d \boldsymbol{x}+\int_{\Gamma}\left(\partial_{\nu} Q^{2}+2 \kappa Q^{2}\right) \psi^{2} d \mathcal{H}^{1} \geq c_{\varepsilon}\|\psi\|_{H^{1 / 2}(\Gamma)}^{2}
$$


for every $\psi \in C_{c}^{1}(\Gamma)$, where $U_{\varepsilon}$ is the intersection of $\Omega$ with the $\varepsilon$-tubular neighborhood of $\Gamma$. In particular, if $Q \in C^{1,1}(\Omega)$ then there exists $\delta_{\varepsilon}>0$ such that for every open set $V_{\varepsilon} \Subset U_{\varepsilon}$ and for every diffeomorphism $\Phi \in C^{2, \alpha}\left(\mathbb{R}^{2} ; \mathbb{R}^{2}\right)$ with

$$
\operatorname{supp}(\Phi-\mathrm{Id}) \subset V_{\varepsilon} \quad \text { and } \quad\|\Phi-\mathrm{Id}\|_{C^{2, \alpha}\left(\mathbb{R}^{2}\right)} \leq \delta_{\varepsilon},
$$

we have

$$
\int_{U_{\varepsilon}}\left(|\nabla u|^{2}+\chi_{\{u>0\}} Q^{2}\right) d \boldsymbol{x} \leq \int_{U_{\varepsilon}}\left(|\nabla v|^{2}+\chi_{\{v>0\}} Q^{2}\right) d \boldsymbol{x}
$$

for every $v \in L_{\text {loc }}^{1}\left(U_{\varepsilon}\right)$ such that $\nabla v \in L^{2}\left(U_{\varepsilon} ; \mathbb{R}^{2}\right), v=u$ on $\partial U_{\varepsilon} \cap \Omega_{+}, v(-1, y)=$ $v(1, y)$ for all $y$ such that $( \pm 1, y) \in \partial U_{\varepsilon}$, and $\{v>0\}=\Phi(\{u>0\})$.

The constant $c_{\varepsilon}$ in (1.15) depends strongly on $Q_{\min }$. This is not surprising, since the hypothesis $Q_{\min }>0$ is fundamental for the regularity of local minimizers. When $Q_{\min }=0$ one expects the free boundary to present singularities at points where $Q(\mathbf{x})=0$. Indeed, in dimension $N=2$ and when $Q(x, y)=\sqrt{(q-2 g y)_{+}}$, where $q$ is a physical constant related to the hydraulic head and $g$ is the gravitational acceleration, the free boundary problem (1.3) is related to Stokes waves of greatest height, which are characterized by the fact that their shape is not regular but has a sharp crest of included angle $\frac{2}{3} \pi$ (see, e.g., [AL, [CoSS], CoS], MT, [PT], [ST], [S], [S2, [T, [V], VW], VW2, [W], WZ, and the references therein).

This paper is organized as follows. In Section 2 we give the precise definition of admissible flows and derive the second variation of the functional (1.1). In Section 3 , given a small perturbation of $\Gamma$, we construct an admissible flow (see Definition 2.1) joining $\Gamma$ to the perturbed free boundary and with zero tangential velocity on the free boundaries. This latter property will play a crucial role in the proofs of the main theorems, and it leads to a first order partial differential equation (see (3.41) below) that we solve using the method of characteristics. One of the main difficulties is that the components of the flow are given by compositions of functions that are discontinuous. Thus, proving the regularity of the flow is extremely delicate and it will be carried out in the appendix. In Section 4 we prove Theorem [1.1 To control the second variation along the flow we use sharp Schauder estimates together with the zero tangential velocity of the flow. Finally, in Section 5 we prove Theorem 1.2 .

\section{The Second Variation}

In this section we derive the second variation of $\mathcal{F}$ on some suitable variations of $u$ that are constructed along a family of variations of $\Gamma$ according to the following definition.

Definition 2.1 We say that $\left\{\Phi_{s}\right\}_{s \in[0,1]}$ is an admissible flow if it satisfies the following conditions:

(i) the map $(s, \boldsymbol{x}) \mapsto \Phi_{s}(\boldsymbol{x})$ belongs to $C^{2}\left([0,1] \times \bar{\Omega} ; \mathbb{R}^{2}\right)$;

(ii) for every $s \in[0,1]$, the map $\Phi_{s}$ is a diffeomorphism from $\bar{\Omega}$ onto itself; 
(iii) $\Phi_{0}=\operatorname{Id}$ in $\bar{\Omega}$;

(iv) there exists an open set $U$, compactly contained in $\Omega$, such that $\operatorname{supp}\left(\Phi_{s}-\mathrm{Id}\right) \subset$ $U$ for all $s \in[0,1]$.

Let $\left\{\Phi_{s}\right\}_{s \in[0,1]}$ be an admissible flow, and let $u$ be as in Theorem 1.1] For every $s \in[0,1]$ we consider the solution $u_{s}$ of the problem

$$
\begin{cases}\Delta u_{s}=0 & \text { in } \Phi_{s}\left(\Omega_{+}\right), \\ u_{s}=0 & \text { on } \Phi_{s}(\Gamma), \\ u_{s}=u & \text { on } \partial \Phi_{s}\left(\{y=0\} \cap \partial \Omega_{+}\right),\end{cases}
$$

with $u_{s}(-1, y)=u_{s}(1, y)$ for all $y$ such that $( \pm 1, y) \in \Phi_{s}\left(\overline{\Omega_{+}}\right)$. Note that, in view of property (iv) in Definition 2.1] we have that

$$
\partial \Phi_{s}\left(\{y=0\} \cap \partial \Omega_{+}\right)=\{y=0\} \cap \partial \Omega_{+}
$$

and $( \pm 1, y) \in \Phi_{s}\left(\overline{\Omega_{+}}\right)$if and only if $( \pm 1, y) \in \overline{\Omega_{+}}$. Moreover, extending $u_{s}$ by 0 outside $\Phi_{s}\left(\Omega_{+}\right)$, we obtain $u_{s} \in \mathcal{A}$.

In what follows, for every $s \in[0,1]$ and $\boldsymbol{x} \in \Omega$ we denote by $\dot{u}_{s}(\boldsymbol{x})$ the partial derivative with respect to $r$ of the function $(r, \boldsymbol{x}) \mapsto u_{r}(\boldsymbol{x})$ evaluated at $(s, \boldsymbol{x})$, that is,

We define

$$
\dot{u}_{s}(\boldsymbol{x}):=\left.\frac{\partial u_{r}}{\partial r}(\boldsymbol{x})\right|_{r=s} \text {. }
$$

$$
X_{s}:=\dot{\Phi}_{s} \circ \Phi_{s}^{-1}, \quad Z_{s}:=\ddot{\Phi}_{s} \circ \Phi_{s}^{-1}
$$

for every $s \in[0,1]$, where

$$
\dot{\Phi}_{s}:=\left.\frac{\partial \Phi_{r}}{\partial r}\right|_{r=s}, \quad \ddot{\Phi}_{s}:=\left.\frac{\partial^{2} \Phi_{r}}{\partial r^{2}}\right|_{r=s} .
$$

Moreover, we set $\Gamma_{s}:=\Phi_{s}(\Gamma)$ and denote by $\tau_{s}$ and $\nu_{s}$ the tangent and normal vector to $\Gamma_{s}$ given by

$$
\tau_{s}:=\frac{\left(D \Phi_{s}\right) \tau}{\left|\left(D \Phi_{s}\right) \tau\right|} \circ \Phi_{s}^{-1}, \quad \nu_{s}:=\frac{\left(D \Phi_{s}\right)^{-T} \nu}{\left|\left(D \Phi_{s}\right)^{-T} \nu\right|} \circ \Phi_{s}^{-1}
$$

Finally, $\kappa_{s}$ denotes the curvature of $\Gamma_{s}$.

The proof of the following proposition follows the arguments in CMM].

Proposition 2.2 Let $u \in C^{2}\left(\Omega_{+} \cup \Gamma\right)$ satisfy 1.7)-(1.10), let $\left\{\Phi_{s}\right\}_{s \in[0,1]}$ be an admissible flow, and let $\hat{u}_{s}:=u_{s} \circ \Phi_{s}$, where $u_{s}$ solves (2.1). Then the map

$$
s \mapsto \hat{u}_{s}
$$

belongs to $C^{1}\left([0,1] ; H^{1}\left(\Omega_{+}\right)\right)$. In particular, the function $\dot{u}_{s}$ in (2.2) is well-defined and is the unique solution to the boundary value problem

$$
\begin{cases}\Delta \dot{u}_{s}=0 & \text { in } \Phi_{s}\left(\Omega_{+}\right) \\ \dot{u}_{s}=-\left(X_{s} \cdot \nu_{s}\right) \partial_{\nu_{s}} u_{s} & \text { on } \Gamma_{s} \\ \dot{u}_{s}=0 & \text { on } \partial \Phi_{s}\left(\{y=0\} \cap \partial \Omega_{+}\right),\end{cases}
$$


with $\dot{u}_{s}(-1, y)=\dot{u}_{s}(1, y)$ for all $y$ such that $( \pm 1, y) \in \Phi_{s}\left(\overline{\Omega_{+}}\right)$.

Proof. For simplicity, we only prove the result in a neighborhood of $s=0$. The general case can be obtained analogously. In view of (2.1) a straightforward computation shows that $\hat{u}_{s}$ satisfies

$$
\begin{cases}\operatorname{div}\left(A_{s} \nabla \hat{u}_{s}\right)=0 & \text { in } \Omega_{+}, \\ \hat{u}_{s}=u & \text { on } \Gamma \cup\left(\{y=0\} \cap \partial \Omega_{+}\right),\end{cases}
$$

with $\hat{u}_{s}(-1, y)=\hat{u}_{s}(1, y)$ for all $( \pm 1, y) \in \overline{\Omega_{+}}$, where

$$
A_{s}:=\left(\frac{D \Phi_{s}^{-1}\left(D \Phi_{s}^{-1}\right)^{T}}{\operatorname{det} D \Phi_{s}^{-1}}\right) \circ \Phi_{s} .
$$

Let $V$ be the subspace of all functions $v \in H^{1}\left(\Omega_{+}\right)$such that $v=0$ on $\Gamma \cup(\{y=$ $\left.0\} \cap \partial \Omega_{+}\right)$and $v(-1, y)=v(1, y)$ for all $( \pm 1, y) \in \overline{\Omega_{+}}$. For every $s \in[0,1]$ and $v \in V$ let $\mathcal{H}(s, v)$ be the unique weak solution $w \in V$ of the Poisson's equation

$$
\Delta w=\operatorname{div}\left(A_{s} \nabla(v+u)\right) \quad \text { in } \Omega_{+} .
$$

Then

$$
\mathcal{H}:[0,1] \times V \rightarrow V
$$

Observe that $A_{0}=I_{2 \times 2}$ and $u$ is harmonic in $\Omega_{+}$by (1.9), hence $\mathcal{H}(0,0)=0$. Moreover, (1.9) implies that $\mathcal{H}(0, v)=v$, thus $\partial_{v} \mathcal{H}(0,0)$ is the identity operator from $V$ into $V$. Since the matrix $A_{s}$ in (2.8) is of class $C^{1}$, by standard elliptic estimates (see also the proof of Proposition 4.2 below), we have that the map $\mathcal{H}$ is of class $C^{1}$. Hence, we are in a position to apply the implicit function theorem (see, e.g., AP. Theorem 2.3]) to find $\delta_{0}>0$ and $r_{0}>0$ and a unique continuous function $g:\left[0, \delta_{0}\right] \rightarrow B_{V}\left(0, r_{0}\right)$ such that $g(0)=0$ and

$$
\mathcal{H}(s, g(s))=0
$$

for all $s \in\left[0, \delta_{0}\right]$. Moreover, $g$ is of class $C^{1}$.

On the other hand, in view of (2.7) the function $\hat{u}_{s}-u$ belongs to $V$ and satisfies

$$
\mathcal{H}\left(s, \hat{u}_{s}-u\right)=0
$$

for all $s \in[0,1]$. Since the map $s \mapsto \hat{u}_{s}-u$ is continuous (see, e.g., the proof of (4.3) below), it follows by uniqueness that $g(s)=\hat{u}_{s}-u$ for all $s \in\left[0, \delta_{0}\right]$. In particular, $s \mapsto \hat{u}_{s}-u$ is of class $C^{1}$.

To prove (2.6), let $v \in H_{\mathrm{loc}}^{1}(\Omega)$ be such that $\nabla v \in L^{2}\left(\Omega ; \mathbb{R}^{2}\right), v(-1, y)=v(1, y)$ for $y \in(0, \infty), v(x, 0)=0$ for $x \in(-1,1)$, and $\bar{\Gamma} \cap \operatorname{supp} v=\emptyset$. Then $\bar{\Gamma}_{s} \cap \operatorname{supp} v=\emptyset$ for all $s$ sufficiently small. By (2.1) it follows that there exists an open subset of $\Omega$ containing $\operatorname{supp} v$ and on which $u_{s}$ is harmonic for all $s$ sufficiently small; thus,

$$
\int_{\Omega} \nabla u_{s} \cdot \nabla v d \boldsymbol{x}=0
$$


for all $s$ sufficiently small. Differentiating the previous identity with respect to $s$ (see (2.2) ) we obtain

$$
\int_{\Omega} \nabla \dot{u}_{s} \cdot \nabla v d \boldsymbol{x}=0
$$

By (2.1) we have that $u_{s}\left(\Phi_{s}(\boldsymbol{x})\right)=0$ for $\boldsymbol{x} \in \Gamma$; thus,

$$
\dot{u}_{s}\left(\Phi_{s}(\boldsymbol{x})\right)=-\nabla u_{s}\left(\Phi_{s}(\boldsymbol{x})\right) \cdot \dot{\Phi}_{s}(\boldsymbol{x}) \quad \text { for } \boldsymbol{x} \in \Gamma,
$$

which by (2.3) is equivalent to

$$
\dot{u}_{s}=-\nabla u_{s} \cdot X_{s} \quad \text { on } \Gamma_{s} .
$$

On the other hand, since $\nabla u_{s}=\partial_{\nu_{s}} u_{s} \nu_{s}$ on $\Gamma_{s}$ by (2.1), we have that $\nabla u_{s} \cdot X_{s}=$ $\left(X_{s} \cdot \nu_{s}\right) \partial_{\nu_{s}} u_{s}$ on $\Gamma_{s}$. In conclusion,

$$
\dot{u}_{s}=-\left(X_{s} \cdot \nu_{s}\right) \partial_{\nu_{s}} u_{s} \quad \text { on } \Gamma_{s} .
$$

Let now $v \in H_{\text {loc }}^{1}(\Omega)$ be such that $\nabla v \in L^{2}\left(\Omega ; \mathbb{R}^{2}\right), v(-1, y)=v(1, y)$ for $y \in$ $(0, \infty), v(x, 0)=0$ for $x \in(-1,1)$, and decompose $v=v_{1}+v_{2}$, where $\bar{\Gamma} \cap \operatorname{supp} v_{1}=\emptyset$. Then by (2.9) and (2.11), integrating by parts we get

$$
\begin{aligned}
\int_{\Omega} \nabla \dot{u}_{s} \cdot \nabla v d \boldsymbol{x} & =\int_{\Omega} \nabla \dot{u}_{s} \cdot \nabla v_{2} d \boldsymbol{x}=\int_{\Gamma_{s}}\left(X_{s} \cdot \nu_{s}\right) \partial_{\nu_{s}} u_{s} \partial_{\nu_{s}} v_{2} d \mathcal{H}^{1} \\
& =\int_{\Gamma_{s}}\left(X_{s} \cdot \nu_{s}\right) \partial_{\nu_{s}} u_{s} \partial_{\nu_{s}} v d \mathcal{H}^{1}
\end{aligned}
$$

for all $s$ sufficiently small. This proves that the function $\dot{u}_{s}$ is a solution to (2.6).

In view of Proposition 2.2 we can now derive the second derivative of $\mathcal{F}\left(u_{s}\right)$.

Theorem 2.3 Let $u \in C^{2}\left(\Omega_{+} \cup \Gamma\right)$ satisfy (1.7)-(1.10), let $Q$ satisfy (1.6), and let $\left\{\Phi_{s}\right\}_{s \in[0,1]}$ be an admissible flow. Then

$$
\frac{d}{d s} \mathcal{F}\left(u_{s}\right)=\int_{\Gamma_{s}}\left(Q^{2}-\left|\nabla u_{s}\right|^{2}\right)\left(X_{s} \cdot \nu_{s}\right) d \mathcal{H}^{1}
$$

and

$$
\begin{aligned}
\frac{d^{2}}{d s^{2}} \mathcal{F}\left(u_{s}\right)= & \int_{\Phi_{s}\left(\Omega_{+}\right)} 2\left|\nabla \dot{u}_{s}\right|^{2} d \boldsymbol{x}+\int_{\Gamma_{s}}\left(\partial_{\nu_{s}} Q^{2}+2 \kappa_{s}\left(\partial_{\nu_{s}} u_{s}\right)^{2}\right)\left(X_{s} \cdot \nu_{s}\right)^{2} d \mathcal{H}^{1} \\
& +\int_{\Gamma_{s}}\left(Q^{2}-\left|\nabla u_{s}\right|^{2}\right)\left(Z_{s} \cdot \nu_{s}-2\left(X_{s} \cdot \tau_{s}\right) \partial_{\tau_{s}}\left(X_{s} \cdot \nu_{s}\right)+\kappa_{s}\left|X_{s}\right|^{2}\right) d \mathcal{H}^{1}
\end{aligned}
$$

where $\dot{u}_{s}$ is given in (2.6). 
Remark 2.4 If $u$ is a minimizer of $\mathcal{F}$, then the expression in (2.12) is equal to zero at $s=0$; since this is true for any choice of the admissible flow, this implies

$$
|\nabla u|^{2}=Q^{2} \quad \text { on } \Gamma .
$$

In turn, the second variation at $s=0$ reduces to

$$
\left.\frac{d^{2}}{d s^{2}} \mathcal{F}\left(u_{s}\right)\right|_{s=0}=\int_{\Omega_{+}} 2\left|\nabla u_{X_{0} \cdot \nu}\right|^{2} d \boldsymbol{x}+\int_{\Gamma}\left(\partial_{\nu} Q^{2}+2 \kappa Q^{2}\right)\left(X_{0} \cdot \nu\right)^{2} d \mathcal{H}^{1}
$$

where $u_{X_{0} \cdot \nu}$ is the solution to

$$
\begin{cases}\Delta u_{X_{0} \cdot \nu}=0 & \text { in } \Omega_{+}, \\ u_{X_{0} \cdot \nu}=Q\left(X_{0} \cdot \nu\right) & \text { on } \Gamma, \\ u_{X_{0} \cdot \nu}=0 & \text { on }\{y=0\} \cap \partial \Omega_{+},\end{cases}
$$

with $u_{X_{0} \cdot \nu}(-1, y)=u_{X_{0} \cdot \nu}(1, y)$ for all $y$ such that $( \pm 1, y) \in \overline{\Omega_{+}}$. Indeed, on $\Gamma$ we have $\left(\partial_{\nu} u\right)^{2}=|\nabla u|^{2}=Q^{2}$ and $\partial_{\nu} u<0$ by the Hopf Lemma. Moreover, the expression in (2.15) is nonnegative.

Note that every minimizer $u$ satisfies the necessary condition

$$
\int_{\Omega_{+}} 2\left|\nabla u_{\psi}\right|^{2} d \boldsymbol{x}+\int_{\Gamma}\left(\partial_{\nu} Q^{2}+2 \kappa Q^{2}\right) \psi^{2} d \mathcal{H}^{1} \geq 0
$$

for every $\psi \in C_{c}^{2}(\Gamma)$, where $u_{\psi}$ solves (2.16) with $\psi$ in place of $X_{0} \cdot \nu$. In fact, for every $\psi \in C_{c}^{2}(\Gamma)$ with small $C^{2}$ norm it is possible to construct an admissible flow $\left\{\Phi_{s}\right\}_{s \in[0,1]}$ such that $X_{0} \cdot \nu=\psi$ on $\Gamma$. To see this, it is enough to consider

$$
\Phi_{s}(x, y):=(x, y)+\lambda(y) s \psi(x, w(x)) \nu(x, y),
$$

where the normal $\nu$ to $\Gamma$ has been extended smoothly, and $\lambda$ is a cut-off function (see (3.38) below for more details). Hence, from (2.15) we deduce that 2.17) holds for every $\psi \in C_{c}^{2}(\Gamma)$ with small $C^{2}$ norm. In turn, given an arbitrary $\psi \in C_{c}^{2}(\Gamma)$, using a scaling argument, it can be shown that 2.17) continues to hold.

Remark 2.5 Observe that if $u$ is a critical point of $\mathcal{F}$, that is, u satisfies 2.14) in addition to (1.7)-(1.10), then (2.15) holds.

Proof of Theorem 2.3. In view of (2.1), we have that $u_{s}>0$ in $\Phi_{s}\left(\Omega_{+}\right)$; thus,

$$
\begin{aligned}
\mathcal{F}\left(u_{s}\right) & =\int_{\Phi_{s}\left(\Omega_{+}\right)}\left(\left|\nabla u_{s}\right|^{2}+Q^{2}(\boldsymbol{x})\right) d \boldsymbol{x} \\
& =\int_{\Omega_{+}}\left(\left|\nabla u_{s}\left(\Phi_{s}(\boldsymbol{y})\right)\right|^{2}+Q^{2}\left(\Phi_{s}(\boldsymbol{y})\right)\right) \operatorname{det} D \Phi_{s}(\boldsymbol{y}) d \boldsymbol{y}
\end{aligned}
$$


Differentiating the previous identity with respect to $s$ we obtain

$$
\begin{aligned}
\frac{d}{d s} \mathcal{F}\left(u_{s}\right)= & \int_{\Omega_{+}} 2 \nabla u_{s}\left(\Phi_{s}(\boldsymbol{y})\right) \cdot\left(\nabla \dot{u}_{s}\left(\Phi_{s}(\boldsymbol{y})\right)+D^{2} u_{s}\left(\Phi_{s}(\boldsymbol{y})\right) \dot{\Phi}_{s}(\boldsymbol{y})\right) \operatorname{det} D \Phi_{s}(\boldsymbol{y}) d \boldsymbol{y} \\
& +\int_{\Omega_{+}} \nabla Q^{2}\left(\Phi_{s}(\boldsymbol{y})\right) \cdot \dot{\Phi}_{s}(\boldsymbol{y}) \operatorname{det} D \Phi_{s}(\boldsymbol{y}) d \boldsymbol{y} \\
& +\int_{\Omega_{+}}\left(\left|\nabla u_{s}\left(\Phi_{s}(\boldsymbol{y})\right)\right|^{2}+Q^{2}\left(\Phi_{s}(\boldsymbol{y})\right)\right) \frac{d}{d s}\left(\operatorname{det} D \Phi_{s}(\boldsymbol{y})\right) d \boldsymbol{y}
\end{aligned}
$$

where we used (2.2) and (2.4). By [G, Chapter III, Section 10] we have

$$
\frac{d}{d s}\left(\operatorname{det} D \Phi_{s}\right)=\left[\operatorname{div}\left(\dot{\Phi}_{s} \circ \Phi_{s}^{-1}\right) \circ \Phi_{s}\right] \operatorname{det} D \Phi_{s},
$$

thus, recalling that $X_{s}=\dot{\Phi}_{s} \circ \Phi_{s}^{-1}($ see (2.3) $)$,

$$
\begin{aligned}
\frac{d}{d s} \mathcal{F}\left(u_{s}\right)= & \int_{\Phi_{s}\left(\Omega_{+}\right)} 2 \nabla u_{s} \cdot \nabla \dot{u}_{s} d \boldsymbol{x}+\int_{\Phi_{s}\left(\Omega_{+}\right)} 2 D^{2} u_{s} \nabla u_{s} \cdot X_{s} d \boldsymbol{x} \\
& +\int_{\Phi_{s}\left(\Omega_{+}\right)}\left|\nabla u_{s}\right|^{2} \operatorname{div} X_{s} d \boldsymbol{x}+\int_{\Phi_{s}\left(\Omega_{+}\right)}\left(\nabla Q^{2} \cdot X_{s}+Q^{2} \operatorname{div} X_{s}\right) d \boldsymbol{x} .
\end{aligned}
$$

Integrating by parts, from (2.6) and the fact that $\operatorname{supp}\left(\Phi_{s}-\mathrm{Id}\right) \subset U$ for all $s \in[0,1]$, we deduce that

$$
\begin{aligned}
\frac{d}{d s} \mathcal{F}\left(u_{s}\right) & =\int_{\Gamma_{s}} 2 \dot{u}_{s} \partial_{\nu_{s}} u_{s} d \mathcal{H}^{1}+\int_{\Phi_{s}\left(\Omega_{+}\right)} \operatorname{div}\left(\left(\left|\nabla u_{s}\right|^{2}+Q^{2}\right) X_{s}\right) d \boldsymbol{x} \\
& =\int_{\Gamma_{s}}\left(-2\left(X_{s} \cdot \nu_{s}\right)\left(\partial_{\nu_{s}} u_{s}\right)^{2}+\left(\left|\nabla u_{s}\right|^{2}+Q^{2}\right)\left(X_{s} \cdot \nu_{s}\right)\right) d \mathcal{H}^{1} .
\end{aligned}
$$

Since $\left(\partial_{\nu_{s}} u_{s}\right)^{2}=\left|\nabla u_{s}\right|^{2}$ on $\Gamma_{s}$, we obtain (2.12).

We now derive the second derivative of $\mathcal{F}\left(u_{s}\right)$ with respect to $s$ at $s=0$. First, by the area formula we can write the first derivative as

$$
\frac{d}{d s} \mathcal{F}\left(u_{s}\right)=\int_{\Gamma}\left(Q^{2}\left(\Phi_{s}(\boldsymbol{y})\right)-\left|\nabla u_{s}\left(\Phi_{s}(\boldsymbol{y})\right)\right|^{2}\right) \dot{\Phi}_{s}(\boldsymbol{y}) \cdot \nu_{s}\left(\Phi_{s}(\boldsymbol{y})\right) J_{\Phi_{s}}(\boldsymbol{y}) d \mathcal{H}^{1}(\boldsymbol{y}),
$$

where $J_{\Phi_{s}}:=\left|\left(D \Phi_{s}\right)^{-T} \nu\right| \operatorname{det} D \Phi_{s}$ is the one-dimensional Jacobian of $\Phi_{s}$. Differentiating with respect to $s$ yields

$$
\begin{aligned}
\left.\frac{d^{2}}{d s^{2}} \mathcal{F}\left(u_{s}\right)\right|_{s=0}= & \int_{\Gamma}\left(\nabla Q^{2} \cdot \dot{\Phi}_{0}\right) \dot{\Phi}_{0} \cdot \nu d \mathcal{H}^{1} \\
& -\int_{\Gamma} 2\left(\nabla u \cdot \nabla \dot{u}_{0}+D^{2} u \nabla u \cdot \dot{\Phi}_{0}\right) \dot{\Phi}_{0} \cdot \nu d \mathcal{H}^{1} \\
& +\left.\int_{\Gamma}\left(Q^{2}-|\nabla u|^{2}\right) \frac{d}{d s}\left[\dot{\Phi}_{s} \cdot\left(\nu_{s} \circ \Phi_{s}\right) J_{\Phi_{s}}\right]\right|_{s=0} d \mathcal{H}^{1}
\end{aligned}
$$


The first integral in the above expression can be written as

$$
\int_{\Gamma}\left(\nabla Q^{2} \cdot \dot{\Phi}_{0}\right) \dot{\Phi}_{0} \cdot \nu d \mathcal{H}^{1}=\int_{\Gamma}\left(\partial_{\tau} Q^{2}\left(\dot{\Phi}_{0} \cdot \tau\right)\left(\dot{\Phi}_{0} \cdot \nu\right)+\partial_{\nu} Q^{2}\left(\dot{\Phi}_{0} \cdot \nu\right)^{2}\right) d \mathcal{H}^{1} .
$$

Since $\nabla u=\partial_{\nu} u \nu$ on $\Gamma$, the first term in the second line of (2.18) becomes

$$
\begin{aligned}
-\int_{\Gamma} 2\left(\nabla u \cdot \nabla \dot{u}_{0}\right) \dot{\Phi}_{0} \cdot \nu d \mathcal{H}^{1} & =-\int_{\Gamma} 2 \partial_{\nu} \dot{u}_{0} \partial_{\nu} u \dot{\Phi}_{0} \cdot \nu d \mathcal{H}^{1} \\
& =\int_{\Gamma} 2 \dot{u}_{0} \partial_{\nu} \dot{u}_{0} d \mathcal{H}^{1}
\end{aligned}
$$

where we used the fact that $\dot{u}_{0}=-\left(X_{0} \cdot \nu\right) \partial_{\nu} u$ by (2.10) and $X_{0}=\dot{\Phi}_{0}$. We now focus on the term

$$
-\int_{\Gamma} 2\left(D^{2} u \nabla u \cdot \dot{\Phi}_{0}\right) \dot{\Phi}_{0} \cdot \nu d \mathcal{H}^{1} .
$$

Using again the fact that $\nabla u \cdot \tau=0$ on $\Gamma$ and that $u$ is harmonic, we obtain

$$
0=\partial_{\tau}(\nabla u \cdot \tau)=D^{2} u \tau \cdot \tau+\nabla u \cdot \partial_{\tau} \tau=-D^{2} u \nu \cdot \nu-\kappa \partial_{\nu} u \quad \text { on } \Gamma,
$$

that is,

$$
D^{2} u \nu \cdot \nu=-\kappa \partial_{\nu} u \quad \text { on } \Gamma .
$$

Thus,

$$
\begin{aligned}
& -\int_{\Gamma} 2\left(D^{2} u \nabla u \cdot \dot{\Phi}_{0}\right) \dot{\Phi}_{0} \cdot \nu d \mathcal{H}^{1} \\
& =-\int_{\Gamma} 2\left(D^{2} u \nabla u \cdot \tau\right)\left(\dot{\Phi}_{0} \cdot \tau\right)\left(\dot{\Phi}_{0} \cdot \nu\right) d \mathcal{H}^{1}-\int_{\Gamma} 2 \partial_{\nu} u\left(D^{2} u \nu \cdot \nu\right)\left(\dot{\Phi}_{0} \cdot \nu\right)^{2} d \mathcal{H}^{1} \\
& =-\int_{\Gamma} \partial_{\tau}\left(|\nabla u|^{2}\right)\left(\dot{\Phi}_{0} \cdot \tau\right)\left(\dot{\Phi}_{0} \cdot \nu\right) d \mathcal{H}^{1}+\int_{\Gamma} 2 \kappa\left(\partial_{\nu} u\right)^{2}\left(\dot{\Phi}_{0} \cdot \nu\right)^{2} d \mathcal{H}^{1}
\end{aligned}
$$

Finally, by [CMM, Lemma 3.8] we have

$$
\left.\frac{d}{d s}\left[\dot{\Phi}_{s} \cdot\left(\nu_{s} \circ \Phi_{s}\right) J_{\Phi_{s}}\right]\right|_{s=0}=\ddot{\Phi}_{0} \cdot \nu-2\left(\dot{\Phi}_{0} \cdot \tau\right) \partial_{\tau}\left(\dot{\Phi}_{0} \cdot \nu\right)+\kappa\left(\dot{\Phi}_{0} \cdot \tau\right)^{2}+\partial_{\tau}\left[\left(\dot{\Phi}_{0} \cdot \nu\right) \dot{\Phi}_{0}\right] \cdot \tau .
$$

Combining the previous equalities, we deduce that

$$
\begin{aligned}
\left.\frac{d^{2}}{d s^{2}} \mathcal{F}\left(u_{s}\right)\right|_{s=0}= & \int_{\Gamma} 2 \dot{u}_{0} \partial_{\nu} \dot{u}_{0} d \mathcal{H}^{1}+\int_{\Gamma}\left(\partial_{\nu} Q^{2}+2 \kappa\left(\partial_{\nu} u\right)^{2}\right)\left(\dot{\Phi}_{0} \cdot \nu\right)^{2} d \mathcal{H}^{1} \\
& +\int_{\Gamma}\left(Q^{2}-|\nabla u|^{2}\right)\left(\ddot{\Phi}_{0} \cdot \nu-2\left(\dot{\Phi}_{0} \cdot \tau\right) \partial_{\tau}\left(\dot{\Phi}_{0} \cdot \nu\right)+\kappa\left(\dot{\Phi}_{0} \cdot \tau\right)^{2}\right) d \mathcal{H}^{1} \\
& +\int_{\Gamma}\left(Q^{2}-|\nabla u|^{2}\right) \partial_{\tau}\left[\left(\dot{\Phi}_{0} \cdot \nu\right) \dot{\Phi}_{0}\right] \cdot \tau d \mathcal{H}^{1} \\
& +\int_{\Gamma} \partial_{\tau}\left(Q^{2}-|\nabla u|^{2}\right)\left(\dot{\Phi}_{0} \cdot \tau\right)\left(\dot{\Phi}_{0} \cdot \nu\right) d \mathcal{H}^{1}
\end{aligned}
$$


Now, using the fundamental theorem of calculus on curves, the last two integrals in the formula above satisfy

$$
\begin{aligned}
\int_{\Gamma}\left(Q^{2}-|\nabla u|^{2}\right) & \partial_{\tau}\left[\left(\dot{\Phi}_{0} \cdot \nu\right) \dot{\Phi}_{0}\right] \cdot \tau d \mathcal{H}^{1}+\int_{\Gamma} \partial_{\tau}\left(Q^{2}-|\nabla u|^{2}\right)\left(\dot{\Phi}_{0} \cdot \tau\right)\left(\dot{\Phi}_{0} \cdot \nu\right) d \mathcal{H}^{1} \\
& =\int_{\Gamma} \partial_{\tau}\left[\left(Q^{2}-|\nabla u|^{2}\right)\left(\dot{\Phi}_{0} \cdot \nu\right) \dot{\Phi}_{0}\right] \cdot \tau d \mathcal{H}^{1} \\
& =\int_{\Gamma} \kappa\left(Q^{2}-|\nabla u|^{2}\right)\left(\dot{\Phi}_{0} \cdot \nu\right)^{2} d \mathcal{H}^{1}
\end{aligned}
$$

Thus, we conclude that

$$
\begin{aligned}
\left.\frac{d^{2}}{d s^{2}} \mathcal{F}\left(u_{s}\right)\right|_{s=0}=\int_{\Gamma} 2 \dot{u}_{0} \partial_{\nu} \dot{u}_{0} d \mathcal{H}^{1}+\int_{\Gamma}\left(\partial_{\nu} Q^{2}+2 \kappa\left(\partial_{\nu} u\right)^{2}\right)\left(X_{0} \cdot \nu\right)^{2} d \mathcal{H}^{1} \\
+\int_{\Gamma}\left(Q^{2}-|\nabla u|^{2}\right)\left(Z_{0} \cdot \nu-2\left(X_{0} \cdot \tau\right) \partial_{\tau}\left(X_{0} \cdot \nu\right)+\kappa\left|X_{0}\right|^{2}\right) d \mathcal{H}^{1}
\end{aligned}
$$

where we used the fact that $\dot{\Phi}_{0}=X_{0}$ and $\ddot{\Phi}_{0}=Z_{0}$.

Let us now fix $r \in(0,1)$. We observe that the family of diffeomorphisms $\left\{\tilde{\Phi}_{h}\right\}_{h \in[0,1]}$ defined as

$$
\tilde{\Phi}_{h}:=\Phi_{r+h} \circ \Phi_{r}^{-1}
$$

is still an admissible flow (we can always reparametrize the variable $h$ away from 0 so that $\tilde{\Phi}_{h}$ is defined for all $\left.h \in[0,1]\right)$, and that

$$
\dot{\tilde{\Phi}}_{0}=X_{r}, \quad \ddot{\tilde{\Phi}}_{0}=Z_{r} .
$$

Applying (2.19), we deduce that

$$
\begin{aligned}
& \left.\frac{d^{2}}{d s^{2}} \mathcal{F}\left(u_{s}\right)\right|_{s=r}=\left.\frac{d^{2}}{d h^{2}} \mathcal{F}\left(u_{r+h}\right)\right|_{h=0} \\
& =\int_{\Gamma_{r}} 2 \dot{u}_{r} \partial_{\nu_{r}} \dot{u}_{r} d \mathcal{H}^{1}+\int_{\Gamma_{r}}\left(\partial_{\nu_{r}} Q^{2}+2 \kappa_{r}\left(\partial_{\nu_{r}} u_{r}\right)^{2}\right)\left(X_{r} \cdot \nu_{r}\right)^{2} d \mathcal{H}^{1} \\
& \quad+\int_{\Gamma_{r}}\left(Q^{2}-\left|\nabla u_{r}\right|^{2}\right)\left(Z_{r} \cdot \nu_{r}-2\left(X_{r} \cdot \tau_{r}\right) \partial_{\tau_{r}}\left(X_{r} \cdot \nu_{r}\right)+\kappa_{r}\left|X_{r}\right|^{2}\right) d \mathcal{H}^{1} .
\end{aligned}
$$

To conclude the proof of (2.13), it remains to show that

$$
\int_{\Gamma_{s}} 2 \dot{u}_{s} \partial_{\nu_{s}} \dot{u}_{s} d \mathcal{H}^{1}=\int_{\Phi_{s}\left(\Omega_{+}\right)} 2\left|\nabla \dot{u}_{s}\right|^{2} d \boldsymbol{x}
$$

Indeed, by (2.6) and the divergence theorem

$$
\begin{aligned}
\int_{\Gamma_{s}} 2 \dot{u}_{s} \partial_{\nu_{s}} \dot{u}_{s} d \mathcal{H}^{1} & =\int_{\partial \Phi_{s}\left(\Omega_{+}\right)} 2 \dot{u}_{s} \partial_{\nu_{s}} \dot{u}_{s} d \mathcal{H}^{1} \\
& =\int_{\Phi_{s}\left(\Omega_{+}\right)} 2\left(\dot{u}_{s} \Delta \dot{u}_{s}+\left|\nabla \dot{u}_{s}\right|^{2}\right) d \boldsymbol{x} \\
& =\int_{\Phi_{s}\left(\Omega_{+}\right)} 2\left|\nabla \dot{u}_{s}\right|^{2} d \boldsymbol{x} .
\end{aligned}
$$


Hence, 2.13) holds and the proof is complete.

\section{Construction of the Family $\Phi_{s}$}

Let $\Omega, u$, and $\Gamma$ be as in Section 2, and assume that

$$
\Gamma=\{(x, w(x)): x \in(-1,1)\},
$$

where $w$ is a periodic function with $w \in C^{3}(\mathbb{R})$ and

$$
w(x)>0 \text { for all } x \in[-1,1] .
$$

Let

$$
-1<a<b<1
$$

and consider a polynomial $\varphi:[a, b] \rightarrow \mathbb{R}$ satisfying

$$
\varphi(a)=\varphi^{\prime}(a)=\varphi^{\prime \prime}(a)=\varphi^{\prime \prime \prime}(a)=0, \quad \varphi(b)=\varphi^{\prime}(b)=\varphi^{\prime \prime}(b)=\varphi^{\prime \prime \prime}(b)=0,
$$

and such that $\|\varphi\|_{C^{2, \alpha}(a, b)}<<1$. Extend $\varphi$ to be zero outside $[a, b]$. In this section we construct an admissible flow (see Definition 2.1) joining $\Gamma$ to $\operatorname{graph}(w+\varphi)$. To estimate the second variation along the flow it is essential to have the condition $X_{s} \cdot \tau_{s}=0$ on $\Gamma_{s}$ for every $s$. This leads to a first order partial differential equation (see (3.41) below), that we solve using the method of characteristics. One of the main difficulties is that the components of the flow are given by compositions of functions that are discontinuous. Thus, proving the regularity of the flow is extremely delicate and it will be carried out in the appendix. The construction of the flow is the central part of this paper and will require several preliminary results.

Theorem 3.1 Let $\varphi$ and $w$ be as above. Then there exists an admissible flow $\left\{\Phi_{s}\right\}_{s \in[0,1]}$ such that

$$
\Phi_{s}(\Gamma)=\{(x, w(x)+s \varphi(x)): x \in(-1,1)\}
$$

and

$$
X_{s} \cdot \tau_{s}=0 \quad \text { on } \Gamma_{s}
$$

for every $s \in[0,1]$.

For every $x \in \mathbb{R}$, we consider the initial value problem

$$
\left\{\begin{array}{l}
\frac{d \xi}{d t}=-w^{\prime}(\xi) \varphi(\xi)-(\eta-w(\xi)) \varphi^{\prime}(\xi) \\
\frac{d \eta}{d t}=\varphi(\xi) \\
\xi(0)=x, \eta(0)=w(x)
\end{array}\right.
$$

Since $\varphi \in C_{c}^{3}(\mathbb{R}), w \in C^{3}(\mathbb{R})$, the function

$$
(\xi, \eta) \in \mathbb{R}^{2} \mapsto\left(-w^{\prime}(\xi) \varphi(\xi)-(\eta-w(\xi)) \varphi^{\prime}(\xi), \varphi(\xi)\right)
$$


is $C^{2}$, globally Lipschitz, and satisfies

$$
\left|\left(-w^{\prime}(\xi) \varphi(\xi)-(\eta-w(\xi)) \varphi^{\prime}(\xi), \varphi(\xi)\right)\right| \leq C(1+|\eta|)
$$

for all $(\xi, \eta) \in \mathbb{R}^{2}$ and for some constant $C>0$. Hence, the initial value problem (3.5) admits a unique global solution, which depends smoothly on the initial datum, and thus on $x$. We will denote by $(\xi(t, x), \eta(t, x)), t \in \mathbb{R}$, the solution of (3.5).

Observe that if $\varphi(x)=0$, then

$$
(\xi(t, x), \eta(t, x)) \equiv(x, w(x)) .
$$

Remark 3.2 Note that if $\varphi^{\prime}\left(x_{0}\right)=w^{\prime}\left(x_{0}\right)=0$ or $\varphi\left(x_{0}\right)=\varphi^{\prime}\left(x_{0}\right)=0$ for some $x_{0} \in \mathbb{R}$, then for every $y_{0} \in \mathbb{R}$ the unique solution of the initial value problem

$$
\left\{\begin{array}{l}
\frac{d \xi}{d t}=-w^{\prime}(\xi) \varphi(\xi)-(\eta-w(\xi)) \varphi^{\prime}(\xi), \\
\frac{d \eta}{d t}=\varphi(\xi) \\
\xi(0)=x_{0}, \eta(0)=y_{0}
\end{array}\right.
$$

is given by

$$
\xi\left(t, x_{0}\right) \equiv x_{0}, \quad \eta\left(t, x_{0}\right)=y_{0}+t \varphi\left(x_{0}\right) .
$$

Hence, if for some $\alpha<\beta$ we have $\varphi(\alpha)=\varphi^{\prime}(\alpha)=0$ and $\varphi(\beta)=\varphi^{\prime}(\beta)=0$, then for every $\alpha<x<\beta$ the curve $(\xi(\cdot, x), \eta(\cdot, x))$ cannot leave the vertical strip $(\alpha, \beta) \times \mathbb{R}$, otherwise uniqueness for the initial value problem (3.7) would be violated. In particular, in view of (3.2), if $a<x<b$ then the curve $(\xi(\cdot, x), \eta(\cdot, x))$ cannot leave the vertical strip $(a, b) \times \mathbb{R}$.

Theorem 3.3 Let $\varphi$ and $w$ be as above. Given $s \in[0,1]$ and $x \in \mathbb{R}$, there exists a first time $t_{0}=t_{0}(s, x) \geq 0$ such that the solution $(\xi(\cdot, x), \eta(\cdot, x))$ of (3.5) intersects the graph of the function $w+s \varphi$ at time $t_{0}$. Moreover, if $s=0$ or $\varphi(x)=0$ then

$$
t_{0}(s, x)=0,
$$

if $\varphi(x) \neq 0$ and $\varphi^{\prime}(x)=w^{\prime}(x)=0$ then

$$
t_{0}(s, x)=s,
$$

while in all the other cases

$$
0 \leq t_{0}(s, x) \leq s
$$

Finally, $t_{0}$ is of class $C^{2}$ in $[0,1] \times\{x \in \mathbb{R}: \varphi(x) \neq 0\}$ and if $\varphi(x) \neq 0$, then

$$
\varphi(\xi(t, x)) \varphi(x)>0 \quad \text { for all } 0 \leq t \leq t_{0}(s, x) .
$$

Proof. We begin by proving the existence of $t_{0}$. If $s=0$ or $\varphi(x)=0$, then $t_{0}(s, x)=0$ by (3.5). Property (3.10) follows from (3.7) and (3.8) with $x_{0}=x$ and $y_{0}=w(x)$. Thus, in what follows assume that $\varphi(x) \neq 0, s>0$, and that at least one of $\varphi^{\prime}(x)$ 
and $w^{\prime}(x)$ is different from zero. By (3.2) and Remark 3.2, the curve $(\xi(\cdot, x), \eta(\cdot, x))$ cannot leave the vertical strip $(a, b) \times \mathbb{R}$.

Step 1: Assume that $\varphi(x)>0$ and let $T>0$ be the first time, if it exists, such that $\varphi(\xi(T, x))=0$, otherwise set $T:=\infty$. Then $\varphi(\xi(t, x))>0$ for all $0 \leq t<T$, and so by (3.5), $\eta(\cdot, x)$ is strictly increasing in $[0, T)$ and there exists

$$
\lim _{t \rightarrow T^{-}} \eta(t, x)=\ell \in(w(x), \infty] .
$$

If $\ell=\infty$ (and hence $T=\infty$ ), then the solution $(\xi(\cdot, x), \eta(\cdot, x))$ of (3.5) intersects the graph of the function $w+s \varphi$, and so $t_{0}$ exists. Thus, in what follows it suffices to consider the case $\ell<\infty$. Assume, by contradiction, that

$$
\eta(t, x)<w(\xi(t, x))+s \varphi(\xi(t, x)) \quad \text { for all } 0<t \leq T .
$$

Substep 1a: We claim that the curve $(\xi(\cdot, x), \eta(\cdot, x))$ stays above the graph of the function $w$ for all $0<t \leq T$. Consider the function

$$
G(\xi, \eta):=\frac{\eta-w(\xi)}{\varphi(\xi)}
$$

defined for all $\varphi(\xi) \neq 0$ and $\eta \in \mathbb{R}$. Then

$$
\partial_{\xi} G(\xi, \eta)=\frac{-w^{\prime}(\xi) \varphi(\xi)-(\eta-w(\xi)) \varphi^{\prime}(\xi)}{\varphi^{2}(\xi)}, \quad \partial_{\eta} G(\xi, \eta)=\frac{1}{\varphi(\xi)},
$$

and so by (3.5), for all $0<t<T$,

$$
\begin{aligned}
& \partial_{t} \xi(t, x)=\varphi^{2}(\xi(t, x)) \partial_{\xi} G(\xi(t, x), \eta(t, x)), \\
& \partial_{t} \eta(t, x)=\varphi^{2}(\xi(t, x)) \partial_{\eta} G(\xi(t, x), \eta(t, x)) .
\end{aligned}
$$

It follows that for all $0<t<T$,

$$
\begin{aligned}
\frac{\left(\partial_{t} \xi(t, x)\right)^{2}+\left(\partial_{t} \eta(t, x)\right)^{2}}{\varphi^{2}(\xi(t, x))}= & \partial_{\xi} G(\xi(t, x), \eta(t, x)) \partial_{t} \xi(t, x) \\
& +\partial_{\eta} G(\xi(t, x), \eta(t, x)) \partial_{t} \eta(t, x) \\
= & \partial_{t}(G(\xi(t, x), \eta(t, x)) .
\end{aligned}
$$

Integrating between 0 and $t$, and using (3.5) once more, gives

$$
\int_{0}^{t} \frac{\left(\partial_{t} \xi(r, x)\right)^{2}+\left(\partial_{t} \eta(r, x)\right)^{2}}{\varphi^{2}(\xi(r, x))} d r=\frac{\eta(t, x)-w(\xi(t, x))}{\varphi(\xi(t, x))} .
$$

Since the integrand on the left-hand side is positive for all $0<t<T$, it follows that $\eta(t, x)>w(\xi(t, x))$ for all $0<t<T$. If $T<\infty$, then also by (3.14) we have that

$$
w(\xi(t, x))<\eta(t, x)<w(\xi(t, x))+s \varphi(\xi(t, x)),
$$


and letting $t \rightarrow T^{-}$gives that $w(\xi(T, x))=\eta(T, x)=w(\xi(T, x))+s \varphi(\xi(T, x))$, where we have used the fact that $\varphi(\xi(T, x))=0$. This contradicts (3.14), and thus establishes the existence of $t_{0}$ in the case $T<\infty$.

It remains to study the case $T=\infty$. Since $\frac{\left(\partial_{t} \eta(r, x)\right)^{2}}{\varphi^{2}(\xi(r, x))}=1$ by (3.5), it follows from (3.16) that

$$
t \leq \frac{\eta(t, x)-w(\xi(t, x))}{\varphi(\xi(t, x))}
$$

for all $t>0$. Let $\alpha<x<\beta$ be such that $\varphi>0$ in $(\alpha, \beta)$ and $\varphi(\alpha)=\varphi(\beta)=0$. We claim that there exists

$$
\lim _{t \rightarrow \infty} \xi(t, x)=l \in\{\alpha, \beta\}
$$

To see this, note that since $\varphi(\xi(t, x))>0$ for all $t$, we have that

$$
\alpha \leq l_{1}:=\liminf _{t \rightarrow \infty} \xi(t, x), \quad l_{2}:=\limsup _{t \rightarrow \infty} \xi(t, x) \leq \beta .
$$

Assume, by contradiction, that $l_{1}<l_{2}$. Then there exists a sequence $t_{i} \rightarrow \infty$ such that $\xi\left(t_{i}, x\right) \rightarrow c \in(\alpha, \beta)$. Taking $t=t_{i}$ in (3.17), and using (3.13) and the fact that $\ell<\infty$, gives

$$
\infty=\lim _{i \rightarrow \infty} t_{i}=\lim _{i \rightarrow \infty} \frac{\eta\left(t_{i}, x\right)-w\left(\xi\left(t_{i}, x\right)\right)}{\varphi\left(\xi\left(t_{i}, x\right)\right)}=\frac{\ell-w(c)}{\varphi(c)}<\infty,
$$

which is a contradiction. Hence, $l_{1}=l_{2}$. Note that the previous argument also shows that $l_{1}$ cannot belong to $(\alpha, \beta)$. Hence, either $l_{1}=\alpha$ or $l_{2}=\beta$.

Substep 1b: We prove the existence of $t_{0}$. Without loss of generality, assume that $l=\alpha$ (the case $l=\beta$ is similar). Then by (3.14) and Substep 1a, we have

$$
w(\xi(t, x))<\eta(t, x)<w(\xi(t, x))+s \varphi(\xi(t, x))
$$

for all $t>0$. Hence,

$$
0<\frac{\eta(t, x)-w(\xi(t, x))}{\varphi(\xi(t, x))}<s .
$$

Letting $t \rightarrow \infty$ we obtain a contradiction from (3.17). Therefore, we have proved that condition (3.14) fails. This asserts the existence of $t_{0}$.

Substep 1c: We prove (3.12). It follows from Substeps 1a and $1 \mathrm{~b}$ that $t_{0}(s, x) \leq T$, so that

$$
\varphi(\xi(t, x)) \varphi(x)>0 \text { for all } 0 \leq t<t_{0}(s, x) .
$$

To prove (3.12), it remains to show that $\varphi\left(\xi\left(t_{0}(s, x), x\right)\right)>0$. Let $t_{0}:=t_{0}(s, x)$. Assume, by contradiction, that $\varphi\left(\xi\left(t_{0}, x\right)\right)=0$. Then by the definition of $t_{0}(s, x)$ we have that

$$
\eta\left(t_{0}, x\right)=w\left(\xi\left(t_{0}, x\right)\right)
$$

which contradicts the fact that the unique solution of the initial value problem

$$
\left\{\begin{array}{l}
\frac{d \xi}{d t}=-w^{\prime}(\xi) \varphi(\xi)-(\eta-w(\xi)) \varphi^{\prime}(\xi), \\
\frac{d \eta}{d t}=\varphi(\xi) \\
\xi\left(t_{0}\right)=\xi\left(t_{0}, x\right), \eta\left(t_{0}\right)=w\left(\xi\left(t_{0}, x\right)\right)
\end{array}\right.
$$


is given by

$$
\xi_{1}(t) \equiv \xi\left(t_{0}, x\right), \quad \eta_{1}\left(t, x_{0}\right) \equiv w\left(\xi\left(t_{0}, x\right)\right) .
$$

Step 2: The case $\varphi(x)<0$ is similar and we omit it.

Step 3: We prove the regularity of $t_{0}$. Fix $\left(s_{0}, x_{0}\right) \in[0,1] \times \mathbb{R}$, with $\varphi\left(x_{0}\right) \neq 0$, and let $t_{0}:=t_{0}\left(s_{0}, x_{0}\right)$. Assume that $\varphi\left(x_{0}\right)>0$ (the case $\varphi\left(x_{0}\right)<0$ is similar), and let $\alpha<x_{0}<\beta$ be such that $\varphi>0$ in $(\alpha, \beta)$ and $\varphi(\alpha)=\varphi(\beta)=0$. Consider the $C^{2}$ function

$$
F(s, t, x):=\eta(t, x)-w(\xi(t, x))-s \varphi(\xi(t, x))
$$

defined in the set

$$
V:=\mathbb{R} \times \mathbb{R} \times(\alpha, \beta) .
$$

Then $F\left(s_{0}, t_{0}, x_{0}\right)=0$. By (3.5) and (3.12), we have

$$
\begin{aligned}
\partial_{t} F\left(s_{0}, t_{0}, x_{0}\right) & =\partial_{t} \eta\left(t_{0}, x_{0}\right)-\left[w^{\prime}\left(\xi\left(t_{0}, x_{0}\right)\right)+s_{0} \varphi^{\prime}\left(\xi\left(t_{0}, x_{0}\right)\right)\right] \partial_{t} \xi\left(t_{0}, x_{0}\right) \\
& =\varphi\left(\xi\left(t_{0}, x_{0}\right)\right)\left[1+\left(w^{\prime}\left(\xi\left(t_{0}, x_{0}\right)\right)+s_{0} \varphi^{\prime}\left(\xi\left(t_{0}, x_{0}\right)\right)\right)^{2}\right]>0 .
\end{aligned}
$$

Thus, we can apply the implicit function theorem to conclude that there exist $0<$ $r<\min \left\{\beta-x_{0}, x_{0}-\alpha\right\}, \delta>0$, a function $t_{1}: B\left(\left(s_{0}, x_{0}\right) ; r\right) \rightarrow\left[t_{0}-\delta, t_{0}+\delta\right]$ of class $C^{2}$ such that $t_{1}\left(s_{0}, x_{0}\right)=t_{0}$ and

$$
F\left(s, t_{1}(s, x), x\right)=0 \quad \text { for all }(s, x) \in B\left(\left(s_{0}, x_{0}\right) ; r\right) .
$$

Note that, in view of (3.16), which continues to hold for $t<0$ small, and the fact that $\varphi>0$, for $t<0$ sufficiently small,

$$
\eta(t, x)<w(\xi(t, x)) \leq w(\xi(t, x))+s \varphi(\xi(t, x)),
$$

and so the function $t_{1}$ must be nonnegative. Hence, by the definition of $t_{0}(s, x)$, we have

$$
t_{0}(s, x) \leq t_{1}(s, x) \text { for all }(s, x) \in B\left(\left(s_{0}, x_{0}\right) ; r\right) .
$$

We claim that $t_{1}(s, x)=t_{0}(s, x)$ for all $(s, x) \in B\left(\left(s_{0}, x_{0}\right) ; r_{1}\right)$ for some $0<r_{1}<r$. Since $t_{0}$ is the first time that the solution $\left(\xi\left(\cdot, x_{0}\right), \eta\left(\cdot, x_{0}\right)\right)$ of (3.5) intersects the graph of the function $w+s_{0} \varphi$, if $t_{0}>0$ we have that

$$
\eta\left(t, x_{0}\right)<w\left(\xi\left(t, x_{0}\right)\right)+s_{0} \varphi\left(\xi\left(t, x_{0}\right)\right) \quad \text { for all } 0 \leq t<t_{0} .
$$

Fix $0<\varepsilon<\delta$ and let

$$
c_{\varepsilon}:=\min _{0 \leq t \leq t_{0}-\varepsilon}\left(w\left(\xi\left(t, x_{0}\right)\right)+s_{0} \varphi\left(\xi\left(t, x_{0}\right)\right)-\eta\left(t, x_{0}\right)\right)>0 .
$$

By the regularity of $w$ and $\varphi$ and the continuity of $\xi$ and $\eta$ with respect to initial data, there exists $0<r_{1}<r$ such that

$$
\left|w(\xi(t, x))+s \varphi(\xi(t, x))-\eta(t, x)-\left(w\left(\xi\left(t, x_{0}\right)\right)+s_{0} \varphi\left(\xi\left(t, x_{0}\right)\right)-\eta\left(t, x_{0}\right)\right)\right| \leq \frac{1}{2} c_{\varepsilon}
$$


for all $(s, x) \in B\left(\left(s_{0}, x_{0}\right) ; r_{1}\right)$ and for all $t \in\left[0, t_{0}-\varepsilon\right]$. Hence,

$$
\eta(t, x)<w(\xi(t, x))+s \varphi(\xi(t, x)) \quad \text { for all } 0 \leq t<t_{0}-\varepsilon
$$

for all $(s, x) \in B\left(\left(s_{0}, x_{0}\right) ; r_{1}\right)$. This implies that

$$
t_{0}-\varepsilon \leq t_{0}(s, x)
$$

for all $(s, x) \in B\left(\left(s_{0}, x_{0}\right) ; r_{1}\right)$. If $t_{0}=0$, then (3.19) continues to hold since $t_{0}(s, x) \geq$ 0 . On the other hand, since $t_{1}$ is continuous and $t_{1}\left(s_{0}, x_{0}\right)=t_{0}$, by taking $r_{1}$ smaller if necessary, we have that $t_{0}-\varepsilon \leq t_{1}(s, x) \leq t_{0}+\varepsilon$ for all $(s, x) \in B\left(\left(s_{0}, x_{0}\right) ; r_{1}\right)$. Because $t_{0}(s, x) \leq t_{1}(s, x)$, also by (3.19), we have that

$$
t_{0}-\varepsilon \leq t_{0}(s, x) \leq t_{0}+\varepsilon
$$

for all $(s, x) \in B\left(\left(s_{0}, x_{0}\right) ; r_{1}\right)$. Using the fact that $\varepsilon<\delta$, it follows from the uniqueness of the implicit function that $t_{0}(s, x)=t_{1}(s, x)$ for all $(s, x) \in B\left(\left(s_{0}, x_{0}\right) ; r_{1}\right)$. In turn,

$$
F\left(s, t_{0}(s, x), x\right)=0 \quad \text { for all }(s, x) \in B\left(\left(s_{0}, x_{0}\right) ; r_{1}\right),
$$

and so, by (3.5) and the definition of $t_{0}$, we have

$$
\begin{aligned}
\partial_{x} t_{0}(s, x) & =-\frac{\partial_{x} F\left(s, t_{0}(s, x), x\right)}{\partial_{t} F\left(s, t_{0}(s, x), x\right)} \\
& =\frac{\left[w^{\prime}\left(\xi\left(t_{0}(s, x), x\right)\right)+s \varphi^{\prime}\left(\xi\left(t_{0}(s, x), x\right)\right)\right] \partial_{x} \xi\left(t_{0}(s, x), x\right)-\partial_{x} \eta\left(t_{0}(s, x), x\right)}{\varphi\left(\xi\left(t_{0}(s, x), x\right)\right)\left[1+\left(w^{\prime}\left(\xi\left(t_{0}(s, x), x\right)\right)+s \varphi^{\prime}\left(\xi\left(t_{0}(s, x), x\right)\right)\right)^{2}\right]} .
\end{aligned}
$$

Step 4: It remains to prove (3.11). By (3.17) and the definition of $t_{0}$, we have that

$$
0 \leq t_{0}(s, x) \leq \frac{\eta\left(t_{0}(s, x), x\right)-w\left(\xi\left(t_{0}(s, x), x\right)\right)}{\varphi\left(\xi\left(t_{0}(s, x), x\right)\right)}=s .
$$

This concludes the proof.

Remark 3.4 By (3.5), if $x \in \mathbb{R}$ and $0 \leq t \leq 1$, then

$$
|\eta(t, x)-w(x)|=\left|\int_{0}^{t} \varphi(\xi(r, x)) d r\right| \leq\|\varphi\|_{C([a, b])}
$$

and, in turn,

$$
\begin{aligned}
|\xi(t, x)-x| & =\left|\int_{0}^{t}\left[-w^{\prime}(\xi(r, x)) \varphi(\xi(r, x))-(\eta(r, x)-w(\xi(r, x))) \varphi^{\prime}(\xi(r, x))\right] d r\right| \\
& \leq 3\|w\|_{C^{1}([a, b])}\|\varphi\|_{C^{1}([a, b])}+\|\varphi\|_{C([a, b])}\left\|\varphi^{\prime}\right\|_{C([a, b])} .
\end{aligned}
$$

Since $0 \leq t_{0}(s, x) \leq s \leq 1$ by Theorem [3.3. it follows that

$$
\begin{gathered}
|\xi(t, x)-x| \leq 3\left(\|w\|_{C^{1}([a, b])}+\|\varphi\|_{C([a, b])}\right)\|\varphi\|_{C^{1}([a, b])}, \\
|\eta(t, x)-w(x)| \leq\|\varphi\|_{C([a, b])}
\end{gathered}
$$

for all $s \in[0,1], x \in \mathbb{R}$, and $0 \leq t \leq t_{0}(s, x)$. 
Given $s \in[0,1]$ and $x \in \mathbb{R}$, we define the function $g:[0,1] \times \mathbb{R} \rightarrow \mathbb{R}$ by

$$
g(s, x):=\xi\left(t_{0}(s, x), x\right),
$$

where $t_{0}(s, x)$ is given by Theorem 3.3 Note that by the definition of $t_{0}(s, x)$, if $s=0$ or $\varphi(x)=0$, then $t_{0}(s, x)=0$, and so

$$
g(s, x)=\xi(0, x)=x .
$$

Moreover, since $\left(\xi\left(t_{0}(s, x), x\right), \eta\left(t_{0}(s, x), x\right)\right)$ belongs to the graph of $w+s \varphi$, we have that

$$
\begin{aligned}
\eta\left(t_{0}(s, x), x\right) & =w\left(\xi\left(t_{0}(s, x), x\right)\right)+s \varphi\left(\xi\left(t_{0}(s, x), x\right)\right) \\
& =w(g(s, x))+s \varphi(g(s, x))
\end{aligned}
$$

We will use this property in the sequel.

The following theorem states that the function $g$ is of class $C^{2}$. As part of the proof we will actually show that the function $t_{0}$ is discontinuous at all points $\left(s, x_{0}\right)$ with $\varphi\left(x_{0}\right)=0$ and $\varphi \neq 0$ near $x_{0}$. Hence, establishing the regularity of $g$ is far from trivial. The proof of Theorem 3.5 is rather lengthy and will be presented in the appendix.

Theorem 3.5 Let $\varphi$ and $w$ be as above. Then the function $g:[0,1] \times \mathbb{R} \rightarrow \mathbb{R}$ defined in (3.22) is of class $C^{2}$.

Define the function $h:[0,1] \times \mathbb{R} \rightarrow \mathbb{R}$ by

$$
h(s, x):=\eta\left(t_{0}(s, x), x\right),
$$

where $t_{0}(s, x)$ is given by Theorem 3.3 Note that, by (3.24),

$$
h(s, x)=w(g(s, x))+s \varphi(g(s, x)) .
$$

Thus in view of Theorem 3.5, the function $h$ is of class $C^{2}$. Moreover, by (3.23), if $s=0$ or $\varphi(x)=0$, then

$$
h(s, x)=w(x) .
$$

Theorem 3.6 Let $\varphi$ and $w$ be as above. Then for every $s \in[0,1]$,

$$
\{(g(s, x), h(s, x)): x \in[a, b]\}=\{(x, w(x)+s \varphi(x)): x \in[a, b]\} .
$$

Proof. Given $s_{0} \in[0,1]$ and $x_{0} \in[a, b]$, we want to find $x \in[a, b]$ such that

$$
\left(\xi\left(t_{0}\left(s_{0}, x\right), x\right), \eta\left(t_{0}\left(s_{0}, x\right), x\right)\right)=\left(x_{0}, w\left(x_{0}\right)+s_{0} \varphi\left(x_{0}\right)\right) .
$$

If $s_{0}=0$ or $\varphi\left(x_{0}\right)=0$, then by (3.23) and (3.27), $g\left(s_{0}, x_{0}\right)=x_{0}$ and $h\left(s_{0}, x_{0}\right)=w\left(x_{0}\right)$ and so there is nothing to prove. Therefore, also by (3.2), in what follows we assume that $s_{0}>0, x_{0} \in(a, b)$, and $\varphi\left(x_{0}\right) \neq 0$. Assume further that $\varphi\left(x_{0}\right)>0$ (the case $\varphi\left(x_{0}\right)<0$ is similar). 
Consider the initial value problem

$$
\left\{\begin{array}{l}
\frac{d \xi}{d t}=w^{\prime}(\xi) \varphi(\xi)+(\eta-w(\xi)) \varphi^{\prime}(\xi), \\
\frac{d \eta}{d t}=-\varphi(\xi) \\
\xi(0)=x_{0}, \eta(0)=w\left(x_{0}\right)+s_{0} \varphi\left(x_{0}\right) .
\end{array}\right.
$$

Reasoning as for (3.5), we have that (3.28) admits a unique solution $\left(\xi_{0}, \eta_{0}\right)$ defined for all $t \in \mathbb{R}$. We claim that $\left(\xi_{0}, \eta_{0}\right)$ intersects the graph of $w$ at some time $t_{1}>0$.

For every $y \in \mathbb{R}$ the functions

$$
\begin{array}{ll}
\xi_{1}(t) \equiv a, & \eta_{1}(t) \equiv y \\
\xi_{2}(t) \equiv b, & \eta_{2}(t) \equiv y
\end{array}
$$

are solutions of the differential system in (3.28) with $\xi(0)=a, \eta(0)=y$, and $\xi(0)=b$, $\eta(0)=y$, respectively. Hence, by uniqueness of (3.28), we conclude that the curve $\left(\xi_{0}, \eta_{0}\right)$ cannot leave the vertical strip $(a, b) \times \mathbb{R}$.

Let $T>0$ be the first time, if it exists, such that $\varphi\left(\xi_{0}(T)\right)=0$, otherwise set $T:=\infty$. Then by (3.28) the function $\eta_{0}$ is strictly decreasing in $[0, T)$, and so there exists

$$
\lim _{t \rightarrow T} \eta_{0}(t)=l_{2} \in\left[-\infty, w\left(x_{0}\right)+s_{0} \varphi\left(x_{0}\right)\right) .
$$

If $l_{2}=-\infty$ (and hence $\left.T=\infty\right)$, then there exists a time $t_{1}>0$ such that $\left(\xi_{0}, \eta_{0}\right)$ intersects the graph of $w$. Thus, assume that $l_{2} \in \mathbb{R}$ and that

$$
w\left(\xi_{0}(t)\right)<\eta_{0}(t) \text { for all } 0 \leq t \leq T .
$$

Reasoning as in Substep 1a of the proof of Theorem 3.3 with $G$ in (3.15) replaced by

$$
G(\xi, \eta):=\frac{w(\xi)-\eta}{\varphi(\xi)},
$$

we have that

$$
\begin{aligned}
\int_{0}^{t} \frac{\left(\partial_{t} \xi_{0}(r)\right)^{2}+\left(\partial_{t} \eta_{0}(r)\right)^{2}}{\varphi^{2}\left(\xi_{0}(r)\right)} d r & =G\left(\xi_{0}(t), \eta_{0}(t)\right)-G\left(x_{0}, w\left(x_{0}\right)+s_{0} \varphi\left(x_{0}\right)\right) \\
& =\frac{w\left(\xi_{0}(t)\right)-\eta_{0}(t)}{\varphi\left(\xi_{0}(t)\right)}+s_{0}
\end{aligned}
$$

for all $0 \leq t<T$. Since the integrand on the left-hand side is positive for $t>0$, it follows that $\eta_{0}(t)<w\left(\xi_{0}(t)\right)+s_{0} \varphi\left(\xi_{0}(t)\right)$ for all $0<t<T$. As in Substep 1a of the proof of Theorem 3.3. if $T<\infty$ then we obtain a contradiction to (3.29). Thus, we can assume that $T=\infty$. As in Subtep 1a of the proof of Theorem 3.3. the inequality

$$
t \leq \frac{w\left(\xi_{0}(t)\right)-\eta_{0}(t)}{\varphi\left(\xi_{0}(t)\right)}+s_{0}
$$


for all $t>0$ implies (3.18). The existence of $t_{1}$ follows exactly as in Substep 1b of the proof of Theorem 3.3

This shows that $\left(\xi_{0}, \eta_{0}\right)$ intersects the graph of $w$ at some time $t_{1}>0$. Assume that $t_{1}$ is the first such time. Define $x:=\xi_{0}\left(t_{1}\right)$. Then the function $\left(\xi_{0}\left(t_{1}-\cdot\right), \eta_{0}\left(t_{1}-\cdot\right)\right)$ is the solution $(\xi(\cdot, x), \eta(\cdot, x))$ of the initial value problem (3.5), and at time $t=t_{1}$ it touches the graph of $w+s_{0} \varphi$ at the point $\left(x_{0}, w\left(x_{0}\right)+s_{0} \varphi\left(x_{0}\right)\right)$. Hence, $t_{0}\left(s_{0}, x\right)=t_{1}$ and

$$
\left(\xi\left(t_{0}(s, x), x\right), \eta\left(t_{0}(s, x), x\right)\right)=\left(x_{0}, w\left(x_{0}\right)+s_{0} \varphi\left(x_{0}\right)\right) .
$$

This completes the proof.

To estimate the norm of $\partial_{x} g$ and $\partial_{x} h$ we need the following preliminary result.

Proposition 3.7 Let $\varphi$ and $w$ be as above with $\|\varphi\|_{C^{2}([a, b])}<1$. Then

$$
\left|\partial_{x} \xi(t, x)-1\right| \leq C\|\varphi\|_{C^{2}([a, b])}, \quad\left|\partial_{x} \eta(t, x)-w^{\prime}(x)\right| \leq C\|\varphi\|_{C^{2}([a, b])}
$$

for all $x \in \mathbb{R}$ and $0 \leq t \leq t_{0}(s, x)$, where $C>0$ depends on $\|w\|_{C^{2}([a, b])}$.

Proof. Differentiating (3.5) with respect to $x$, we have that

$$
\partial_{t}\left(\left|\partial_{x} \xi\right|^{2}+\left|\partial_{x} \eta\right|^{2}\right)=-2\left[w^{\prime \prime}(\xi) \varphi(\xi)+(\eta-w(\xi)) \varphi^{\prime \prime}(\xi)\right]\left|\partial_{x} \xi\right|^{2} .
$$

If $\varphi(x)=0$, then by (3.6) the right-hand side of the previous equality is identically equal to zero. If $\varphi(x) \neq 0$ then assume that $\varphi(x)>0$ (the case $\varphi(x)<0$ is similar). Using the fact that

$$
w(\xi(t, x)) \leq \eta(t, x) \leq w(\xi(t, x))+s \varphi(\xi(t, x))
$$

for all $0 \leq t \leq t_{0}(s, x)$ (see Step 1 of the proof of Theorem 3.3), we obtain

$$
\begin{aligned}
& \left|\partial_{x} \xi(t, x)\right|^{2}+\left|\partial_{x} \eta(t, x)\right|^{2} \leq 1+\left(w^{\prime}(x)\right)^{2} \\
& \quad+2\|\varphi\|_{C([a, b])}\left(\left\|w^{\prime \prime}\right\|_{C([a, b])}+\left\|\varphi^{\prime \prime}\right\|_{C([a, b])}\right) \int_{0}^{t}\left(\left|\partial_{x} \xi(r, x)\right|^{2}+\left|\partial_{x} \eta(r, x)\right|^{2}\right) d r
\end{aligned}
$$

for all $0 \leq t \leq t_{0}(s, x)$. By Gronwall's inequality and the facts that $t_{0} \leq 1$ by Theorem 3.3 and $\|\varphi\|_{C^{2}([a, b])}<1$, we deduce that

$$
\begin{aligned}
& \left|\partial_{x} \xi(t, x)\right|^{2}+\left|\partial_{x} \eta(t, x)\right|^{2} \\
& \quad \leq\left(1+\left\|w^{\prime}\right\|_{C([a, b])}^{2}\right) \exp \left(2\left\|w^{\prime \prime}\right\|_{C([a, b])}+2\right)
\end{aligned}
$$

for $0 \leq t \leq t_{0}(s, x)$. In turn,

$$
\begin{aligned}
& \left|\partial_{x} \eta(t, x)-w^{\prime}(x)\right| \leq \int_{0}^{t}\left|\varphi^{\prime}(\xi(r, x)) \partial_{x} \xi(r, x)\right| d r \\
& \quad \leq\left\|\varphi^{\prime}\right\|_{C([a, b])}\left(1+\left\|w^{\prime}\right\|_{C([a, b])}\right) \exp \left(\left\|w^{\prime \prime}\right\|_{C([a, b])}+1\right) .
\end{aligned}
$$


This implies that

$$
\begin{aligned}
& \left|\partial_{x} \xi(t, x)-1\right| \\
& \leq \int_{0}^{t} \mid\left[w^{\prime \prime}(\xi(r, x)) \varphi(\xi(r, x))+(\eta(r, x)-w(\xi(r, x))) \varphi^{\prime \prime}(\xi(r, x))\right] \partial_{x} \xi(r, x) \\
& \quad+\varphi^{\prime}(\xi(r, x)) \partial_{x} \eta(r, x) \mid d r \\
& \leq\|\varphi\|_{C([a, b])}\left(\left\|w^{\prime \prime}\right\|_{C([a, b])}+\left\|\varphi^{\prime \prime}\right\|_{C([a, b])}\right) \\
& \quad \times\left(1+\left\|w^{\prime}\right\|_{C([a, b])}\right) \exp \left(\left\|w^{\prime \prime}\right\|_{C([a, b])}+1\right) \\
& \quad+\left\|\varphi^{\prime}\right\|_{C([a, b])}\left(1+\left\|w^{\prime}\right\|_{C([a, b])}\right) \exp \left(\left\|w^{\prime \prime}\right\|_{C([a, b])}+1\right) .
\end{aligned}
$$

Since $\|\varphi\|_{C^{2}([a, b])}<1$, this concludes the proof.

Theorem 3.8 Let $\varphi$ and $w$ be as above with $\|\varphi\|_{C^{2}([a, b])}<1$. Then

$$
\begin{aligned}
& \left|\partial_{x} g(s, x)-1\right| \leq C_{0}\|\varphi\|_{C^{2}([a, b])}, \\
& \left|\partial_{x} h(s, x)-w^{\prime}(x)\right| \leq C_{0}\|\varphi\|_{C^{2}([a, b])}
\end{aligned}
$$

for all $(s, x) \in[0,1] \times \mathbb{R}$, where $C_{0}>0$ depends on $\|w\|_{C^{2}([a, b])}$.

Proof. The proof is subdivided into three steps.

Step 1: By (5.9) in the Appendix, for $s \geq 0$ and $\varphi(x)>0$ (the case $\varphi(x)<0$ is similar), we have

$$
\begin{aligned}
\partial_{x} g(s, x)-1=\frac{\partial_{x} \xi\left(t_{0}(s, x), x\right)-1}{1+\left[w^{\prime}(g(s, x))+s \varphi^{\prime}(g(s, x))\right]^{2}} \\
+\frac{\left[w^{\prime}(g(s, x))+s \varphi^{\prime}(g(s, x))\right]\left[w^{\prime}(x)-w^{\prime}(g(s, x))-s \varphi^{\prime}(g(s, x))\right]}{1+\left[w^{\prime}(g(s, x))+s \varphi^{\prime}(g(s, x))\right]^{2}} \\
+\frac{w^{\prime}(g(s, x))+s \varphi^{\prime}(g(s, x))}{1+\left[w^{\prime}(g(s, x))+s \varphi^{\prime}(g(s, x))\right]^{2}}\left[\partial_{x} \eta\left(t_{0}(s, x), x\right)-w^{\prime}(x)\right] .
\end{aligned}
$$

By the mean value theorem, (3.5), (3.22), the facts that $t_{0}(s, x) \leq 1$ and $w(\xi) \leq \eta \leq$ $w(\xi)+s \varphi(\xi)$ (see Theorem 3.3), we obtain

$$
\begin{aligned}
\left|w^{\prime}(x)-w^{\prime}(g(s, x))\right|= & \left|w^{\prime \prime}(c)(x-g(s, x))\right| \\
\leq & \left\|w^{\prime \prime}\right\|_{C([a, b])} \int_{0}^{t_{0}(s, x)} \mid w^{\prime}(\xi(r, x)) \varphi(\xi(r, x)) \\
& +(\eta(r, x)-w(\xi(r, x))) \varphi^{\prime}(\xi(r, x)) \mid d r \\
\leq & \left\|w^{\prime \prime}\right\|_{C([a, b])}\left(\left\|w^{\prime}\right\|_{C([a, b])}+\left\|\varphi^{\prime}\right\|_{C([a, b])}\right)\|\varphi\|_{C([a, b])} .
\end{aligned}
$$

Hence, from (3.33) and Proposition 3.7, we deduce that

$$
\begin{aligned}
\left|\partial_{x} g(s, x)-1\right| \leq & \left|\partial_{x} \xi\left(t_{0}(s, x), x\right)-1\right|+\left|w^{\prime}(x)-w^{\prime}(g(s, x))\right|+\left|\varphi^{\prime}(g(s, x))\right| \\
& +\left|\partial_{x} \eta\left(t_{0}(s, x), x\right)-w^{\prime}(x)\right| \leq C\|\varphi\|_{C^{2}([a, b])} .
\end{aligned}
$$


Step 2: If $s \geq 0$ and $\varphi(x)=0$, then by (5.23) in the Appendix,

$$
\begin{aligned}
& \left|\partial_{x} g(s, x)-1\right|=1-\frac{\sqrt{1+\left[w^{\prime}(x)\right]^{2}}}{\sqrt{1+\left[w^{\prime}(x)+s \varphi^{\prime}(x)\right]^{2}}} \\
& \quad=\frac{\left[w^{\prime}(x)+s \varphi^{\prime}(x)\right]^{2}-\left[w^{\prime}(x)\right]^{2}}{\left(\sqrt{1+\left[w^{\prime}(x)\right]^{2}}+\sqrt{1+\left[w^{\prime}(x)+s \varphi^{\prime}(x)\right]^{2}}\right) \sqrt{1+\left[w^{\prime}(x)+s \varphi^{\prime}(x)\right]^{2}}} \\
& \quad \leq 2\left\|w^{\prime}\right\|_{C([a, b])}\left\|\varphi^{\prime}\right\|_{C([a, b])}+\left\|\varphi^{\prime}\right\|_{C([a, b])}^{2},
\end{aligned}
$$

so that 3.31) holds even in this case.

Step 3: To conclude the proof, note that by (3.26),

$$
\partial_{x} h(s, x)=\left[w^{\prime}(g(s, x))+s \varphi^{\prime}(g(s, x))\right] \partial_{x} g(s, x),
$$

and so by (3.31) and (3.34), we deduce that

$$
\begin{aligned}
\left|\partial_{x} h(s, x)-w^{\prime}(x)\right| \leq & \left|w^{\prime}(g(s, x))+s \varphi^{\prime}(g(s, x))\right|\left|\partial_{x} g(s, x)-1\right| \\
& +\left|w^{\prime}(g(s, x))-w^{\prime}(x)\right|+\left|\varphi^{\prime}(g(s, x))\right| \leq C\|\varphi\|_{C^{2}([a, b])},
\end{aligned}
$$

which proves (3.32).

We are now ready to construct the family of diffeomorphisms.

Proof of Theorem 3.1, For every $s \in[0,1]$, we define $\Psi_{s}: \mathbb{R}^{2} \rightarrow \mathbb{R}^{2}$ by

$$
\Psi_{s}(x, y):=(g(s, x), h(s, x)+y-w(x)),
$$

where $g$ and $h$ are the functions given in (3.22) and (3.25), respectively. By Theorem 3.5 and (3.26), $\Psi_{s}$ is of class $C^{2}\left([0,1] \times \mathbb{R}^{2}\right)$. Moreover, by (3.23) and (3.27),

$$
\Psi_{0}(x, y)=(x, y)
$$

which implies, in particular, that $\Psi_{0}(\Gamma)=\Gamma$, while by Theorem 3.6 and the fact that $g(s, x)=x$ and $h(s, x)=w(x)$ for all $x \in \mathbb{R} \backslash[a, b]$ by (3.23) and (3.27), it follows that

$$
\Psi_{s}(\Gamma)=\left\{\Psi_{s}(x, w(x)): x \in(-1,1)\right\}=\{(x, w(x)+s \varphi(x)): x \in(-1,1)\}
$$

for every $s \in[0,1]$.

Since $\min _{[-1,1]} w>0$ by (3.1), let

$$
0<2 L<\min _{[-1,1]} w, \quad M>\max _{[-1,1]} w .
$$

We now modify $\Psi_{s}$ to obtain a diffeomorphism in $\mathbb{R}^{2}$ which coincides with the identity outside the open set $U:=(a, b) \times(L, M+2)$. Given

$$
0<\delta_{0}<\min \{1, L\},
$$


construct a function $\lambda \in C_{c}^{\infty}(\mathbb{R})$ such that $0 \leq \lambda \leq 1, \lambda(y)=1$ if $L+\delta_{0} \leq y \leq$ $M+2-\delta_{0}, \lambda(y)=0$ if $y \geq M+2$ or $y \leq L$, and $\left|\lambda^{\prime}(y)\right| \leq 2 / \delta_{0}$ for all $y \in \mathbb{R}$. For every $s \in[0,1]$ and $(x, y) \in \mathbb{R}^{2}$, define

$$
\Phi_{s}(x, y):=\lambda(y) \Psi_{s}(x, y)+(1-\lambda(y))(x, y) .
$$

Then

$$
D \Phi_{s}(x, y)=I_{2 \times 2}+\lambda(y)\left(D \Psi_{s}(x, y)-I_{2 \times 2}\right)+\left(\Psi_{s}(x, y)-(x, y)\right) \otimes\left(0, \lambda^{\prime}(y)\right),
$$

and so

$$
\left|D \Phi_{s}(x, y)-I_{2 \times 2}\right| \leq\left|D \Psi_{s}(x, y)-I_{2 \times 2}\right|+\frac{2}{\delta_{0}}\left|\Psi_{s}(x, y)-(x, y)\right| .
$$

By Theorem 3.8 and (3.35), we have

$$
\left|D \Psi_{s}(x, y)-I_{2 \times 2}\right| \leq C_{0}\|\varphi\|_{C^{2}([a, b])},
$$

while by (3.23), (3.27), and Theorem 3.8, for $x \in(a, b)$,

$$
\begin{aligned}
\left|\Psi_{s}(x, y)-(x, y)\right| & \leq 2|g(s, x)-x|+2|h(s, x)-w(x)| \\
& =2\left|\int_{a}^{x}\left(\partial_{x} g(s, r)-1\right) d r\right|+2\left|\int_{a}^{x}\left(\partial_{x} h(s, r)-w^{\prime}(r)\right) d r\right| \\
& \leq C_{0}\|\varphi\|_{C^{2}([a, b])},
\end{aligned}
$$

while for $x \notin(a, b), \Psi_{s}(x, y)=(x, y)$ by (3.23) and (3.27), since $\varphi=0$ outside $(a, b)$. Hence, for all $(x, y) \in \mathbb{R}^{2}$, we deduce that

$$
\left|D \Phi_{s}(x, y)-I_{2 \times 2}\right| \leq C_{0}\left(1+\frac{1}{\delta_{0}}\right)\|\varphi\|_{C^{2}([a, b])}<1,
$$

provided $\|\varphi\|_{C^{2}([a, b])}<\delta_{0}^{2}$ and

$$
0<\delta_{0}<\frac{1}{2 C_{0}}
$$

This implies that $\Phi_{s}$ is invertible in $\mathbb{R}^{2}$. It follows by the inverse function theorem that $\Phi_{s}\left(\mathbb{R}^{2}\right)$ is open and $\left(\Phi_{s}\right)^{-1}$ is of class $C^{2}$.

Moreover, we have already seen that $\Psi_{s}(x, y)=(x, y)$ for $x \notin(a, b)$, and so, again by (3.38), $\Phi_{s}(x, y)=(x, y)$ for $x \notin(a, b)$. This shows that $\Phi_{s}$ is the identity outside $U$. In particular, $\Phi_{s}(\partial U)=\partial U$ and $\Phi_{s}(U) \subset U$.

Finally, we observe that

$$
\Phi_{s}=\Psi_{s} \quad \text { on } \Gamma_{s}
$$

since, by (3.36) and (3.37),

$$
L+\delta_{0} \leq 2 L-\|\varphi\|_{C^{0}([a, b])} \leq w(x)+s \varphi(x) \leq M+\|\varphi\|_{C^{0}([a, b])} \leq M+2-\delta_{0},
$$

provided

$$
\|\varphi\|_{C^{0}([a, b])} \leq \min \left\{1, L-\delta_{0}\right\}
$$


To conclude the proof, it remains to show (3.4). By (3.38), (3.39), and the fact that $\lambda(y)=1$ if $L+\delta_{0} \leq y \leq M+2-\delta_{0}$, we have that

$$
\begin{aligned}
& \dot{\Phi}_{s}(x, w(x)) \cdot\left(D \Phi_{s}(x, w(x)) \tau(x, w(x))\right) \\
& =\dot{\Psi}_{s}(x, w(x)) \cdot\left(D \Psi_{s}(x, w(x)) \tau(x, w(x))\right) \\
& =\left(\partial_{s} g(s, x), \partial_{s} h(s, x)\right) \cdot\left(\begin{array}{cc}
\partial_{x} g(s, x) & 0 \\
\partial_{x} h(s, x)-w^{\prime}(x) & 1
\end{array}\right) \frac{\left(1, w^{\prime}(x)\right)}{\sqrt{1+\left(w^{\prime}(x)\right)^{2}}}
\end{aligned}
$$

provided $\|\varphi\|_{C^{2, \alpha}(a, b)}$ is sufficiently small. Hence, by (2.3), (3.4) is equivalent to

$$
\partial_{x} g(s, x) \partial_{s} g(s, x)+\partial_{x} h(s, x) \partial_{s} h(s, x)=0
$$

for every $(s, x) \in[0,1] \times[a, b]$.

Differentiating (3.26) with respect to $x$ and $s$, respectively, yields

$$
\partial_{x} h=\left(w^{\prime}(g)+s \varphi^{\prime}(g)\right) \partial_{x} g, \quad \partial_{s} h=\left(w^{\prime}(g)+s \varphi^{\prime}(g)\right) \partial_{s} g+\varphi(g),
$$

so that

$$
\partial_{x} g \partial_{s} g+\partial_{x} h \partial_{s} h=\left[\left(1+\left(w^{\prime}(g)+s \varphi^{\prime}(g)\right)^{2}\right) \partial_{s} g+\left(w^{\prime}(g)+s \varphi^{\prime}(g)\right) \varphi(g)\right] \partial_{x} g,
$$

which is equal to 0 by (5.6), (5.11), and (5.16) in the Appendix.

\section{Proof of Theorem 1.1}

To prove Theorem 1.1 we first establish a minimality property with respect to special variations of the domain $\Omega_{+}$. To be precise, we will show the following result.

Theorem 4.1 Under the assumptions of Theorem 1.1, there exists $\delta_{1}>0$ such that for all polynomials $\varphi:[a, b] \rightarrow \mathbb{R}$ satisfying (3.2), extended to be zero outside $[a, b]$ and with $\|\varphi\|_{C^{2, \alpha}(a, b)} \leq \delta_{1}$,

$$
\mathcal{F}(u) \leq \mathcal{F}(v)
$$

for every $v \in \mathcal{A}$ such that $\{v>0\}=\Phi_{1}(\{u>0\})$, where $\left\{\Phi_{s}\right\}_{s \in[0,1]}$ is the admissible flow given in Theorem 3.1

We begin with some preliminary estimates.

Proposition 4.2 Let $Q \in C^{1,1}(\Omega)$, let $\varphi$ be as in Theorem 4.1. and let $u_{s}$ be the solution to problem (2.1), where $\Phi_{s}$ is given by (3.38). Then

$$
\left.\left|Q^{2}(x, w(x)+s \varphi(x))-\right| \nabla u_{s}(x, w(x)+s \varphi(x))\right|^{2} \mid \leq C\|\varphi\|_{C^{2, \alpha}(a, b)}
$$

and

$$
\left|\partial_{x}\left(Q^{2}(x, w(x)+s \varphi(x))-\left|\nabla u_{s}(x, w(x)+s \varphi(x))\right|^{2}\right)\right| \leq C\|\varphi\|_{C^{2, \alpha}(a, b)}
$$

for all $x \in[-1,1]$, where $C$ depends only on $\|w\|_{C^{2, \alpha}(-1,1)}$ and $\|u\|_{C^{2, \alpha}\left(\Omega_{+}\right)}$. 
Proof. The proof is subdivided into three steps.

Step 1: Recall that the function $\hat{u}_{s}:=u_{s} \circ \Phi_{s}$ satisfies the boundary value problem (2.7) with coefficients $A_{s}$ given by (2.8). Using the matrix expansion

$$
\left(I_{2 \times 2}+B\right)^{-1}=I_{2 \times 2}-B+o(|B|),
$$

it follows from (3.40) that the matrix $B_{s}:=A_{s}-I_{2 \times 2}$ satisfies

$$
\left|B_{s}\right| \leq C\|\varphi\|_{C^{2}([a, b])} \leq \frac{1}{2},
$$

provided $\|\varphi\|_{C^{2}([a, b])}$ is sufficiently small. In turn, the matrix $A_{s}$ is positive definite uniformly with respect to $s$. Using (4.1), by (2.7) and Poincaré inequality in the Lipschitz domain $\Omega_{+}$we obtain

$$
\left\|\hat{u}_{s}\right\|_{H^{1}\left(\Omega_{+}\right)} \leq C\|u\|_{H^{1}\left(\Omega_{+}\right)},
$$

where $C>0$ depends on $\Omega_{+}$but not on $s$. On the other hand, by (1.9) and (2.7) we have

$$
\begin{cases}\operatorname{div}\left(A_{s} \nabla\left(\hat{u}_{s}-u\right)\right)=-\operatorname{div}\left(B_{s} \nabla u\right) & \text { in } \Omega_{+}, \\ \hat{u}_{s}-u=0 & \text { on } \Gamma \cup\left(\{y=0\} \cap \partial \Omega_{+}\right),\end{cases}
$$

with $\left(\hat{u}_{s}-u\right)(-1, y)=\left(\hat{u}_{s}-u\right)(1, y)$ for all $( \pm 1, y) \in \overline{\Omega_{+}}$. Hence, with similar estimates, it follows from (4.1) that

$$
\left\|\hat{u}_{s}-u\right\|_{H^{1}\left(\Omega_{+}\right)} \leq C\left\|B_{s}\right\|_{C^{0}\left(\Omega_{+}\right)}\|u\|_{H^{1}\left(\Omega_{+}\right)} \leq C\|\varphi\|_{C^{2}([a, b])}\|u\|_{H^{1}\left(\Omega_{+}\right)} .
$$

Using the fact that $\hat{u}_{s}(x, y)=u_{s}(x, y)$ for all $y<L$, where $L$ is given in (3.36), by (4.2) and (4.3) we have

$$
\left\|u_{s}\right\|_{H^{1}((-1,1) \times(0, L))} \leq C, \quad\left\|u_{s}-u\right\|_{H^{1}((-1,1) \times(0, L))} \leq C\|\varphi\|_{C^{2}([a, b])},
$$

where $C$ depends on $\|w\|_{C^{1}(-1,1)}$ and $\|u\|_{H^{1}\left(\Omega_{+}\right)}$. By [GT, Theorem 9.13] and (4.4),

$$
\left\|u_{s}\right\|_{H^{2}\left((-1,1) \times\left(\varepsilon_{0}, 6 \varepsilon_{0}\right)\right)} \leq C, \quad\left\|u_{s}-u\right\|_{H^{2}\left((-1,1) \times\left(\varepsilon_{0}, 6 \varepsilon_{0}\right)\right)} \leq C\|\varphi\|_{C^{2}([a, b])}
$$

for $0<\varepsilon_{0}<L / 6$. Since $u_{s}$ and $u$ are periodic in the $x$ variable, they are still harmonic in $\mathbb{R} \times\left(\varepsilon_{0}, 6 \varepsilon_{0}\right)$ and satisfy

$$
\left\|u_{s}\right\|_{H^{2}\left(\left(a^{\prime}, b^{\prime}\right) \times\left(\varepsilon_{0}, 6 \varepsilon_{0}\right)\right)} \leq C, \quad\left\|u_{s}-u\right\|_{H^{2}\left(\left(a^{\prime}, b^{\prime}\right) \times\left(\varepsilon_{0}, 6 \varepsilon_{0}\right)\right)} \leq C\|\varphi\|_{C^{2}([a, b])}
$$

for some $a^{\prime}<-1<1<b^{\prime}$. Using [GT, Theorem 2.10] in the set $\left(a^{\prime}, b^{\prime}\right) \times\left(\varepsilon_{0}, 6 \varepsilon_{0}\right)$ we obtain that

$$
\left\|u_{s}\right\|_{C^{3}\left((-1,1) \times\left(2 \varepsilon_{0}, 5 \varepsilon_{0}\right)\right)} \leq C, \quad\left\|u_{s}-u\right\|_{C^{3}\left((-1,1) \times\left(2 \varepsilon_{0}, 5 \varepsilon_{0}\right)\right)} \leq C\|\varphi\|_{C^{2}([a, b])},
$$

where we invoked the continuous immersion of $H^{2}\left(\left(a^{\prime}, b^{\prime}\right) \times\left(\varepsilon_{0}, 6 \varepsilon_{0}\right)\right)$ into $C^{0}\left(\left(a^{\prime}, b^{\prime}\right) \times\right.$ $\left.\left(\varepsilon_{0}, 6 \varepsilon_{0}\right)\right)$. 
Step 2: Let $\|\varphi\|_{C^{2}([a, b])}<\varepsilon_{0}$. By Theorem 3.1 the function

$$
v_{s}(x, y):=u_{s}(x, y+s \varphi(x))
$$

is well-defined in the set

$$
\Omega_{0}:=\Omega_{+} \cap\left((-1,1) \times\left(3 \varepsilon_{0}, \infty\right)\right),
$$

and by (2.1) it satisfies the elliptic equation

$$
\partial_{x}^{2} v_{s}+\left(1+\left(s \varphi^{\prime}\right)^{2}\right) \partial_{y}^{2} v_{s}-2 s \varphi^{\prime} \partial_{x y}^{2} v_{s}-s \varphi^{\prime \prime} \partial_{y} v_{s}=0 \quad \text { in } \Omega_{0} .
$$

Moreover, since $\varphi=0$ outside $[a, b] \subset(-1,1)$, we have $v_{s}(-1, y)=u_{s}(-1, y)=$ $u_{s}(1, y)=v_{s}(1, y)$. Hence, $v_{s}$ satisfies the previous equation in $\left(\left(a^{\prime}, b^{\prime}\right) \times\left(3 \varepsilon_{0}, \infty\right)\right) \cap$ $\{u>0\}$, where $u$ has been extended periodically and $a^{\prime}<-1<1<b^{\prime}$. Moreover, $v_{s}=0$ on $\Gamma$ by (2.1) and (3.3), while $v_{s}\left(x, 3 \varepsilon_{0}\right)=u_{s}\left(x, 3 \varepsilon_{0}+s \varphi(x)\right)$. Since $\|\varphi\|_{C^{2}([a, b])}<\varepsilon_{0}$, we have that $\left(x, 3 \varepsilon_{0}+s \varphi(x)\right) \in\left(a^{\prime}, b^{\prime}\right) \times\left(2 \varepsilon_{0}, 4 \varepsilon_{0}\right)$.

By (3.40) and (4.2) we have that

$$
\left\|u_{s}\right\|_{H^{1}\left(\Phi_{s}\left(\Omega_{+}\right)\right)} \leq C,
$$

where $C$ depends on $\Omega_{+}$and $\|u\|_{H^{1}\left(\Omega_{+}\right)}$. By the lateral periodicity of $u_{s}$, the same estimate holds with $\Phi_{s}\left(\Omega_{+}\right)$replaced by $\Phi_{s}\left(\left(\left(a^{\prime}, b^{\prime}\right) \times\left(3 \varepsilon_{0}, \infty\right)\right) \cap\{u>0\}\right)$. In turn, by (4.6) and the chain rule

$$
\left\|v_{s}\right\|_{H^{1}\left(\left(\left(a^{\prime}, b^{\prime}\right) \times\left(3 \varepsilon_{0}, \infty\right)\right) \cap\{u>0\}\right)} \leq C .
$$

It follows from [GT, Theorem 9.13], with $T$ the graph of $w$ restricted to $\left(a^{\prime}, b^{\prime}\right)$, that

$$
\left\|v_{s}\right\|_{H^{2}\left(\left(\left(a^{\prime \prime}, b^{\prime \prime}\right) \times\left(4 \varepsilon_{0}, \infty\right)\right) \cap\{u>0\}\right)} \leq C
$$

for $a^{\prime}<a^{\prime \prime}<-1<1<b^{\prime \prime}<b^{\prime}$. By the continuous immersion of $H^{2}\left(\left(\left(a^{\prime \prime}, b^{\prime \prime}\right) \times\right.\right.$ $\left.\left.\left(4 \varepsilon_{0}, \infty\right)\right) \cap\{u>0\}\right)$ into $C^{0, \alpha}\left(\left(\left(a^{\prime \prime}, b^{\prime \prime}\right) \times\left(4 \varepsilon_{0}, \infty\right)\right) \cap\{u>0\}\right)$, we have

$$
\left\|v_{s}\right\|_{C^{0, \alpha}\left(\left(\left(a^{\prime \prime}, b^{\prime \prime}\right) \times\left(4 \varepsilon_{0}, \infty\right)\right) \cap\{u>0\}\right)} \leq C .
$$

By [GT, Corollary 6.7], with $T$ the graph of $w$ restricted to $\left(a^{\prime \prime}, b^{\prime \prime}\right)$, and using a covering argument, we obtain that there exists an $\varepsilon_{1}$-neighborhood $\Gamma_{1}$ of $\Gamma$ such that

$$
\left\|v_{s}\right\|_{C^{2, \alpha}\left(\Gamma_{1} \cap \Omega_{0}\right)} \leq C
$$

for some $0<\varepsilon_{1}<\varepsilon_{0}$. By (4.5) and the chain rule, we have that

$$
\left\|v_{s}\right\|_{C^{2, \alpha}\left((-1,1) \times\left(3 \varepsilon_{0}, 4 \varepsilon_{0}\right)\right)} \leq C .
$$

In the remaining set we can now use the interior Schauder's estimate in GT, Corollary 6.3] to conclude, also by (4.9) and (4.10), that there exists a constant $C$ depending only on $\|w\|_{C^{2, \alpha}(-1,1)}$ and $\|u\|_{H^{1}\left(\Omega_{+}\right)}$such that

$$
\left\|v_{s}\right\|_{C^{2, \alpha}\left(\Omega_{0}\right)} \leq C
$$


for all $s \in(0,1)$.

By (1.9) and (4.8),

$$
\Delta\left(v_{s}-u\right)=-\left(s \varphi^{\prime}\right)^{2} \partial_{y}^{2} v_{s}+2 s \varphi^{\prime} \partial_{x y}^{2} v_{s}+s \varphi^{\prime \prime} \partial_{y} v_{s} \quad \text { in } \Omega_{0} .
$$

Since $v_{s}-u=0$ on $\Gamma$, we can argue as in Step 1, and from standard estimates, Poincaré inequality, [4.11), and the fact that $\|\varphi\|_{C^{2}([a, b])}<\varepsilon_{0}$, we obtain

$$
\begin{aligned}
\left\|v_{s}-u\right\|_{H^{1}\left(\Omega_{0}\right)} & \leq C\|\varphi\|_{C^{2}([a, b])}+C\left\|v_{s}-u\right\|_{C^{1}\left((-1,1) \times\left\{3 \varepsilon_{0}\right\}\right)} \\
& \leq C\|\varphi\|_{C^{2}([a, b])}
\end{aligned}
$$

where the last inequality follows from the chain rule and (4.5). By the lateral periodicity of $v_{s}$ and $u$, the same estimate holds with $\Omega_{0}$ replaced by $\left(\left(a^{\prime}, b^{\prime}\right) \times\left(3 \varepsilon_{0}, \infty\right)\right) \cap\{u>$ $0\}$. Again by [GT, Theorem 9.13], with $T$ the graph of $w$ restricted to $\left(a^{\prime}, b^{\prime}\right)$, we deduce that

$$
\begin{aligned}
\left\|v_{s}-u\right\|_{H^{2}\left(\left(\left(a^{\prime \prime}, b^{\prime \prime}\right) \times\left(4 \varepsilon_{0}, \infty\right)\right) \cap\{u>0\}\right) \leq} & C\left\|v_{s}-u\right\|_{H^{1}\left(\left(\left(a^{\prime}, b^{\prime}\right) \times\left(3 \varepsilon_{0}, \infty\right)\right) \cap\{u>0\}\right)} \\
& +C\|\varphi\|_{C^{2}([a, b])}\left\|v_{s}\right\|_{H^{2}\left(\left(\left(a^{\prime}, b^{\prime}\right) \times\left(3 \varepsilon_{0}, \infty\right)\right) \cap\{u>0\}\right)} \\
\leq & C\|\varphi\|_{C^{2}([a, b])}
\end{aligned}
$$

for $a^{\prime}<a^{\prime \prime}<-1<1<b^{\prime \prime}<b^{\prime}$, and where we have used the previous inequality and (4.11), which holds in $\left(\left(a^{\prime \prime}, b^{\prime \prime}\right) \times\left(3 \varepsilon_{0}, \infty\right)\right) \cap\{u>0\}$ by lateral periodicity.

By [GT, Corollary 6.7], with $T$ the graph of $w$ restricted to $\left(a^{\prime \prime}, b^{\prime \prime}\right)$, and a covering argument, we have that

$$
\begin{aligned}
\left\|v_{s}-u\right\|_{C^{2, \alpha}\left(\Gamma_{1} \cap \Omega_{0}\right)} \leq & C\left\|v_{s}-u\right\|_{C^{0}\left(\left(\left(a^{\prime \prime}, b^{\prime \prime}\right) \times\left(3 \varepsilon_{0}, \infty\right)\right) \cap\{u>0\}\right)} \\
& +C\|\varphi\|_{C^{2, \alpha}([a, b])}\left\|v_{s}\right\|_{C^{2, \alpha}\left(\left(\left(a^{\prime \prime}, b^{\prime \prime}\right) \times\left(3 \varepsilon_{0}, \infty\right)\right) \cap\{u>0\}\right)} \\
\leq & C\|\varphi\|_{C^{2}([a, b])},
\end{aligned}
$$

where we used the fact that the estimate (4.11) holds in $\left(\left(a^{\prime \prime}, b^{\prime \prime}\right) \times\left(3 \varepsilon_{0}, \infty\right)\right) \cap\{u>0\}$ by lateral periodicity. We can now continue as before using (4.5) and GT, Corollary 6.3] to conclude that

$$
\left\|v_{s}-u\right\|_{C^{2, \alpha}\left(\Omega_{0}\right)} \leq C\|\varphi\|_{C^{2, \alpha}(a, b)} .
$$

Step 3: Since

$$
Q^{2}(x, w(x))-|\nabla u(x, w(x))|^{2}=0
$$

for all $x \in[-1,1]$, by (1.9) and (1.11) it follows that

$$
\begin{aligned}
\left.\left|Q^{2}(x, w(x)+s \varphi(x))-\right| \nabla u_{s}(x, w(x)+s \varphi(x))\right|^{2} \mid \\
\leq \mid \\
\quad\left|Q^{2}(x, w(x)+s \varphi(x))-Q^{2}(x, w(x))\right| \\
\quad+\left.|| \nabla u_{s}(x, w(x)+s \varphi(x))\right|^{2}-|\nabla u(x, w(x))|^{2} \mid \\
\leq|| Q^{2}\left\|_{C^{1}}\right\| \varphi \|_{C^{0}}+\left.|| \nabla u_{s}(x, w(x)+s \varphi(x))\right|^{2}-|\nabla u(x, w(x))|^{2} \mid .
\end{aligned}
$$


By (4.6), (4.11), (4.12), and the chain rule, the last term on the right-hand side can be estimated from above by

$$
\begin{aligned}
& C\left(\left\|\nabla u_{s}\right\|_{C^{0}}+\|\nabla u\|_{C^{0}}\right)\left|\nabla u_{s}(x, w(x)+s \varphi(x))-\nabla u(x, w(x))\right| \\
& \leq C\left(\left\|\nabla u_{s}\right\|_{C^{0}}+\|\nabla u\|_{C^{0}}\right)\left(\left|\nabla v_{s}(x, w(x))-\nabla u(x, w(x))\right|+\left\|\nabla v_{s}\right\|_{C^{0}}\left|\varphi^{\prime}(x)\right|\right) \\
& \leq C\|\varphi\|_{C^{2, \alpha}(a, b)},
\end{aligned}
$$

where, as before, $C$ depends only on $\|w\|_{C^{2, \alpha}(-1,1)}$ and $\|u\|_{C^{1}\left(\Omega_{+}\right)}$. On the other hand by (4.13),

$$
\partial_{x}\left(Q^{2}(x, w(x))-|\nabla u(x, w(x))|^{2}\right)=0
$$

for all $x \in[-1,1]$, and so

$$
\begin{aligned}
\mid \partial_{x}\left(Q^{2}(\right. & \left.x, w(x)+s \varphi(x))-\left|\nabla u_{s}(x, w(x)+s \varphi(x))\right|^{2}\right) \mid \\
\leq & \left|\partial_{x}\left(Q^{2}(x, w(x)+s \varphi(x))-Q^{2}(x, w(x))\right)\right| \\
& \quad+\left|\partial_{x}\left(\left|\nabla u_{s}(x, w(x)+s \varphi(x))\right|^{2}-|\nabla u(x, w(x))|^{2}\right)\right| \\
\leq & C\left\|Q^{2}\right\|_{C^{1,1}}\|\varphi\|_{C^{1}}+\left|\partial_{x}\left(\left|\nabla u_{s}(x, w(x)+s \varphi(x))\right|^{2}-|\nabla u(x, w(x))|^{2}\right)\right|,
\end{aligned}
$$

where $C$ depends only on $\|w\|_{C^{1}(-1,1)}$. The last term on the right-hand side can be estimated from above by

$$
\begin{aligned}
& C\left\|\nabla u_{s}\right\|_{C^{0}}\left|\nabla^{2} u_{s}(x, w(x)+s \varphi(x))-\nabla^{2} u(x, w(x))\right| \\
& +C\left\|\nabla^{2} u\right\|_{C^{0}}\left|\nabla u_{s}(x, w(x)+s \varphi(x))-\nabla u(x, w(x))\right|+C\|u\|_{C^{2}}^{2}\left|\varphi^{\prime}(x)\right|,
\end{aligned}
$$

where, as before, $C$ depends only on $\|w\|_{C^{2, \alpha}(-1,1)}$ and $\|u\|_{C^{2}\left(\Omega_{+}\right)}$. By (4.6) and the chain rule, we have that

$$
\begin{aligned}
& \left|\nabla^{2} u_{s}(x, w(x)+s \varphi(x))-\nabla^{2} u(x, w(x))\right| \\
& \leq\left|\nabla^{2} v_{s}(x, w(x))-\nabla^{2} u(x, w(x))\right|+C\left\|v_{s}\right\|_{C^{2, \alpha}}\|\varphi\|_{C^{2}([a, b])} \leq C\|\varphi\|_{C^{2, \alpha}(a, b)},
\end{aligned}
$$

where in the last inequality we used (4.11) and (4.12). A similar estimate holds for $\left|\nabla u_{s}(x, w(x)+s \varphi(x))-\nabla u(x, w(x))\right|$. This concludes the proof.

Remark 4.3 The proof of the previous proposition could be significantly simplified if we could show that the diffeomorphism $\Phi_{s}$ is of class $C^{2, \alpha}$ rather than just $C^{2}$, and if we had uniform estimates on the $C^{2, \alpha}$ norm of $\Phi_{s}$ in terms of $\|w\|_{C^{2, \alpha}(-1,1)}$ and $\|\varphi\|_{C^{2, \alpha}(a, b)}$. Indeed, the $C^{2, \alpha}$ bounds on $u_{s}$ and $v_{s}$ would follow in this case from standard elliptic estimates.

Next we estimate the second integral on the right-hand side of (2.13).

Proposition 4.4 Let $Q \in C^{1,1}(\Omega)$, let $\varphi$ be as in Theorem 4.1, and let $u_{s}$ be the solution to problem (2.1), where $\Phi_{s}$ is given by (3.38). Then there exists $C>0$, depending only on $\|w\|_{C^{2, \alpha}(-1,1)}$ and $\|u\|_{C^{2}\left(\Omega_{+}\right)}$, such that for every $s \in[0,1]$ and every $\psi \in C\left(\Gamma_{s}\right)$,

$$
\left|\int_{\Gamma_{s}} \kappa_{s}\left(\partial_{\nu_{s}} u_{s}\right)^{2} \psi^{2} d \mathcal{H}^{1}-\int_{\Gamma} \kappa\left(\partial_{\nu} u\right)^{2} \psi^{2} \circ \Phi_{s} d \mathcal{H}^{1}\right| \leq C\|\varphi\|_{C^{2}([a, b])} \int_{\Gamma_{s}} \psi^{2} d \mathcal{H}^{1}
$$


and

$$
\left|\int_{\Gamma_{s}} \partial_{\nu_{s}} Q^{2} \psi^{2} d \mathcal{H}^{1}-\int_{\Gamma} \partial_{\nu} Q^{2} \psi^{2} \circ \Phi_{s} d \mathcal{H}^{1}\right| \leq C\|\varphi\|_{C^{1}([a, b])} \int_{\Gamma_{s}} \psi^{2} d \mathcal{H}^{1} .
$$

Proof. Let $v_{s}$ and $\Omega_{0}$ be defined as in (4.6) and (4.7). Then, by (4.11) and (4.12),

$$
\left\|v_{s}\right\|_{C^{2, \alpha}\left(\Omega_{0}\right)} \leq C, \quad\left\|v_{s}-u\right\|_{C^{2, \alpha}\left(\Omega_{0}\right)} \leq C\|\varphi\|_{C^{2, \alpha}(a, b)}
$$

for some constant $C>0$ depending only on $\|w\|_{C^{2, \alpha}(-1,1)}$ and $\|u\|_{C^{2}\left(\Omega_{+}\right)}$. By the chain rule,

$$
\begin{aligned}
\partial_{\nu_{s}} u_{s}(x, w+s \varphi)=\nabla u_{s}(x, w+s \varphi) \cdot \nu_{s}(x, w+s \varphi) \\
=\nabla v_{s}(x, w) \cdot \nu(x, w)-s \varphi^{\prime} \partial_{y} v_{s}(x, w) e_{1} \cdot \nu_{s}(x, w+s \varphi) \\
\quad+\nabla v_{s}(x, w) \cdot\left(\nu_{s}(x, w+s \varphi)-\nu(x, w)\right) .
\end{aligned}
$$

Using (4.16),

$$
\left|\partial_{\nu_{s}} u_{s}(x, w+s \varphi)-\partial_{\nu} u(x, w)\right| \leq\left|\partial_{\nu} v_{s}(x, w)-\partial_{\nu} u(x, w)\right|+C\|\varphi\|_{C^{1}([a, b])},
$$

where to estimate $\left|\nu_{s}(x, w+s \varphi)-\nu(x, w)\right|$ we used the fact that the function $t \mapsto \frac{1}{\sqrt{1+t^{2}}}$ is 1-Lipschitz. Similarly,

$$
\begin{aligned}
\left|\kappa_{s}(x, w+s \varphi)-\kappa(x, w)\right| & =\left|\frac{w^{\prime \prime}+s \varphi^{\prime \prime}}{\left(1+\left(w^{\prime}+s \varphi^{\prime}\right)^{2}\right)^{3 / 2}}-\frac{w^{\prime \prime}}{\left(1+\left(w^{\prime}\right)^{2}\right)^{3 / 2}}\right| \\
& \leq C\|\varphi\|_{C^{2}([a, b])} .
\end{aligned}
$$

Combining (4.16), (4.17), and (4.18), and using a change of variable, we obtain (4.14).

On the other hand,

$$
\begin{aligned}
\partial_{\nu_{s}} Q^{2}(x, w+s \varphi)-\partial_{\nu} Q^{2}(x, w)= & \nabla Q^{2}(x, w+s \varphi) \cdot\left(\nu_{s}(x, w+s \varphi)-\nu(x, w)\right) \\
& +\left(\nabla Q^{2}(x, w+s \varphi)-\nabla Q^{2}(x, w)\right) \cdot \nu(x, w),
\end{aligned}
$$

and so

$$
\left|\partial_{\nu_{s}} Q^{2}(x, w+s \varphi)-\partial_{\nu} Q^{2}(x, w)\right| \leq C\|\varphi\|_{C^{1}([a, b])},
$$

which gives 44.15).

We now estimate the first integral on the right-hand side of (2.13).

Proposition 4.5 Let $\varphi$ be as in Theorem 4.1, and let $u_{s}$ be the solution to problem (2.1), where $\Phi_{s}$ is given by (3.38). Then there exists $C>0$, depending only on $\|w\|_{C^{2, \alpha}(-1,1)}$ and $\|u\|_{C^{2}\left(\Omega_{+}\right)}$, such that for every $s \in[0,1]$ and every $\psi \in C^{1}\left(\Gamma_{s}\right)$,

$$
\left.\left|\int_{\Phi_{s}\left(\Omega_{+}\right)}\right| \nabla u_{\psi}^{s}\right|^{2} d \boldsymbol{x}-\int_{\Omega_{+}}\left|\nabla u_{\psi \circ \Phi_{s}}^{0}\right|^{2} d \boldsymbol{x} \mid \leq C\|\varphi\|_{C^{2, \alpha}(a, b)}\|\psi\|_{H^{1 / 2}\left(\Gamma_{s}\right)}^{2},
$$


where $u_{\psi}^{s}$ is the unique solution to the problem

$$
\begin{cases}\Delta u_{\psi}^{s}=0 & \text { in } \Phi_{s}\left(\Omega_{+}\right) \\ u_{\psi}^{s}=-\psi \partial_{\nu_{s}} u_{s} & \text { on } \Gamma_{s} \\ u_{\psi}^{s}=0 & \text { on } \Phi_{s}\left(\{y=0\} \cap \partial \Omega_{+}\right) .\end{cases}
$$

with $u_{\psi}^{s}(-1, y)=u_{\psi}^{s}(1, y)$ for all $y$ such that $( \pm 1, y) \in \Phi_{s}\left(\overline{\Omega_{+}}\right)$.

Proof. Reasoning as in the proof of (2.20), we have

$$
\int_{\Phi_{s}\left(\Omega_{+}\right)}\left|\nabla u_{\psi}^{s}\right|^{2} d \boldsymbol{x}=\int_{\Gamma_{s}} u_{\psi}^{s} \partial_{\nu_{s}} u_{\psi}^{s} d \mathcal{H}^{1}
$$

Define

$$
\hat{u}_{\psi}^{s}:=u_{\psi}^{s} \circ \Phi_{s}
$$

Then $\hat{u}_{\psi}^{s}$ satisfies

$$
\begin{cases}\operatorname{div}\left(A_{s} \nabla \hat{u}_{\psi}^{s}\right)=0 & \text { in } \Omega_{+} \\ \hat{u}_{\psi}^{s}=-\left(\psi \partial_{\nu_{s}} u_{s}\right) \circ \Phi_{s} & \text { on } \Gamma \\ \hat{u}_{\psi}^{s}=0 & \text { on } \overline{\Omega_{+}} \cap\{y=0\}\end{cases}
$$

with $\hat{u}_{\psi}^{s}(-1, y)=\hat{u}_{\psi}^{s}(1, y)$ for all $y$ such that $( \pm 1, y) \in \overline{\Omega_{+}}$, where $A_{s}$ is given by (2.8). Multiplying the first equation in (4.19) by $\hat{u}_{\psi}^{s}$ and by the divergence theorem, we obtain

$$
\begin{aligned}
\int_{\Omega_{+}}\left(A_{s} \nabla \hat{u}_{\psi}^{s}\right) \cdot \nabla \hat{u}_{\psi}^{s} d \boldsymbol{x} & \leq\left\|\left(A_{s} \nabla \hat{u}_{\psi}^{s}\right) \cdot \nu\right\|_{H^{-1 / 2}(\Gamma)}\left\|\left(\psi \partial_{\nu_{s}} u_{s}\right) \circ \Phi_{s}\right\|_{H^{1 / 2}(\Gamma)} \\
& \leq C\left\|A_{s} \nabla \hat{u}_{\psi}^{s}\right\|_{L^{2}\left(\Omega_{+}\right)}\left\|\left(\psi \partial_{\nu_{s}} u_{s}\right) \circ \Phi_{s}\right\|_{H^{1 / 2}(\Gamma)} \\
& \leq C\left\|\nabla \hat{u}_{\psi}^{s}\right\|_{L^{2}\left(\Omega_{+}\right)}\left\|\left(\psi \partial_{\nu_{s}} u_{s}\right) \circ \Phi_{s}\right\|_{H^{1 / 2}(\Gamma)},
\end{aligned}
$$

where we used (4.1) and the continuity of the normal trace in the space $H\left(\operatorname{div} ; \Omega_{+}\right)$ (see, e.g., $\left[\mathrm{BF}\right.$, Section 3.2]), and where the constant $C$ depends only on $\Omega_{+}$. The previous estimate, together with (3.40) and (4.1), implies that

$$
\begin{aligned}
\left\|\nabla \hat{u}_{\psi}^{s}\right\|_{L^{2}\left(\Omega_{+}\right)} & \leq C\left\|\left(\psi \partial_{\nu_{s}} u_{s}\right) \circ \Phi_{s}\right\|_{H^{1 / 2}(\Gamma)} \leq C\left\|\psi \partial_{\nu_{s}} u_{s}\right\|_{H^{1 / 2}\left(\Gamma_{s}\right)} \\
& \leq C\|\psi\|_{L^{2}\left(\Gamma_{s}\right)}\left\|\partial_{\nu_{s}} u_{s}\right\|_{C^{0,1}\left(\Gamma_{s}\right)}+C|\psi|_{H^{1 / 2}\left(\Gamma_{s}\right)}\left\|\partial_{\nu_{s}} u_{s}\right\|_{C^{0}\left(\Gamma_{s}\right)} \\
& \leq C\|\psi\|_{H^{1 / 2}\left(\Gamma_{s}\right)},
\end{aligned}
$$

where in the last inequality we reasoned as in the proof of Proposition 4.4 and used (4.16). By the Poincaré inequality, we get

$$
\left\|\hat{u}_{\psi}^{s}\right\|_{H^{1}\left(\Omega_{+}\right)} \leq C\|\psi\|_{H^{1 / 2}\left(\Gamma_{s}\right)} .
$$

On the other hand,

$$
\begin{cases}\operatorname{div}\left(A_{s} \nabla\left(\hat{u}_{\psi}^{s}-u_{\psi \circ \Phi_{s}}^{0}\right)\right)=-\operatorname{div}\left(B_{s} \nabla u_{\psi \circ \Phi_{s}}^{0}\right) & \text { in } \Omega_{+}, \\ \hat{u}_{\psi}^{s}-u_{\psi \circ \Phi_{s}}^{0}=-\left(\psi \partial_{\nu_{s}} u_{s}\right) \circ \Phi_{s}+\left(\psi \circ \Phi_{s}\right) \partial_{\nu} u & \text { on } \Gamma \\ \hat{u}_{\psi}^{s}-u_{\psi \circ \Phi_{s}}^{0}=0 & \text { on }\{y=0\} \cap \partial \Omega_{+},\end{cases}
$$


with $\left(\hat{u}_{\psi}^{s}-u_{\psi_{\circ} \Phi_{s}}^{0}\right)(-1, y)=\left(\hat{u}_{\psi}^{s}-u_{\psi \circ \Phi_{s}}^{0}\right)(1, y)$ for all $y$ such that $( \pm 1, y) \in \overline{\Omega_{+}}$, where $B_{s}=A_{s}-I_{2 \times 2}$. Reasoning as before, by (4.1) and (4.20) we get

$$
\begin{aligned}
& \left\|\nabla\left(\hat{u}_{\psi}^{s}-u_{\psi \circ \Phi_{s}}^{0}\right)\right\|_{L^{2}\left(\Omega_{+}\right)}^{2} \leq\left\|B_{s} \nabla u_{\psi \circ \Phi_{s}}^{0}\right\|_{L^{2}\left(\Omega_{+}\right)}\left\|\nabla\left(\hat{u}_{\psi}^{s}-u_{\psi \circ \Phi_{s}}^{0}\right)\right\|_{L^{2}\left(\Omega_{+}\right)} \\
& \quad+\left\|\left(A_{s} \nabla\left(\hat{u}_{\psi}^{s}-u_{\psi \circ \Phi_{s}}^{0}\right)+B_{s} \nabla u_{\psi \circ \Phi_{s}}^{0}\right) \cdot \nu\right\|_{H^{-1 / 2}(\Gamma)}\left\|\left(\psi \circ \Phi_{s}\right)\left(\partial_{\nu} u-\partial_{\nu_{s}} u_{s} \circ \Phi_{s}\right)\right\|_{H^{1 / 2}(\Gamma)} \\
& \leq C\|\varphi\|_{C^{2}([a, b])}\|\psi\|_{H^{1 / 2}\left(\Gamma_{s}\right)}\left\|\nabla\left(\hat{u}_{\psi}^{s}-u_{\psi \circ \Phi_{s}}^{0}\right)\right\|_{L^{2}\left(\Omega_{+}\right)} \\
& \quad+C\left\|A_{s} \nabla\left(\hat{u}_{\psi}^{s}-u_{\psi \circ \Phi_{s}}^{0}\right)+B_{s} \nabla u_{\psi \circ \Phi_{s}}^{0}\right\|_{L^{2}\left(\Omega_{+}\right)}\left\|\left(\psi \circ \Phi_{s}\right)\left(\partial_{\nu} u-\partial_{\nu_{s}} u_{s} \circ \Phi_{s}\right)\right\|_{H^{1 / 2}(\Gamma)} \\
& \leq C\|\varphi\|_{C^{2}([a, b])}\|\psi\|_{H^{1 / 2}\left(\Gamma_{s}\right)}\left\|\nabla\left(\hat{u}_{\psi}^{s}-u_{\psi \circ \Phi_{s}}^{0}\right)\right\|_{L^{2}\left(\Omega_{+}\right)} \\
& \quad+C\left\|\nabla\left(\hat{u}_{\psi}^{s}-u_{\psi \circ \Phi_{s}}^{0}\right)\right\|_{L^{2}\left(\Omega_{+}\right)}\left\|\left(\psi \circ \Phi_{s}\right)\left(\partial_{\nu} u-\partial_{\nu_{s}} u_{s} \circ \Phi_{s}\right)\right\|_{H^{1 / 2}(\Gamma)} \\
& \quad+C\|\varphi\|_{C^{2}([a, b])}\|\psi\|_{H^{1 / 2}\left(\Gamma_{s}\right)}\left\|\left(\psi \circ \Phi_{s}\right)\left(\partial_{\nu} u-\partial_{\nu_{s}} u_{s} \circ \Phi_{s}\right)\right\|_{H^{1 / 2}(\Gamma)} .
\end{aligned}
$$

Hence,

$$
\begin{aligned}
\left\|\nabla\left(\hat{u}_{\psi}^{s}-u_{\psi \circ \Phi_{s}}^{0}\right)\right\|_{L^{2}\left(\Omega_{+}\right)} \leq & C\|\varphi\|_{C^{2}([a, b])}\|\psi\|_{H^{1 / 2}\left(\Gamma_{s}\right)} \\
& +C\left\|\left(\psi \circ \Phi_{s}\right)\left(\partial_{\nu} u-\partial_{\nu_{s}} u_{s} \circ \Phi_{s}\right)\right\|_{H^{1 / 2}(\Gamma)} .
\end{aligned}
$$

As before, using (4.16), we obtain

$$
\begin{aligned}
& \left\|\left(\psi \circ \Phi_{s}\right)\left(\partial_{\nu} u-\partial_{\nu_{s}} u_{s} \circ \Phi_{s}\right)\right\|_{H^{1 / 2}(\Gamma)} \leq C\|\psi\|_{L^{2}\left(\Gamma_{s}\right)}\left\|\partial_{\nu} u-\partial_{\nu_{s}} u_{s} \circ \Phi_{s}\right\|_{C^{0,1}(\Gamma)} \\
& \quad+C|\psi|_{H^{1 / 2}\left(\Gamma_{s}\right)}\left\|\partial_{\nu} u-\partial_{\nu_{s}} u_{s} \circ \Phi_{s}\right\|_{C^{0}(\Gamma)} \leq C\|\varphi\|_{C^{2, \alpha}(a, b)}\|\psi\|_{H^{1 / 2}\left(\Gamma_{s}\right)},
\end{aligned}
$$

and so

$$
\left\|\nabla \hat{u}_{\psi}^{s}-\nabla u_{\psi \circ \Phi_{s}}^{0}\right\|_{L^{2}\left(\Omega_{+}\right)} \leq C\|\varphi\|_{C^{2, \alpha}(a, b)}\|\psi\|_{H^{1 / 2}\left(\Gamma_{s}\right)} .
$$

Then, also by (4.20),

$$
\left.\left|\int_{\Omega_{+}}\right| \nabla \hat{u}_{\psi}^{s}\right|^{2} d \boldsymbol{x}-\int_{\Omega_{+}}\left|\nabla u_{\psi \circ \Phi_{s}}^{0}\right|^{2} d \boldsymbol{x} \mid \leq C\|\varphi\|_{C^{2, \alpha}(a, b)}\|\psi\|_{H^{1 / 2}\left(\Gamma_{s}\right)}^{2},
$$

By a change of variables, we get

$$
\int_{\Omega_{+}}\left|\nabla \hat{u}_{\psi}^{s}\right|^{2} d \boldsymbol{x}=\int_{\Phi_{s}\left(\Omega_{+}\right)}\left|\left(D \Phi_{s} \circ \Phi_{s}^{-1}\right) \nabla u_{\psi}^{s}\right|^{2} \operatorname{det} D \Phi_{s} d \boldsymbol{y}
$$

In turn, by (3.40) and (4.20), we deduce that

$$
\begin{aligned}
\left.\left|\int_{\Phi_{s}\left(\Omega_{+}\right)}\right| \nabla u_{\psi}^{s}\right|^{2} d \boldsymbol{x}-\int_{\Omega_{+}}\left|\nabla \hat{u}_{\psi}^{s}\right|^{2} d \boldsymbol{x} \mid & \leq C\|\varphi\|_{C^{2}([a, b])} \int_{\Phi_{s}\left(\Omega_{+}\right)}\left|\nabla u_{\psi}^{s}\right|^{2} d \boldsymbol{x} \\
& \leq C\|\varphi\|_{C^{2}([a, b])}\|\psi\|_{H^{1 / 2}\left(\Gamma_{s}\right)}^{2},
\end{aligned}
$$

and this concludes the proof.

Finally, we estimate the first term in the last integral on the right-hand side of (2.13). 
Proposition 4.6 Let $Q \in C^{1,1}(\Omega)$, let $\varphi$ be as in Theorem 4.1. and let $u_{s}$ be the solution to problem (2.1), where $\Phi_{s}$ is given by (3.38). If $\|\varphi\|_{C^{2, \alpha}(a, b)}$ is sufficiently small, then for every $s \in[0,1]$ the following inequality holds:

$$
\left|\int_{\Gamma_{s}}\left(Q^{2}-\left|\nabla u_{s}\right|^{2}\right) Z_{s} \cdot \nu_{s} d \mathcal{H}^{1}\right| \leq C\|\varphi\|_{C^{2, \alpha}(a, b)} \int_{\Gamma_{s}}\left(X_{s} \cdot \nu_{s}\right)^{2} d \mathcal{H}^{1},
$$

where $X_{s}$ and $Z_{s}$ are given in (2.3) and $C>0$ depends on $\|w\|_{C^{2, \alpha}(-1,1)}$.

Proof. Observe that, by (3.38), (3.42), and the fact that $\lambda(y)=1$ if $L+\delta_{0} \leq y \leq$ $M+2-\delta_{0}$, we have that

$$
\begin{aligned}
& \dot{\Phi}_{s}(x, w(x)) \cdot\left(D \Phi_{s}(x, w(x))\right)^{-T} \nu(x, w(x)) \\
& =\dot{\Psi}_{s}(x, w(x)) \cdot\left(D \Psi_{s}(x, w(x))\right)^{-T} \nu(x, w(x)) \\
& =-\frac{\partial_{x} h(s, x)}{\partial_{x} g(s, x)} \frac{\partial_{s} g(s, x)}{\sqrt{1+\left(w^{\prime}(x)\right)^{2}}}+\frac{\partial_{s} h(s, x)}{\sqrt{1+\left(w^{\prime}(x)\right)^{2}}}=\frac{\varphi(g(s, x))}{\sqrt{1+\left(w^{\prime}(x)\right)^{2}}}
\end{aligned}
$$

provided $\|\varphi\|_{C^{2, \alpha}(a, b)}$ is sufficiently small. Similarly,

$$
\begin{aligned}
& \ddot{\Phi}_{s}(x, w(x)) \cdot\left(D \Phi_{s}(x, w(x))\right)^{-T} \nu(x, w(x)) \\
& =\ddot{\Psi}_{s}(x, w(x)) \cdot\left(D \Psi_{s}(x, w(x))\right)^{-T} \nu(x, w(x)) \\
& =\frac{1}{\partial_{x} g(s, x)}\left(\partial_{s}^{2} g(s, x), \partial_{s}^{2} h(s, x)\right) \cdot\left(\begin{array}{cc}
1 & -\partial_{x} h(s, x)+w^{\prime}(x) \\
0 & \partial_{x} g(s, x)
\end{array}\right) \frac{\left(-w^{\prime}(x), 1\right)}{\sqrt{1+\left(w^{\prime}(x)\right)^{2}}} \\
& =-\frac{\partial_{x} h(s, x)}{\partial_{x} g(s, x)} \frac{\partial_{s}^{2} g(s, x)}{\sqrt{1+\left(w^{\prime}(x)\right)^{2}}}+\frac{\partial_{s}^{2} h(s, x)}{\sqrt{1+\left(w^{\prime}(x)\right)^{2}}}
\end{aligned}
$$

Differentiating $(3.42)_{2}$ with respect to $s$ gives

$$
\partial_{s}^{2} h=\left(w^{\prime \prime}(g)+s \varphi^{\prime \prime}(g)\right)\left(\partial_{s} g\right)^{2}+2 \varphi^{\prime}(g) \partial_{s} g+\left(w^{\prime}(g)+s \varphi^{\prime}(g)\right) \partial_{s}^{2} g .
$$

so that, by (3.42) 1 and (4.24),

$$
\begin{aligned}
& \ddot{\Phi}_{s}(x, w(x)) \cdot\left(D \Phi_{s}(x, w(x))\right)^{-T} \nu(x, w(x)) \\
& =\frac{\left(w^{\prime \prime}(g)+s \varphi^{\prime \prime}(g)\right)\left(\partial_{s} g\right)^{2}}{\sqrt{1+\left(w^{\prime}(x)\right)^{2}}}+\frac{2 \varphi^{\prime}(g) \partial_{s} g}{\sqrt{1+\left(w^{\prime}(x)\right)^{2}}} .
\end{aligned}
$$

Since, by (2.5), we have

$$
\nu_{s}\left(\Phi_{s}(x, w(x))\right)=\frac{\left(D \Phi_{s}(x, w(x))\right)^{-T} \nu(x, w(x))}{\left(\left(D \Phi_{s}(x, w(x))\right)^{-T} \nu(x, w(x))\right)}
$$

and

$$
\left(\left(D \Phi_{s}(x, w(x))\right)^{-T} \nu(x, w(x))\right)=\frac{\sqrt{1+\left[w^{\prime}(g(x))+s \varphi^{\prime}(g(x))\right]^{2}}}{\sqrt{1+\left(w^{\prime}(x)\right)^{2}}}
$$


by (2.3) we have

$$
\begin{aligned}
& \int_{\Gamma_{s}}\left(Q^{2}-\left|\nabla u_{s}\right|^{2}\right) Z_{s} \cdot \nu_{s} d \mathcal{H}^{1} \\
& =\int_{a}^{b}\left(Q^{2}(g, h)-\left|\nabla u_{s}(g, h)\right|^{2}\right)\left(w^{\prime \prime}(g)+s \varphi^{\prime \prime}(g)\right)\left(\partial_{s} g\right)^{2} \partial_{x} g d x \\
& \quad+\int_{a}^{b}\left(Q^{2}(g, h)-\left|\nabla u_{s}(g, h)\right|^{2}\right) 2 \varphi^{\prime}(g) \partial_{s} g \partial_{x} g d x=: I+I I .
\end{aligned}
$$

By (5.6) and (5.16) in the appendix we obtain

$$
\left|\partial_{s} g(s, x)\right| \leq|\varphi(g(s, x))|
$$

for every $(s, x) \in[0,1] \times[a, b]$. Hence, by Proposition 4.2 for $\|\varphi\|_{C^{2, \alpha}(a, b)}$ sufficiently small,

$$
\begin{aligned}
|I| & \leq C\|\varphi\|_{C^{2, \alpha}(a, b)} \int_{a}^{b} \varphi^{2}(g(s, x)) \partial_{x} g d x \\
& \leq C\|\varphi\|_{C^{2, \alpha}(a, b)} \int_{a}^{b} \frac{\varphi^{2}(g(s, x))}{\sqrt{1+\left[w^{\prime}(g)+s \varphi^{\prime}(g)\right]^{2}}} \partial_{x} g d x \\
& =C\|\varphi\|_{C^{2, \alpha}(a, b)} \int_{\Gamma_{s}}\left(X_{s} \cdot \nu_{s}\right)^{2} d \mathcal{H}^{1},
\end{aligned}
$$

where $C$ depends only on $\|w\|_{C^{2, \alpha}(a, b)}$, and where we have used (4.23).

To estimate $I I$, we use (5.6) to write

$$
I I=-\int_{a}^{b}\left(Q^{2}(g, h)-\left|\nabla u_{s}(g, h)\right|^{2}\right) \frac{2 \varphi^{\prime}(g) \varphi(g)\left(w^{\prime}(g)+s \varphi^{\prime}(g)\right) \partial_{x} g}{1+\left[w^{\prime}(g)+s \varphi^{\prime}(g)\right]^{2}} d x
$$

Using the change of variables $r=g(x, s)$ and (3.26), we have

$$
I I=-\int_{a}^{b} \frac{\left(Q^{2}(r, w+s \varphi)-\left|\nabla u_{s}(r, w+s \varphi)\right|^{2}\right) 2 \varphi^{\prime} \varphi\left(w^{\prime}+s \varphi^{\prime}\right)}{1+\left[w^{\prime}+s \varphi^{\prime}\right]^{2}} d r .
$$

Integrating by parts and using (3.2), we obtain

$$
I I=\int_{a}^{b} \varphi^{2} \partial_{r}\left(\frac{\left(Q^{2}(r, w+s \varphi)-\mid \nabla u_{s}\left(\left.(r, w+s \varphi)\right|^{2}\right)\left(w^{\prime}+s \varphi^{\prime}\right)\right.}{1+\left[w^{\prime}+s \varphi^{\prime}\right]^{2}}\right) d r .
$$

It follows from Proposition 4.2 that

$$
|I I| \leq C\|\varphi\|_{C^{2, \alpha}(a, b)} \int_{a}^{b} \varphi^{2} d r \leq C\|\varphi\|_{C^{2, \alpha}(a, b)} \int_{\Gamma_{s}}\left(X_{s} \cdot \nu_{s}\right)^{2} d \mathcal{H}^{1},
$$

where in the last inequality we have reasoned as in the estimate of $I$.

Next we prove Theorem 4.1 . 
Proof of Theorem 4.1. Let $\varphi$ be as in the statement of Theorem 4.1 and let $\left\{\Phi_{s}\right\}_{s \in[0,1]}$ be the admissible flow given in Theorem 3.1. By Theorem 2.3 and Propositions 4.2 and 4.6 .

$$
\begin{gathered}
\frac{d^{2}}{d s^{2}} \mathcal{F}\left(u_{s}\right) \geq \int_{\Phi_{s}\left(\Omega_{+}\right)} 2\left|\nabla \dot{u}_{s}\right|^{2} d \boldsymbol{x}+\int_{\Gamma_{s}}\left(\partial_{\nu_{s}} Q^{2}+2 \kappa_{s}\left(\partial_{\nu_{s}} u_{s}\right)^{2}\right)\left(X_{s} \cdot \nu_{s}\right)^{2} d \mathcal{H}^{1} \\
-C\|\varphi\|_{C^{2, \alpha}(a, b)} \int_{\Gamma_{s}}\left(X_{s} \cdot \nu_{s}\right)^{2} d \mathcal{H}^{1},
\end{gathered}
$$

where we used the fact that $\left|\kappa_{s}\right| \leq C$. On the other hand, by (2.6) and by Propositions 4.4 and 4.5 with $\psi=X_{s} \cdot \nu_{s}$, we have

$$
\begin{gathered}
\frac{d^{2}}{d s^{2}} \mathcal{F}\left(u_{s}\right) \geq \int_{\Omega_{+}} 2\left|\nabla u_{\psi_{s}}\right|^{2} d \boldsymbol{x}+\int_{\Gamma}\left(\partial_{\nu} Q^{2}+2 \kappa\left(\partial_{\nu} u\right)^{2}\right)\left(X_{s} \cdot \nu_{s}\right)^{2} \circ \Phi_{s} d \mathcal{H}^{1} \\
-C_{1}\|\varphi\|_{C^{2, \alpha}(a, b)}\left\|X_{s} \cdot \nu_{s}\right\|_{H^{1 / 2}\left(\Gamma_{s}\right)}^{2},
\end{gathered}
$$

where $\psi_{s}:=\left(X_{s} \cdot \nu_{s}\right) \circ \Phi_{s}$ and $u_{\psi_{s}}$ is the unique solution to the problem

$$
\begin{cases}\Delta u_{\psi_{s}}=0 & \text { in } \Omega_{+}, \\ u_{\psi_{s}}=-\psi_{s} \partial_{\nu} u & \text { on } \Gamma, \\ u_{\psi_{s}}=0 & \text { on }\{y=0\} \cap \partial \Omega_{+},\end{cases}
$$

with $u_{\psi_{s}}(-1, y)=u_{\psi_{s}}(1, y)$ for all $y$ such that $( \pm 1, y) \in \overline{\Omega_{+}}$. Now we apply (1.11) and (1.12) to obtain

$$
\frac{d^{2}}{d s^{2}} \mathcal{F}\left(u_{s}\right) \geq\left(C_{0}-C_{1}\|\varphi\|_{C^{2, \alpha}(a, b)}\right)\left\|X_{s} \cdot \nu_{s}\right\|_{H^{1 / 2}\left(\Gamma_{s}\right)}^{2} .
$$

By taking $\|\varphi\|_{C^{2, \alpha}(a, b)} \leq C_{0} /\left(2 C_{1}\right)$ we get $\frac{d^{2}}{d s^{2}} \mathcal{F}\left(u_{s}\right) \geq 0$ for all $s \in[0,1]$. In turn, by (1.11),

$$
\begin{aligned}
\mathcal{F}(u) & =\mathcal{F}\left(u_{1}\right)-\int_{0}^{1}(1-s) \frac{d^{2}}{d s^{2}} \mathcal{F}\left(u_{s}\right) d s \\
& \leq \mathcal{F}\left(u_{1}\right)=\int_{\Phi_{1}\left(\Omega_{+}\right)}\left(\left|\nabla u_{1}\right|^{2}+Q^{2}(\boldsymbol{x})\right) d \boldsymbol{x} .
\end{aligned}
$$

In view of (2.1), $u_{1}$ is the unique minimizer of $\mathcal{F}$ over all functions $v \in H^{1}\left(\Phi_{1}\left(\Omega_{+}\right)\right)$ such that $v=0$ on $\Phi_{1}(\Gamma), v=u$ on $\partial \Phi_{1}\left(\{y=0\} \cap \partial \Omega_{+}\right)$and $v(-1, y)=v(1, y)$ for all $y$ such that $( \pm 1, y) \in \Phi_{1}\left(\overline{\Omega_{+}}\right)$. In particular, for every $v \in \mathcal{A}$ with $\{v>0\}=$ $\Phi_{1}(\{u>0\})$, we have

$$
\mathcal{F}(u) \leq \mathcal{F}(v),
$$

which concludes the proof.

We conclude this section with the proof of the main theorem.

Proof of Theorem 1.1, Let $U \Subset \Omega, \delta>0$, and let $\Phi \in C^{2, \alpha}\left(\mathbb{R}^{2} ; \mathbb{R}^{2}\right)$ be a diffeomorphism satisfying (1.13) and (1.14). 
Step 1: We begin by proving that there exist a constant $C>0$ and an interval $[a, b] \subset(-1,1)$ (independent of $\Phi)$ such that the set $\Phi(\Gamma)$ is the graph of a function $w+\varphi$, where $\varphi \in C^{2, \alpha}(-1,1)$ has compact support in $[a, b]$ and satisfies

$$
\|\varphi\|_{C^{2, \alpha}(-1,1)} \leq C \delta .
$$

Consider the function

$$
\psi(x):=\Phi^{1}(x, w(x)), \quad x \in[-1,1],
$$

where $\Phi=\left(\Phi^{1}, \Phi^{2}\right)$. By the chain rule, $\psi \in C^{2, \alpha}(-1,1)$ with

$$
\begin{aligned}
\psi^{\prime}(x) & =\partial_{x} \Phi^{1}(x, w(x))+w^{\prime}(x) \partial_{y} \Phi^{1}(x, w(x)) \\
& \geq 1-\delta-\delta\left\|w^{\prime}\right\|_{C^{0}(-1,1)} \geq \frac{1}{2}
\end{aligned}
$$

for all $0<\delta<\frac{1}{2+2\left\|w^{\prime}\right\|_{C^{0}(-1,1)}}$, where we used the facts that $\partial_{x} \Phi_{1}(x, y) \geq 1-\delta$ and $\left|\partial_{y} \Phi_{1}(x, y)\right| \leq \delta$ by (1.14). Moreover, by (1.13), $\psi(-1)=-1$ and $\psi(1)=1$. Hence, $\psi:[-1,1] \rightarrow[-1,1]$ is invertible, and by the chain rule $\psi^{-1} \in C^{2, \alpha}(-1,1)$. It follows that

$$
\Phi(\Gamma)=\left\{\left(x, \Phi^{2}\left(\psi^{-1}(x), w\left(\psi^{-1}(x)\right)\right)\right): x \in[-1,1]\right\} .
$$

Define $\varphi(x):=\Phi^{2}\left(\psi^{-1}(x), w\left(\psi^{-1}(x)\right)\right)-w(x)$. By (1.13), $\psi(x)=x$ for $x$ in a neighborhood of -1 and of $1, \Phi^{2}(x, y)=y$ for $(x, y) \notin U$. Hence, $\varphi$ has compact support in $(-1,1)$. A lengthy, but straightforward calculation using (1.14), shows that (4.27) holds.

Step 2: Let now $\left\{\varphi_{n}\right\}_{n}$ be a sequence of polynomials satisfying (3.2) and such that $\varphi_{n} \rightarrow \varphi$ in $C^{2, \alpha}(a, b)$. By Theorems 3.1 and 4.1 for $\delta$ small enough we can construct an admissible flow $\left\{\Phi_{s, n}\right\}_{s \in[0,1]}$ (see Definition 2.1) for every $n$ such that

$$
\Phi_{1, n}(\Gamma)=\left\{\left(x, w(x)+\varphi_{n}(x)\right): x \in(-1,1)\right\}
$$

and

$$
\mathcal{F}(u) \leq \mathcal{F}(v)
$$

for every $v \in \mathcal{A}$ with $\{v>0\}=\Phi_{1, n}(\{u>0\})$.

Consider now a function $v \in \mathcal{A}$ with $\{v>0\}=\Phi(\{u>0\})$, and define

$$
v_{n}(x, y):=v\left(x, y-\varphi_{n}(x)+\varphi(x)\right) .
$$

Then $y<w(x)+\varphi_{n}(x)$ if and only if $y-\varphi_{n}(x)+\varphi(x)<w(x)+\varphi(x)$. Let $\tau>0$. Since $\varphi_{n} \rightarrow \varphi$ in $C^{2, \alpha}(a, b)$, we have that $v_{n} \rightarrow v$ in $H^{1}((-1,1) \times(\tau, \infty))$.

We now construct $\lambda_{\tau} \in C^{\infty}(\mathbb{R})$ such that $0 \leq \lambda_{\tau} \leq 1, \lambda_{\tau}(y)=1$ if $2 \tau \leq y$, $\lambda_{\tau}(y)=0$ if $y \leq \tau$ and $\left|\lambda_{\tau}^{\prime}(y)\right| \leq 2 / \tau$ for all $y \in \mathbb{R}$. Define

$$
v_{n, \tau}(x, y):=\lambda_{\tau}(y) v_{n}(x, y)+\left(1-\lambda_{\tau}(y)\right) u(x, y) .
$$


Since $\Phi_{1, n}$ satisfies (1.13), we have that $v_{n, \tau} \in \mathcal{A}$ and $\left\{v_{n, \tau}>0\right\}=\Phi_{1, n}(\{u>0\})$. Hence, by (4.29), we have

$$
\begin{aligned}
\mathcal{F}(u) & \leq \mathcal{F}\left(v_{n, \tau}\right)=\int_{\Phi_{1, n}\left(\Omega_{+}\right)}\left(\left|\nabla v_{n, \tau}\right|^{2}+Q^{2}(\boldsymbol{x})\right) d \boldsymbol{x} \\
& =\int_{\Omega}\left(\left|\nabla v_{n, \tau}\right|^{2}+\chi_{\left\{v_{n, \tau}>0\right\}} Q^{2}(\boldsymbol{x})\right) d \boldsymbol{x} .
\end{aligned}
$$

Since $\varphi_{n} \rightarrow \varphi$ in $C^{2, \alpha}(a, b)$, if $(x, y) \in \Omega$ is such that $y \neq w(x)+\varphi(x)$ then for all $n$ sufficiently large $y \neq w(x)+\varphi_{n}(x)$, and so $\chi_{\left\{v_{n, \tau}>0\right\}}(x, y)=\chi_{\{v>0\}}(x, y)$. It follows by the Lebesgue dominated convergence theorem that

$$
\lim _{n \rightarrow \infty} \int_{\Omega} \chi_{\left\{v_{n, \tau}>0\right\}} Q^{2}(\boldsymbol{x}) d \boldsymbol{x}=\int_{\Omega} \chi_{\{v>0\}} Q^{2}(\boldsymbol{x}) d \boldsymbol{x} .
$$

On the other hand,

$$
\nabla v_{n, \tau}=\lambda_{\tau} \nabla v_{n}+\left(1-\lambda_{\tau}\right) \nabla u+\left(v_{n}-u\right) \lambda_{\tau}^{\prime} e_{2} .
$$

Hence, using convexity and the inequality $(a+b)^{2} \leq(1+\varepsilon) a^{2}+C_{\varepsilon} b^{2}$, we obtain

$$
\begin{aligned}
\int_{\Omega}\left|\nabla v_{n, \tau}\right|^{2} d \boldsymbol{x} \leq & (1+\varepsilon) \int_{\Omega} \lambda_{\tau}\left|\nabla v_{n}\right|^{2} d \boldsymbol{x}+(1+\varepsilon) \int_{\Omega}\left(1-\lambda_{\tau}\right)|\nabla u|^{2} d \boldsymbol{x} \\
& +\frac{4 C_{\varepsilon}}{\tau^{2}} \int_{(-1,1) \times(\tau, 2 \tau)}\left|v_{n}-u\right|^{2} d \boldsymbol{x} .
\end{aligned}
$$

Since $v_{n} \rightarrow v$ in $H^{1}((-1,1) \times(\tau, \infty))$, letting $n \rightarrow \infty$ we have that

$$
\begin{aligned}
\limsup _{n \rightarrow \infty} \int_{\Omega}\left|\nabla v_{n, \tau}\right|^{2} d \boldsymbol{x} \leq & (1+\varepsilon) \int_{\Omega} \lambda_{\tau}|\nabla v|^{2} d \boldsymbol{x}+(1+\varepsilon) \int_{\Omega}\left(1-\lambda_{\tau}\right)|\nabla u|^{2} d \boldsymbol{x} \\
& +\frac{4 C_{\varepsilon}}{\tau^{2}} \int_{(-1,1) \times(\tau, 2 \tau)}|v-u|^{2} d \boldsymbol{x}
\end{aligned}
$$

By (1.5), if $v$ is of class $C^{1}$, it holds

$$
v(x, y)-u(x, y)=\int_{0}^{y}\left(\partial_{y} v(x, r)-\partial_{y} u(x, r)\right) d r,
$$

and so by Hölder's inequality

$$
\begin{aligned}
\int_{(-1,1) \times(\tau, 2 \tau)}|v-u|^{2} d \boldsymbol{x} & \leq \int_{(-1,1) \times(\tau, 2 \tau)}\left(\int_{0}^{y}\left|\partial_{y} v(x, r)\right|+\left|\partial_{y} u(x, r)\right| d r\right)^{2} d \boldsymbol{x} \\
& \leq \int_{(-1,1) \times(\tau, 2 \tau)} \int_{0}^{y} y\left(\left(\partial_{y} v(x, r)\right)^{2}+\left(\partial_{y} u(x, r)\right)^{2}\right) d r d \boldsymbol{x} \\
& \left.\leq 4 \tau^{2} \int_{(-1,1) \times(0,2 \tau)}\left(\partial_{y} v(x, r)\right)^{2}+\left(\partial_{y} u(x, r)\right)^{2}\right) d x d r
\end{aligned}
$$

By density, the same inequality is satisfied without any extra regularity on $v$.

We now combine (4.30)-4.32) with the previous inequality. By first letting $\tau \rightarrow$ $0^{+}$and then $\varepsilon \rightarrow 0^{+}$, we conclude that $\mathcal{F}(u) \leq \mathcal{F}(v)$. 


\section{Proof of Theorem 1.2}

The proof of Theorem 1.2 is based on some auxiliary lemmas. We start by showing that the first term in the expression (1.12) of the second variation is coercive with respect to the $H^{1 / 2}$ norm of the boundary datum on $\Gamma$.

Lemma 5.1 Let $Q, u$, and $\Gamma$ be as in Theorem 1.2, let $U \subset \Omega$ be an open set such that $U \cap \Gamma \neq \emptyset$, and let $A:=\{u>0\} \cap U$. Assume that $A$ has a Lipschitz boundary. Then there exist two constants $C_{1}, C_{2}>0$, depending on $A$, such that

$$
C_{1}\|\hat{\psi}\|_{H^{1 / 2}(\partial A)}^{2} \leq \inf \left\{\int_{A}|\nabla v|^{2} d \boldsymbol{x}: v \in H^{1}(A), v=\hat{\psi} \text { on } \partial A\right\} \leq C_{2}\|\hat{\psi}\|_{H^{1 / 2}(\partial A)}^{2}
$$

for every $\psi \in C_{c}^{1}(\Gamma \cap U)$, where

$$
\hat{\psi}:= \begin{cases}Q \psi & \text { in } \Gamma \cap U, \\ 0 & \text { in } \partial U \cap\{u>0\} .\end{cases}
$$

Proof. Let $\psi \in C_{c}^{1}(\Gamma \cap U)$. Since $Q^{2} \in C^{0,1}$ and $Q \geq Q_{\min }>0$, we have that $\hat{\psi} \in H^{1 / 2}(\partial A)$, and so there exists $v^{*} \in H^{1}(A)$ such that $v^{*}=\hat{\psi}$ on $\partial A$ in the sense of traces and

$$
\left\|v^{*}\right\|_{H^{1}(A)}^{2} \leq C_{2}\|\hat{\psi}\|_{H^{1 / 2}(\partial A)}^{2},
$$

where $C_{2}$ is a positive constant depending on $A$. Thus, the second inequality in (5.1) holds. On the other hand, the trace operator $T: H^{1}(A) \rightarrow H^{1 / 2}(\partial A)$ is continuous, and so there exists a positive constant $\hat{C}_{1}$, depending on $A$, such that

$$
\|T(v)\|_{H^{1 / 2}(\partial A)}^{2} \leq \hat{C}_{1}\|v\|_{H^{1}(A)}^{2}
$$

for every $v \in H^{1}(A)$. In particular, given $\psi \in C_{c}^{1}(\Gamma \cap U)$, we have that

$$
\|\hat{\psi}\|_{H^{1 / 2}(\partial A)}^{2} \leq \hat{C}_{1}\|v\|_{H^{1}(A)}^{2}
$$

for every $v \in H^{1}(A)$ with $T(v)=\hat{\psi}$. Since $\hat{\psi}=0$ in $\partial U \cap\{u>0\}$, by Poincaré's inequality

$$
\|v\|_{H^{1}(A)}^{2} \leq \tilde{C}_{1}\|\nabla v\|_{L^{2}(A)}^{2}
$$

for every $v \in H^{1}(A)$ with $T(v)=\hat{\psi}$. Combining these two last inequalities, we get the first inequality in (5.1).

Lemma 5.2 Let $Q, u$, and $\Gamma$ be as in Theorem 1.2. For every $\varepsilon>0$ let $U_{\varepsilon}$ be the intersection of $\Omega$ with the $\varepsilon$-tubular neighborhood of $\Gamma$. Define

$$
\mu_{\varepsilon}:=\inf \left\{\int_{U_{\varepsilon} \cap\{u>0\}}\left|\nabla u_{\psi}\right|^{2} d \boldsymbol{x}: \psi \in C_{c}^{1}(\Gamma),\|\psi\|_{L^{2}(\Gamma)}=1\right\},
$$


where for every $\psi \in C_{c}^{1}(\Gamma)$ the function $u_{\psi}$ is the solution to

$$
\begin{cases}\Delta u_{\psi}=0 & \text { in } U_{\varepsilon} \cap\{u>0\} \\ u_{\psi}=Q \psi & \text { on } \Gamma \\ u_{\psi}=0 & \text { on } \partial U_{\varepsilon} \cap\{u>0\},\end{cases}
$$

with $u_{\psi}(-1, y)=u_{\psi}(1, y)$ for all $y$ such that $( \pm 1, y) \in \overline{U_{\varepsilon} \cap\{u>0\}}$. Then

$$
\lim _{\varepsilon \rightarrow 0^{+}} \mu_{\varepsilon}=\infty .
$$

Proof. Assume, by contradiction, that there exist $C>0, \varepsilon_{n} \rightarrow 0^{+}$, and $\psi_{n} \in C_{c}^{1}(\Gamma)$ with $\left\|\psi_{n}\right\|_{L^{2}(\Gamma)}=1$, such that

$$
\int_{U_{\varepsilon_{n}} \cap\{u>0\}}\left|\nabla u_{n}\right|^{2} d \boldsymbol{x} \leq C \quad \text { for every } n,
$$

where $u_{n}:=u_{\psi_{n}}$. We extend $u_{n}$ by 0 to the set $U_{1} \cap\{u>0\}=: V$. Then

$$
\int_{V}\left|\nabla u_{n}\right|^{2} d \boldsymbol{x} \leq C \quad \text { for every } n .
$$

By Poincaré's inequality there exists $u_{\infty} \in H^{1}(V)$ such that $u_{n} \rightarrow u_{\infty}$ weakly in $H^{1}(V)$, up to a subsequence, not relabeled. This implies that $u_{n} \rightarrow u_{\infty}$ strongly in $L^{2}(\partial V)$. Since $\mathcal{L}^{2}\left(U_{\varepsilon_{n}}\right) \rightarrow 0$, we have that $u_{n} \rightarrow 0$ a.e. in $V$, hence $u_{\infty}=0$ and $u_{n} \rightarrow 0$ strongly in $L^{2}(\partial V)$.

On the other hand,

$$
1=\left\|\psi_{n}\right\|_{L^{2}(\Gamma)} \leq C\left\|Q^{-1 / 2}\right\|_{C^{0}}\left\|u_{n}\right\|_{L^{2}(\Gamma)} .
$$

Since the right-hand side tends to 0 , we arrive at a contradiction.

We now prove Theorem 1.2

Proof of Theorem 1.2, By Lemmas 5.1 and 5.2 for $\varepsilon>0$ small enough we have

$\int_{U_{\varepsilon} \cap\{u>0\}} 2\left|\nabla u_{\psi}\right|^{2} d \boldsymbol{x}+\int_{\Gamma}\left(\partial_{\nu} Q^{2}+2 \kappa Q^{2}\right) \psi^{2} d \mathcal{H}^{1} \geq C_{\varepsilon}|\hat{\psi}|_{H^{1 / 2}(\Gamma)}^{2}+\left(\mu_{\varepsilon}-C_{3}\right)\|\psi\|_{L^{2}(\Gamma)}^{2}$,

where $C_{3}:=\left(1+\|\kappa\|_{C^{0}}\right)\left\|Q^{2}\right\|_{C^{0,1}}$. On the other hand,

$$
\begin{aligned}
|\psi|_{H^{1 / 2}(\Gamma)} & =|\hat{\psi} / Q|_{H^{1 / 2}(\Gamma)} \leq\|\hat{\psi}\|_{L^{2}(\Gamma)}|1 / Q|_{C^{0,1}(\Gamma)}+|\hat{\psi}|_{H^{1 / 2}(\Gamma)}\|1 / Q\|_{C^{0}(\Gamma)} \\
& \leq\|\psi\|_{L^{2}(\Gamma)}\|Q\|_{C^{0}(\Gamma)}|1 / Q|_{C^{0,1}(\Gamma)}+|\hat{\psi}|_{H^{1 / 2}(\Gamma)} / Q_{\min },
\end{aligned}
$$

and so

$$
|\hat{\psi}|_{H^{1 / 2}(\Gamma)}^{2} \geq \frac{1}{2} Q_{\mathrm{min}}^{2}|\psi|_{H^{1 / 2}(\Gamma)}^{2}-C_{4}\|\psi\|_{L^{2}(\Gamma)}^{2}
$$


Hence,

$$
\begin{aligned}
\int_{U_{\varepsilon} \cap\{u>0\}} 2\left|\nabla u_{\psi}\right|^{2} d \boldsymbol{x}+\int_{\Gamma}\left(\partial_{\nu} Q^{2}+2 \kappa Q^{2}\right) \psi^{2} d \mathcal{H}^{1} \\
\geq \frac{C_{\varepsilon}}{2} Q_{\min }^{2}|\psi|_{H^{1 / 2}(\Gamma)}^{2}+\left(\mu_{\varepsilon}-C_{3}-\min \left\{C_{\varepsilon} / 2,1\right\} C_{4}\right)\|\psi\|_{L^{2}(\Gamma)}^{2}
\end{aligned}
$$

Since $\mu_{\varepsilon} \rightarrow \infty$ by Lemma 5.2, the inequality (1.15) holds.

The second part of the statement follows from 1.15) by repeating the proof of Theorem 1.1. We omit the details.

\section{Appendix}

Here we sketch the proof of the derivation of the Euler-Lagrange equations (1.3) of (1.1). Let $v \in \mathcal{A}_{0} \cap C^{2}(\bar{\Omega})$ be such that $\partial \Omega_{+} \cap \Omega$ (see (1.7) ) is a manifold of class $C^{2}$, $\mathcal{F}(v) \in \mathbb{R}$ and (1.2) holds. Since $\Omega_{+}$is open, consider variations $\varphi \in C_{c}^{\infty}\left(\Omega_{+}\right)$. For $\varepsilon>0$ sufficiently small it can be shown that $\{v+\varepsilon \varphi>0\}=\{v>0\}$, therefore from (1.2) we obtain

$$
0=\left.\frac{d}{d \varepsilon} \int_{\Omega}|\nabla(v+\varepsilon \varphi)|^{2} d \boldsymbol{x}\right|_{\varepsilon=0}=2 \int_{\Omega_{+}} \nabla v \cdot \nabla \varphi d \boldsymbol{x} .
$$

This gives (1.3) 1 , and the condition $v=0$ on $\Omega \cap \partial\{v>0\}$ follows from the continuity of $v$. To prove that $|\nabla v|=Q$ on $\Omega \cap \partial\{v>0\}$ we use Theorem 2.5 in [AC] to obtain

$$
\lim _{\varepsilon \rightarrow 0^{+}} \int_{\partial\{v>\varepsilon\}}\left(|\nabla v|^{2}-Q^{2}\right) \eta \cdot \nu d \mathcal{H}^{1}=0
$$

for every $\eta \in C_{c}^{\infty}\left(\Omega ; \mathbb{R}^{2}\right)$. Note that in the original proof of $(5.2), v$ was assumed to be a local minimizer, but this property was used only to guarantee the validity of (1.2). In view of the smoothness of $v$ and $\partial \Omega_{+} \cap \Omega$, for $\varepsilon$ sufficiently small $\partial\{v>\varepsilon\}$ is a smooth manifold of class $C^{2}$, and using a partition of unity, it can be shown that (5.2) reduces to

$$
\int_{\partial\{v>0\}}\left(|\nabla v|^{2}-Q^{2}\right) \eta \cdot \nu d \mathcal{H}^{1}=0 .
$$

Extend locally the outward unit normal $\nu$ to $\partial\{v>0\}$ as a $C^{1}$ function $\bar{\nu}$ in an open neighborhood of $\partial\{v>0\}$, and take $\eta:=\varphi \bar{\nu}$, where $\varphi \in C_{c}^{\infty}(\Omega)$ is supported in that neighborhood. Then (5.3) yields

$$
\int_{\partial\{v>0\}}\left(|\nabla v|^{2}-Q^{2}\right) \varphi d \mathcal{H}^{1}=0 .
$$

By the arbitrariness of $\varphi$ we deduce that $|\nabla v|=Q$ on $\Omega \cap \partial\{v>0\}$.

The remaining of the Appendix is dedicated to the proof of Theorem 3.5 . 


\section{Proof of Theorem 3.5, Let}

$$
D:=\{x \in \mathbb{R}: \varphi(x) \neq 0\} .
$$

Step 1: Regularity at points $s \geq 0, x \in D$. By Theorem 3.3 the function $t_{0}$ is of class $C^{\infty}$ in $[0,1] \times D$. Hence, by (3.22) and the smooth dependence of $\xi$ with respect to initial data, we have that $g$ is of class $C^{\infty}$ in $[0,1] \times D$. Taking $t=t_{0}(s, x)$ in (3.16) gives

$$
\int_{0}^{t_{0}(s, x)}\left[\frac{\left(w^{\prime}(\xi(r, x)) \varphi(\xi(r, x))+(\eta(r, x)-w(\xi(r, x))) \varphi^{\prime}(\xi(r, x))\right)^{2}}{\varphi^{2}(\xi(r, x))}+1\right] d r=s
$$

for all $(s, x) \in[0,1] \times D$. Differentiating with respect to $s$, and using (3.24), yields

$$
\partial_{s} t_{0}(s, x)=\frac{1}{1+\left[w^{\prime}\left(\xi\left(t_{0}(s, x), x\right)\right)+s \varphi^{\prime}\left(\xi\left(t_{0}(s, x), x\right)\right)\right]^{2}}
$$

for all $(s, x) \in[0,1] \times D$. Since $t_{0}(0, x)=0$, it follows upon integration and by (3.22) that

$$
t_{0}(s, x)=\int_{0}^{s} \frac{1}{1+\left[w^{\prime}(g(r, x))+r \varphi^{\prime}(g(r, x))\right]^{2}} d r .
$$

By (3.5), (3.22), (3.24), and (5.4),

$$
\begin{aligned}
\partial_{s} g(s, x) & =\partial_{t} \xi\left(t_{0}(s, x), x\right) \partial_{s} t_{0}(s, x) \\
& =\frac{-w^{\prime}(g(s, x)) \varphi(g(s, x))-\left(\eta\left(t_{0}(s, x), x\right)-w(g(s, x))\right) \varphi^{\prime}(g(s, x))}{1+\left[w^{\prime}(g(s, x))+s \varphi^{\prime}(g(s, x))\right]^{2}} \\
& =-\frac{\varphi(g(s, x))\left[w^{\prime}(g(s, x))+s \varphi^{\prime}(g(s, x))\right]}{1+\left[w^{\prime}(g(s, x))+s \varphi^{\prime}(g(s, x))\right]^{2}} .
\end{aligned}
$$

Differentiating (5.6) with respect to $s$ and $x$, respectively, gives

$$
\begin{aligned}
\partial_{s}^{2} g= & -\frac{\varphi^{\prime}(g)\left[w^{\prime}(g)+s \varphi^{\prime}(g)\right] \partial_{s} g+\varphi(g)\left[w^{\prime \prime}(g)+s \varphi^{\prime \prime}(g)\right] \partial_{s} g+\varphi(g) \varphi^{\prime}(g)}{1+\left[w^{\prime}(g)+s \varphi^{\prime}(g)\right]^{2}} \\
& +\frac{2 \varphi(g)\left(w^{\prime}(g)+s \varphi^{\prime}(g)\right)^{2}\left\{\left(w^{\prime \prime}(g)+s \varphi^{\prime \prime}(g)\right) \partial_{s} g+\varphi^{\prime}(g)\right\}}{\left[1+\left(w^{\prime}(g)+s \varphi^{\prime}(g)\right)^{2}\right]^{2}}
\end{aligned}
$$

and

$$
\begin{aligned}
\partial_{x, s}^{2} g= & -\frac{\varphi^{\prime}(g)\left[w^{\prime}(g)+s \varphi^{\prime}(g)\right] \partial_{x} g+\varphi(g)\left[w^{\prime \prime}(g)+s \varphi^{\prime \prime}(g)\right] \partial_{x} g}{1+\left[w^{\prime}(g)+s \varphi^{\prime}(g)\right]^{2}} \\
& +\frac{2 \varphi(g)\left(w^{\prime}(g)+s \varphi^{\prime}(g)\right)^{2}\left(w^{\prime \prime}(g)+s \varphi^{\prime \prime}(g)\right) \partial_{x} g}{\left[1+\left(w^{\prime}(g)+s \varphi^{\prime}(g)\right)^{2}\right]^{2}}
\end{aligned}
$$

while by Schwartz's theorem $\partial_{s, x}^{2} g=\partial_{x, s}^{2} g$. 
On the other hand, by Theorem 3.3, (3.5), (3.20), (3.22), and (3.24), for $(s, x) \in$ $[0,1] \times D$

$$
\begin{aligned}
\partial_{x} g(s, x)= & \partial_{t} \xi\left(t_{0}(s, x), x\right) \partial_{x} t_{0}(s, x)+\partial_{x} \xi\left(t_{0}(s, x), x\right) \\
= & \frac{\partial_{x} \xi\left(t_{0}(s, x), x\right)}{1+\left[w^{\prime}(g(s, x))+s \varphi^{\prime}(g(s, x))\right]^{2}} \\
& +\frac{w^{\prime}(g(s, x))+s \varphi^{\prime}(g(s, x))}{1+\left[w^{\prime}(g(s, x))+s \varphi^{\prime}(g(s, x))\right]^{2}} \partial_{x} \eta\left(t_{0}(s, x), x\right) .
\end{aligned}
$$

Differentiating with respect to $x$, we get

$$
\begin{aligned}
\partial_{x}^{2} g= & -\frac{2\left(w^{\prime}(g)+s \varphi^{\prime}(g)\right)\left(w^{\prime \prime}(g)+s \varphi^{\prime \prime}(g)\right)}{\left[1+\left(w^{\prime}(g)+s \varphi^{\prime}(g)\right)^{2}\right]^{2}} \partial_{x} g \partial_{x} \xi\left(t_{0}, x\right) \\
& +\frac{\partial_{x, t}^{2} \xi\left(t_{0}, x\right) \partial_{x} t_{0}}{1+\left(w^{\prime}(g)+s \varphi^{\prime}(g)\right)^{2}}+\frac{\partial_{x}^{2} \xi\left(t_{0}, x\right)}{1+\left(w^{\prime}(g)+s \varphi^{\prime}(g)\right)^{2}} \\
& +\frac{\left[1-\left(w^{\prime}(g)+s \varphi^{\prime}(g)\right)^{2}\right]\left(w^{\prime \prime}(g)+s \varphi^{\prime \prime}(g)\right)}{\left[1+\left(w^{\prime}(g)+s \varphi^{\prime}(g)\right)^{2}\right]^{2}} \partial_{x} g \partial_{x} \eta\left(t_{0}, x\right) \\
& +\frac{\left(w^{\prime}(g)+s \varphi^{\prime}(g)\right) \partial_{x, t}^{2} \eta\left(t_{0}, x\right) \partial_{x} t_{0}}{1+\left(w^{\prime}(g)+s \varphi^{\prime}(g)\right)^{2}}+\frac{\left(w^{\prime}(g)+s \varphi^{\prime}(g)\right) \partial_{x}^{2} \eta\left(t_{0}, x\right)}{1+\left(w^{\prime}(g)+s \varphi^{\prime}(g)\right)^{2}} \\
= & : I+I I+I I I+I V+V+V I .
\end{aligned}
$$

It remains to study the regularity of $g$ at points $\left(s_{0}, x_{0}\right)$ with $\varphi\left(x_{0}\right)=0$.

Step 2: Regularity at points $s \geq 0, x \notin[a, b]$. Let $s_{0} \geq 0$ and $x_{0} \notin[a, b]$. Since $\varphi \equiv 0$ outside $(a, b)$, by $(3.23)$ we have that $g(s, x)=x$ for all $s \geq 0$ and $x \in \mathbb{R} \backslash(a, b)$. It follows that for all $s \geq 0$ and $x \in \mathbb{R} \backslash[a, b]$,

$$
\begin{aligned}
& \partial_{x} g(s, x)=1, \quad \partial_{s} g(s, x)=0, \\
& \partial_{s}^{2} g(s, x)=\partial_{x}^{2} g(s, x)=\partial_{x, s}^{2} g(s, x)=\partial_{s, x}^{2} g(s, x)=0 .
\end{aligned}
$$

Step 3: Continuity of $g$. Let $s_{0} \geq 0$ and let $x_{0} \in[a, b]$ be such that $\varphi\left(x_{0}\right)=0$. By (3.9) and (3.23), we have that $t_{0}\left(\cdot, x_{0}\right) \equiv 0$ and $g\left(\cdot, x_{0}\right) \equiv x_{0}$, respectively. Then by (3.5), (3.22), and (3.23), we have

$$
\begin{aligned}
g(s, x)-x_{0}= & \xi\left(t_{0}(s, x), x\right)-\xi(0, x)+x-x_{0} \\
= & \int_{0}^{t_{0}(s, x)} \partial_{t} \xi(r, x) d r+x-x_{0} \\
= & -\int_{0}^{t_{0}(s, x)}\left[w^{\prime}(\xi(r, x)) \varphi(\xi(r, x))\right. \\
& +(\eta(r, x)-w(\xi(r, x))) \varphi^{\prime}(\xi(r, x)) d r+x-x_{0} .
\end{aligned}
$$

Since $\xi\left(\cdot, x_{0}\right) \equiv x_{0}$ and $\eta\left(\cdot, x_{0}\right) \equiv w\left(x_{0}\right)$ by (3.6), it follows that

$$
w^{\prime}\left(\xi\left(t, x_{0}\right)\right) \varphi\left(\xi\left(t, x_{0}\right)\right)+\left(\eta\left(t, x_{0}\right)-w\left(\xi\left(t, x_{0}\right)\right)\right) \varphi^{\prime}\left(\xi\left(t, x_{0}\right)\right)=0
$$


for all $t \in[0,1]$. By continuity with respect to initial data, we deduce that the functions $(t, x) \mapsto \xi(t, x)$ and $(t, x) \mapsto \eta(t, x)$ are uniformly continuous on compact sets, and so using also the facts that $w$ is smooth and $\varphi \in C^{2}(\mathbb{R})$, we have that given $\varepsilon>0$ there exists $\delta>0$ such that

$$
\left|w^{\prime}(\xi(t, x)) \varphi(\xi(t, x))+(\eta(t, x)-w(\xi(t, x))) \varphi^{\prime}(\xi(t, x))\right| \leq \varepsilon
$$

for all $t \in[0,1]$ and all $x$ with $\left|x-x_{0}\right| \leq \delta$. Since $0 \leq t_{0} \leq 1$ by (3.11), it follows that

$$
\int_{0}^{t_{0}(s, x)}\left|w^{\prime}(\xi(r, x)) \varphi(\xi(r, x))+(\eta(r, x)-w(\xi(r, x))) \varphi^{\prime}(\xi(r, x))\right| d r \leq \varepsilon
$$

for all $(s, x)$ with $\left|x-x_{0}\right| \leq \delta$. By (5.12) we obtain

$$
\lim _{x \rightarrow x_{0}} g(s, x)=x_{0}
$$

uniformly for all $s \in[0,1]$. This shows that $g$ is continuous at $\left(s_{0}, x_{0}\right)$.

In particular, if $\varphi \neq 0$ in some interval $(\alpha, \beta)$ and $\varphi(\alpha)=\varphi(\beta)=0$, by the continuity of $g$, it follows from (5.5) that

$$
\lim _{(s, x) \rightarrow\left(s_{0}, \alpha\right)^{+}} t_{0}(s, x)=T_{0}\left(s_{0}, \alpha\right),
$$

where

$$
\begin{aligned}
T_{0}(s, x): & =\int_{0}^{s} \frac{1}{1+\left[w^{\prime}(x)+r \varphi^{\prime}(x)\right]^{2}} d r \\
& = \begin{cases}{\left[\arctan \left(w^{\prime}(x)+s \varphi^{\prime}(x)\right)-\arctan \left(w^{\prime}(x)\right)\right] / \varphi^{\prime}(x)} & \text { if } \varphi^{\prime}(x) \neq 0, \\
s /\left[1+\left(w^{\prime}(x)\right)^{2}\right] & \text { if } \varphi^{\prime}(x)=0 .\end{cases}
\end{aligned}
$$

Since $t_{0}(s, \alpha)=0$, this shows that the function $t_{0}$ is discontinuous at $\left(s_{0}, \alpha\right)$ for all $s_{0}>0$. A similar result holds at the endpoint $\beta$.

Step 4: Existence and continuity of $\partial_{s} g$ and $\partial_{x} g$. Let $s_{0} \geq 0$ and let $x_{0} \in[a, b]$ be such that $\varphi\left(x_{0}\right)=0$. By (3.23), we have that $g\left(\cdot, x_{0}\right) \equiv x_{0}$, and so

$$
\partial_{s} g\left(s, x_{0}\right)=0
$$

for all $s \geq 0$. On the other hand, if $\varphi \neq 0$ in some interval $\left(x_{0}, x_{0}+\delta\right)$ (the case $\left(x_{0}-\delta, x_{0}\right)$ is similar), by the continuity of $g$ and (5.6),

$$
\partial_{s} g(s, x) \rightarrow \frac{-\varphi\left(x_{0}\right)\left[w^{\prime}\left(x_{0}\right)+s \varphi^{\prime}\left(x_{0}\right)\right]}{1+\left[w^{\prime}\left(x_{0}\right)+s \varphi^{\prime}\left(x_{0}\right)\right]^{2}}=0
$$

as $(s, x) \rightarrow\left(s_{0}, x_{0}\right)^{+}$. Hence, $\partial_{s} g$ is continuous at $\left(s_{0}, x_{0}\right)$ for all $s_{0} \geq 0$.

Next, we prove the existence and continuity of $\partial_{x} g$ at $\left(s_{0}, x_{0}\right)$ for all $s_{0} \geq 0$. We assume, as before, that $\varphi \neq 0$ in some interval $\left(x_{0}, x_{0}+\delta\right)$ (the case $\left(x_{0}-\delta, x_{0}\right)$ is similar). Differentiating (3.5) with respect to $x$, we obtain

$$
\left\{\begin{array}{l}
\partial_{t}\left(\partial_{x} \xi\right)=-\left[w^{\prime \prime}(\xi) \varphi(\xi)+(\eta-w(\xi)) \varphi^{\prime \prime}(\xi)\right] \partial_{x} \xi-\varphi^{\prime}(\xi) \partial_{x} \eta \\
\partial_{t}\left(\partial_{x} \eta\right)=\varphi^{\prime}(\xi) \partial_{x} \xi \\
\partial_{x} \xi(0, x)=1 \\
\partial_{x} \eta(0, x)=w^{\prime}(x) .
\end{array}\right.
$$


Since $\xi\left(\cdot, x_{0}\right) \equiv x_{0}$ and $\eta\left(\cdot, x_{0}\right) \equiv w\left(x_{0}\right)$ by (3.6), we have that $\partial_{x} \xi\left(\cdot, x_{0}\right)$ and $\partial_{x} \eta\left(\cdot, x_{0}\right)$ solve the system

$$
\left\{\begin{array}{l}
\partial_{t}\left(\partial_{x} \xi\left(\cdot, x_{0}\right)\right)=-\varphi^{\prime}\left(x_{0}\right) \partial_{x} \eta\left(\cdot, x_{0}\right) \\
\partial_{t}\left(\partial_{x} \eta\left(\cdot, x_{0}\right)\right)=\varphi^{\prime}\left(x_{0}\right) \partial_{x} \xi\left(\cdot, x_{0}\right) \\
\partial_{x} \xi\left(0, x_{0}\right)=1 \\
\partial_{x} \eta\left(0, x_{0}\right)=w^{\prime}\left(x_{0}\right)
\end{array}\right.
$$

and so

$$
\begin{aligned}
& \partial_{x} \xi\left(t, x_{0}\right)=\cos \left(\varphi^{\prime}\left(x_{0}\right) t\right)-w^{\prime}\left(x_{0}\right) \sin \left(\varphi^{\prime}\left(x_{0}\right) t\right), \\
& \partial_{x} \eta\left(t, x_{0}\right)=w^{\prime}\left(x_{0}\right) \cos \left(\varphi^{\prime}\left(x_{0}\right) t\right)+\sin \left(\varphi^{\prime}\left(x_{0}\right) t\right) .
\end{aligned}
$$

By the the continuity of $\partial_{x} \xi$ and $\partial_{x} \eta$, (5.14) and (5.18),

$$
\begin{aligned}
\lim _{(s, x) \rightarrow\left(s_{0}, x_{0}\right)^{+}} \partial_{x} \xi\left(t_{0}(s, x), x\right)=\partial_{x} \xi\left(T_{0}\left(s_{0}, x_{0}\right), x_{0}\right) \\
=\cos \left(\varphi^{\prime}\left(x_{0}\right) T_{0}\left(s, x_{0}\right)\right)-w^{\prime}\left(x_{0}\right) \sin \left(\varphi^{\prime}\left(x_{0}\right) T_{0}\left(s, x_{0}\right)\right) \\
=\frac{\sqrt{1+\left[w^{\prime}\left(x_{0}\right)\right]^{2}}}{\sqrt{1+\left[w^{\prime}\left(x_{0}\right)+s_{0} \varphi^{\prime}\left(x_{0}\right)\right]^{2}}}
\end{aligned}
$$

and

$$
\begin{aligned}
\lim _{(s, x) \rightarrow\left(s_{0}, x_{0}\right)^{+}} \partial_{x} \eta\left(t_{0}(s, x), x\right)=\partial_{x} \eta\left(T_{0}\left(s_{0}, x_{0}\right), x_{0}\right) \\
=w^{\prime}\left(x_{0}\right) \cos \left(\varphi^{\prime}\left(x_{0}\right) T_{0}\left(s, x_{0}\right)\right)+\sin \left(\varphi^{\prime}\left(x_{0}\right) T_{0}\left(s, x_{0}\right)\right) \\
=\frac{\left(w^{\prime}\left(x_{0}\right)+s_{0} \varphi^{\prime}\left(x_{0}\right)\right) \sqrt{1+\left[w^{\prime}\left(x_{0}\right)\right]^{2}}}{\sqrt{1+\left[w^{\prime}\left(x_{0}\right)+s_{0} \varphi^{\prime}\left(x_{0}\right)\right]^{2}}}
\end{aligned}
$$

where we have used the formulas

$$
\begin{aligned}
\cos (\arctan (x+y)-\arctan y) & =\frac{1+x y+y^{2}}{\sqrt{1+y^{2}} \sqrt{1+(x+y)^{2}}}, \\
\sin (\arctan (x+y)-\arctan y) & =\frac{x}{\sqrt{1+y^{2}} \sqrt{1+(x+y)^{2}}} .
\end{aligned}
$$

Note that

$$
\left[w^{\prime}\left(x_{0}\right)+s \varphi^{\prime}\left(x_{0}\right)\right] \partial_{x} \xi\left(T_{0}\left(s, x_{0}\right), x_{0}\right)-\partial_{x} \eta\left(T_{0}\left(s, x_{0}\right), x_{0}\right)=0
$$

for every $s \in[0,1]$.

By (5.9), (5.19), (5.20), we obtain

$$
\lim _{(s, x) \rightarrow\left(s_{0}, x_{0}\right)^{+}} \partial_{x} g(s, x)=\frac{\sqrt{1+\left[w^{\prime}\left(x_{0}\right)\right]^{2}}}{\sqrt{1+\left[w^{\prime}\left(x_{0}\right)+s_{0} \varphi^{\prime}\left(x_{0}\right)\right]^{2}}} .
$$


By the continuity of $g$ proved in Step 3,

$$
\lim _{x \rightarrow x_{0}^{+}} \frac{g\left(s_{0}, x\right)-g\left(s_{0}, x_{0}\right)}{x-x_{0}}=\frac{0}{0},
$$

and so we can apply L'Hôpital's rule to the function $x \mapsto g\left(s_{0}, x\right)$ to conclude that there exists

$$
\lim _{x \rightarrow x_{0}^{+}} \frac{g\left(s_{0}, x\right)-g\left(s_{0}, x_{0}\right)}{x-x_{0}}=\lim _{x \rightarrow x_{0}^{+}} \frac{\partial_{x} g\left(s_{0}, x\right)}{1}=\frac{\sqrt{1+\left[w^{\prime}\left(x_{0}\right)\right]^{2}}}{\sqrt{1+\left[w^{\prime}\left(x_{0}\right)+s_{0} \varphi^{\prime}\left(x_{0}\right)\right]^{2}}} .
$$

If $\varphi \neq 0$ also in some interval $\left(x_{0}-\delta_{1}, x_{0}\right)$, then we conclude in the same way that

$$
\lim _{x \rightarrow x_{0}^{-}} \frac{g\left(s_{0}, x\right)-g\left(s_{0}, x_{0}\right)}{x-x_{0}}=\lim _{x \rightarrow x_{0}^{-}} \frac{\partial_{x} g\left(s_{0}, x\right)}{1}=\frac{\sqrt{1+\left[w^{\prime}\left(x_{0}\right)\right]^{2}}}{\sqrt{1+\left[w^{\prime}\left(x_{0}\right)+s_{0} \varphi^{\prime}\left(x_{0}\right)\right]^{2}}},
$$

and so we deduce that there exists

$$
\partial_{x} g\left(s_{0}, x_{0}\right)=\frac{\sqrt{1+\left[w^{\prime}\left(x_{0}\right)\right]^{2}}}{\sqrt{1+\left[w^{\prime}\left(x_{0}\right)+s_{0} \varphi^{\prime}\left(x_{0}\right)\right]^{2}}}
$$

and that $\partial_{x} g$ is continuous at $\left(s_{0}, x_{0}\right)$. On the other hand, if $\varphi=0$ in some interval $\left(x_{0}-\delta_{1}, x_{0}\right)$, then $x_{0}=a$, and $\varphi^{\prime}\left(x_{0}\right)=0$. It follows that the limit in (5.22) is 1 , and so by (5.11) we obtain again that there exists $\partial_{x} g\left(s_{0}, x_{0}\right)=1$ and that $\partial_{x} g$ is continuous at $\left(s_{0}, x_{0}\right)$.

Step 5: Existence and continuity of $\partial_{s}^{2} g, \partial_{x, s}^{2} g$, and $\partial_{s, x}^{2} g$. Let $s_{0} \geq 0$ and let $x_{0} \in[a, b]$ be such that $\varphi\left(x_{0}\right)=0$. We assume, as before, that $\varphi \neq 0$ in some interval $\left(x_{0}, x_{0}+\delta\right)$ (the case $\left(x_{0}-\delta, x_{0}\right)$ is similar). By (5.16), we have that $\partial_{s}^{2} g\left(s, x_{0}\right)=0$ for all $s \geq 0$. On the other hand, by (5.7) and the continuity of $g$ and $\partial_{s} g$,

$$
\lim _{(s, x) \rightarrow\left(s_{0}, a\right)^{+}} \partial_{s}^{2} g(s, x)=0 .
$$

By (5.8) and the continuity of $g, \partial_{s} g$, and $\partial_{x} g$,

$$
\lim _{(s, x) \rightarrow\left(s_{0}, x_{0}\right)^{+}} \partial_{x, s}^{2} g(s, x)=-\frac{\varphi^{\prime}\left(x_{0}\right)\left[w^{\prime}\left(x_{0}\right)+s_{0} \varphi^{\prime}\left(x_{0}\right)\right] \partial_{x} g\left(s_{0}, x_{0}\right)}{1+\left[w^{\prime}\left(x_{0}\right)+s \varphi^{\prime}\left(x_{0}\right)\right]^{2}} .
$$

On the other hand, by Step 4,

$$
\lim _{x \rightarrow x_{0}^{+}} \frac{\partial_{s} g\left(s_{0}, x\right)-\partial_{s} g\left(s_{0}, x_{0}\right)}{x-x_{0}}=\frac{0}{0},
$$

and so we can apply L'Hôpital's rule to the function $x \mapsto \partial_{s} g\left(s_{0}, x\right)$ to conclude that there exists

$$
\begin{aligned}
\lim _{x \rightarrow x_{0}^{+}} \frac{\partial_{s} g\left(s_{0}, x\right)-\partial_{s} g\left(s_{0}, x_{0}\right)}{x-x_{0}} & =\lim _{x \rightarrow x_{0}^{+}} \frac{\partial_{x, s}^{2} g\left(s_{0}, x\right)}{1} \\
& =-\frac{\varphi^{\prime}\left(x_{0}\right)\left[w^{\prime}\left(x_{0}\right)+s_{0} \varphi^{\prime}\left(x_{0}\right)\right] \partial_{x} g\left(s_{0}, x_{0}\right)}{1+\left[w^{\prime}\left(x_{0}\right)+s \varphi^{\prime}\left(x_{0}\right)\right]^{2}} .
\end{aligned}
$$


If $\varphi \neq 0$ also in some interval $\left(x_{0}-\delta_{1}, x_{0}\right)$, then we deduce as in the previous step that that there exists $\partial_{x, s}^{2} g\left(s_{0}, x_{0}\right)$ and that $\partial_{x, s}^{2} g$ is continuous at $\left(s_{0}, x_{0}\right)$. On the other hand, if $\varphi=0$ in some interval $\left(x_{0}-\delta_{1}, x_{0}\right)$, then $x_{0}=a$, and $\varphi^{\prime}\left(x_{0}\right)=0$. It follows from (5.24) and (5.11) we obtain again that there exists $\partial_{x, s}^{2} g\left(s_{0}, x_{0}\right)=0$ and that $\partial_{x, s}^{2} g$ is continuous at $\left(s_{0}, x_{0}\right)$.

In both cases we can apply Schwartz's theorem to conclude that there exists $\partial_{s, x}^{2} g\left(s_{0}, x_{0}\right)$ and that

$$
\partial_{s, x}^{2} g\left(s_{0}, x_{0}\right)=\partial_{x, s}^{2} g\left(s_{0}, x_{0}\right) .
$$

Step 6: Existence and continuity of $\partial_{x}^{2} g$. By Step 3, (5.19), (5.20), (5.22), and (5.10), we have

$$
\begin{aligned}
\lim _{(s, x) \rightarrow\left(s_{0}, x_{0}\right)^{+}} I= & -\frac{2\left(w^{\prime}\left(x_{0}\right)+s \varphi^{\prime}\left(x_{0}\right)\right)\left(w^{\prime \prime}\left(x_{0}\right)+s \varphi^{\prime \prime}\left(x_{0}\right)\right)}{\left[1+\left(w^{\prime}\left(x_{0}\right)+s \varphi^{\prime}\left(x_{0}\right)\right)^{2}\right]^{2}} \\
& \times \partial_{x} g\left(s_{0}, x_{0}\right) \partial_{x} \xi\left(T_{0}\left(s_{0}, x_{0}\right), x_{0}\right), \\
\lim _{(s, x) \rightarrow\left(s_{0}, x_{0}\right)^{+}} I V= & \frac{\left[1-\left(w^{\prime}\left(x_{0}\right)+s \varphi^{\prime}\left(x_{0}\right)\right)^{2}\right]\left(w^{\prime \prime}\left(x_{0}\right)+s \varphi^{\prime \prime}\left(x_{0}\right)\right)}{\left[1+\left(w^{\prime}\left(x_{0}\right)+s \varphi^{\prime}\left(x_{0}\right)\right)^{2}\right]^{2}} \\
& \times \partial_{x} g\left(s_{0}, x_{0}\right) \partial_{x} \eta\left(T_{0}\left(s_{0}, x_{0}\right), x_{0}\right) .
\end{aligned}
$$

On the other hand, by Step 3, the continuity of $\partial_{x}^{2} \xi$ and $\partial_{x}^{2} \eta$, (5.10), and (5.14),

$$
\lim _{(s, x) \rightarrow\left(s_{0}, x_{0}\right)^{+}} I I I=\frac{\partial_{x}^{2} \xi\left(T_{0}\left(s_{0}, x_{0}\right), x_{0}\right)}{1+\left(w^{\prime}\left(x_{0}\right)+s_{0} \varphi^{\prime}\left(x_{0}\right)\right)^{2}},
$$

and

$$
\lim _{(s, x) \rightarrow\left(s_{0}, x_{0}\right)^{+}} V I=\frac{\left(w^{\prime}\left(x_{0}\right)+s \varphi^{\prime}\left(x_{0}\right)\right) \partial_{x}^{2} \eta\left(T_{0}\left(s_{0}, x_{0}\right), x_{0}\right)}{1+\left(w^{\prime}\left(x_{0}\right)+s \varphi^{\prime}\left(x_{0}\right)\right)^{2}} .
$$

It remains to estimate $I I$ and $V$ in (5.10). By Taylor's formula, we obtain

$$
w^{\prime}(z)+s \varphi^{\prime}(z)=w^{\prime}\left(x_{0}\right)+s \varphi^{\prime}\left(x_{0}\right)+\left(w^{\prime \prime}\left(x_{0}\right)+s \varphi^{\prime \prime}\left(x_{0}\right)\right)\left(z-x_{0}\right)+o\left(z-x_{0}\right) .
$$

Hence, also by (5.13),

$$
\begin{aligned}
& \frac{\left[w^{\prime}(g(s, x))+s \varphi^{\prime}(g(s, x))\right] \partial_{x} \xi\left(t_{0}(s, x), x\right)-\partial_{x} \eta\left(t_{0}(s, x), x\right)}{g(s, x)-x_{0}} \\
& =\frac{\left(w^{\prime}\left(x_{0}\right)+s \varphi^{\prime}\left(x_{0}\right)\right) \partial_{x} \xi\left(t_{0}(s, x), x\right)-\partial_{x} \eta\left(t_{0}(s, x), x\right)}{g(s, x)-x_{0}} \\
& \quad+\left(w^{\prime \prime}\left(x_{0}\right)+s \varphi^{\prime \prime}\left(x_{0}\right)+o(1)\right) \partial_{x} \xi\left(t_{0}(s, x), x\right) .
\end{aligned}
$$


By repeated applications of the mean value theorem, we have that

$$
\begin{aligned}
\left(w^{\prime}\left(x_{0}\right)\right. & \left.+s \varphi^{\prime}\left(x_{0}\right)\right) \partial_{x} \xi\left(t_{0}(s, x), x\right)-\partial_{x} \eta\left(t_{0}(s, x), x\right) \\
= & \left(w^{\prime}\left(x_{0}\right)+s \varphi^{\prime}\left(x_{0}\right)\right) \partial_{x} \xi\left(t_{0}(s, x), x_{0}\right)-\partial_{x} \eta\left(t_{0}(s, x), x_{0}\right) \\
& +\left(x-x_{0}\right)\left[\left(w^{\prime}\left(x_{0}\right)+s \varphi^{\prime}\left(x_{0}\right)\right) \partial_{x}^{2} \xi\left(t_{0}(s, x), x_{1}\right)-\partial_{x}^{2} \eta\left(t_{0}(s, x), x_{1}\right)\right] \\
= & \left(x-x_{0}\right)\left\{( \frac { t _ { 0 } ( s , x ) - T _ { 0 } ( s , x _ { 0 } ) } { x - x _ { 0 } } ) \left[\left(w^{\prime}\left(x_{0}\right)+s \varphi^{\prime}\left(x_{0}\right)\right) \partial_{x, t}^{2} \xi\left(t_{1}, x_{0}\right)\right.\right. \\
& \left.\left.-\partial_{x, t}^{2} \eta\left(t_{1}, x_{0}\right)\right]+\left[\left(w^{\prime}\left(x_{0}\right)+s \varphi^{\prime}\left(x_{0}\right)\right) \partial_{x}^{2} \xi\left(t_{0}(s, x), x_{1}\right)-\partial_{x}^{2} \eta\left(t_{0}(s, x), x_{1}\right)\right]\right\}
\end{aligned}
$$

for some $x_{1}$ between $x$ and $x_{0}$ and for some $t_{1}(s, x)$ between $t_{0}(s, x)$ and $T_{0}\left(s, x_{0}\right)$, and where we have used (5.21).

By (3.23), (5.5), (5.15), and again the mean value theorem, we get

$$
\begin{aligned}
& t_{0}(s, x)-T_{0}\left(s, x_{0}\right) \\
& =\int_{0}^{s} \frac{\left(w^{\prime}\left(x_{0}\right)+r \varphi^{\prime}\left(x_{0}\right)\right)^{2}-\left(w^{\prime}(g(r, x))+r \varphi^{\prime}(g(r, x))\right)^{2}}{\left[1+\left(w^{\prime}(g(r, x))+r \varphi^{\prime}(g(r, x))\right)^{2}\right]\left[1+\left(w^{\prime}\left(x_{0}\right)+r \varphi^{\prime}\left(x_{0}\right)\right)^{2}\right]} d r \\
& =-\left(x-x_{0}\right) \int_{0}^{s} \frac{2\left(w^{\prime}(g(r, c))+r \varphi^{\prime}(g(r, c))\right)\left(w^{\prime \prime}(g(r, c))+r \varphi^{\prime \prime}(g(r, c))\right) \partial_{x} g(r, c)}{\left[1+\left(w^{\prime}(g(r, x))+r \varphi^{\prime}(g(r, x))\right)^{2}\right]\left[1+\left(w^{\prime}\left(x_{0}\right)+r \varphi^{\prime}\left(x_{0}\right)\right)^{2}\right]} d r
\end{aligned}
$$

for some $c=c\left(r, x, x_{0}\right)$ between $x$ and $x_{0}$. Hence, by (3.23) and the continuity of $g$ and $\partial_{x} g$,

$$
\begin{aligned}
& \lim _{(s, x) \rightarrow\left(s_{0}, x_{0}\right)^{+}} \frac{t_{0}(s, x)-T_{0}\left(s, x_{0}\right)}{x-x_{0}} \\
& =-\int_{0}^{s_{0}} \frac{2\left(w^{\prime}\left(x_{0}\right)+r \varphi^{\prime}\left(x_{0}\right)\right)\left(w^{\prime \prime}\left(x_{0}\right)+r \varphi^{\prime \prime}\left(x_{0}\right)\right) \partial_{x} g\left(r, x_{0}\right)}{\left[1+\left(w^{\prime}\left(x_{0}\right)+r \varphi^{\prime}\left(x_{0}\right)\right)^{2}\right]^{2}} d r=: \ell_{1} .
\end{aligned}
$$

By (3.23), (5.22), and the mean value theorem, we deduce that

$$
\frac{g(s, x)-x_{0}}{x-x_{0}}=\frac{g(s, x)-g\left(s, x_{0}\right)}{x-x_{0}}=\partial_{x} g(s, \theta) \rightarrow \partial_{x} g\left(s_{0}, x_{0}\right)
$$

as $(s, x) \rightarrow\left(s_{0}, x_{0}\right)$. Hence, letting $(s, x) \rightarrow\left(s_{0}, x_{0}\right)$ in (5.29) and using (5.30), (5.32), and (5.33) gives

$$
\begin{aligned}
& \lim _{(s, x) \rightarrow\left(s_{0}, x_{0}\right)^{+}} \frac{\left[w^{\prime}(g(s, x))+s \varphi^{\prime}(g(s, x))\right] \partial_{x} \xi\left(t_{0}(s, x), x\right)-\partial_{x} \eta\left(t_{0}(s, x), x\right)}{g(s, x)-x_{0}} \\
& =\frac{\left\{\ell_{1}\left[\left(w^{\prime}\left(x_{0}\right)+s \varphi^{\prime}\left(x_{0}\right)\right) \partial_{x, t}^{2} \xi\left(T_{0}\left(s_{0}, x_{0}\right), x_{0}\right)-\partial_{x, t}^{2} \eta\left(T_{0}\left(s_{0}, x_{0}\right), x_{0}\right)\right]\right.}{\partial_{x} g\left(s_{0}, x_{0}\right)} \\
& \left.\quad+\left[\left(w^{\prime}\left(x_{0}\right)+s \varphi^{\prime}\left(x_{0}\right)\right) \partial_{x}^{2} \xi\left(T_{0}\left(s_{0}, x_{0}\right), x_{0}\right)-\partial_{x}^{2} \eta\left(T_{0}\left(s_{0}, x_{0}\right), x_{0}\right)\right]\right\} \\
& \quad+\left(w^{\prime \prime}\left(x_{0}\right)+s \varphi^{\prime \prime}\left(x_{0}\right)\right) \partial_{x} \xi\left(T_{0}\left(s_{0}, x_{0}\right), x_{0}\right)=: \ell_{2} .
\end{aligned}
$$


By (3.24), (3.20), (5.17), (5.10),

$$
\begin{aligned}
I I= & -\frac{\left[w^{\prime \prime}(g) \varphi(g)+s \varphi(g) \varphi^{\prime \prime}(g)\right] \partial_{x} \xi\left(t_{0}, x\right)-\varphi^{\prime}(g) \partial_{x} \eta\left(t_{0}, x\right)}{1+\left(w^{\prime}(g)+s \varphi^{\prime}(g)\right)^{2}} \\
\times & \frac{\left[w^{\prime}(g)+s \varphi^{\prime}(g)\right] \partial_{x} \xi\left(t_{0}, x\right)-\partial_{x} \eta\left(t_{0}, x\right)}{\varphi(g)\left[1+\left(w^{\prime}(g)+s \varphi^{\prime}(g)\right)^{2}\right]} \\
= & -\left[w^{\prime \prime}(g)+s \varphi^{\prime \prime}(g)\right] \partial_{x} \xi\left(t_{0}, x\right) \frac{\left[w^{\prime}(g)+s \varphi^{\prime}(g)\right] \partial_{x} \xi\left(t_{0}, x\right)-\partial_{x} \eta\left(t_{0}, x\right)}{\left[1+\left(w^{\prime}(g)+s \varphi^{\prime}(g)\right)^{2}\right]^{2}} \\
& -\frac{\varphi^{\prime}(g)}{\varphi(g)} \partial_{x} \eta\left(t_{0}, x\right) \frac{\left[w^{\prime}(g)+s \varphi^{\prime}(g)\right] \partial_{x} \xi\left(t_{0}, x\right)-\partial_{x} \eta\left(t_{0}, x\right)}{\left[1+\left(w^{\prime}(g)+s \varphi^{\prime}(g)\right)^{2}\right]^{2}} \\
= & I I_{a}+I I_{b} .
\end{aligned}
$$

By Step 3, (5.19), (5.20), and (5.21),

$$
\begin{aligned}
& \lim _{(s, x) \rightarrow\left(s_{0}, x_{0}\right)^{+}} I I_{a}=-\left[w^{\prime \prime}\left(x_{0}\right)+s_{0} \varphi^{\prime \prime}\left(x_{0}\right)\right] \partial_{x} \xi\left(T_{0}\left(s_{0}, x_{0}\right), x_{0}\right) \\
& \times \frac{\left[w^{\prime}\left(x_{0}\right)+s_{0} \varphi^{\prime}\left(x_{0}\right)\right] \partial_{x} \xi\left(T_{0}\left(s_{0}, x_{0}\right), x_{0}\right)-\partial_{x} \eta\left(T_{0}\left(s_{0}, x_{0}\right), x_{0}\right)}{\left[1+\left(w^{\prime}\left(x_{0}\right)+s_{0} \varphi^{\prime}\left(x_{0}\right)\right)^{2}\right]^{2}}=0 .
\end{aligned}
$$

Since $\varphi$ is a polynomial with $\varphi\left(x_{0}\right)=0$, we may write

$$
\varphi(z)=p(z)\left(z-x_{0}\right)^{k},
$$

where $p$ is a polynomial with $p\left(x_{0}\right) \neq 0$ and $k \geq 1$. In turn,

$$
\varphi^{\prime}(z)=\left(z-x_{0}\right)^{k-1}\left[p^{\prime}(z)\left(z-x_{0}\right)+k p(z)\right] .
$$

By (5.37), (5.38),

$$
\begin{aligned}
I I_{b}= & -\frac{\left[p^{\prime}(g)\left(g-x_{0}\right)+k p(g)\right] \partial_{x} \eta\left(t_{0}, x\right)}{\left[1+\left(w^{\prime}(g)+s \varphi^{\prime}(g)\right)^{2}\right]^{2} p(g)} \\
& \times \frac{\left[w^{\prime}(g)+s \varphi^{\prime}(g)\right] \partial_{x} \xi\left(t_{0}, x\right)-\partial_{x} \eta\left(t_{0}, x\right)}{g-x_{0}},
\end{aligned}
$$

and so by Step 3, (3.2), (5.20), and (5.34),

$$
\lim _{(s, x) \rightarrow\left(s_{0}, x_{0}\right)^{+}} I I_{b}=-\frac{k \partial_{x} \eta\left(T_{0}\left(s_{0}, x_{0}\right), x_{0}\right)}{\left[1+\left(w^{\prime}\left(x_{0}\right)+s_{0} \varphi^{\prime}\left(x_{0}\right)\right)^{2}\right]^{2}} \ell_{2} .
$$


By (3.24), (5.37), (3.20), (5.17), (5.10), (5.38),

$$
\begin{aligned}
V= & \frac{\left(w^{\prime}(g)+s \varphi^{\prime}(g)\right) \varphi^{\prime}(g) \partial_{x} \xi\left(t_{0}, x\right)}{1+\left(w^{\prime}(g)+s \varphi^{\prime}(g)\right)^{2}} \frac{\left[w^{\prime}(g)+s \varphi^{\prime}(g)\right] \partial_{x} \xi\left(t_{0}, x\right)-\partial_{x} \eta\left(t_{0}, x\right)}{\varphi(g)\left[1+\left(w^{\prime}(g)+s \varphi^{\prime}(g)\right)^{2}\right]} \\
= & \frac{\left(w^{\prime}(g)+s \varphi^{\prime}(g)\right)\left[p^{\prime}(g)\left(g-x_{0}\right)+k p(g)\right] \partial_{x} \xi\left(t_{0}, x\right)}{\left[1+\left(w^{\prime}(g)+s \varphi^{\prime}(g)\right)^{2}\right]^{2} p(g)} \\
& \times \frac{\left[w^{\prime}(g)+s \varphi^{\prime}(g)\right] \partial_{x} \xi\left(t_{0}, x\right)-\partial_{x} \eta\left(t_{0}, x\right)}{g-x_{0}},
\end{aligned}
$$

and so by Step 3, (5.20), and (5.34),

$$
\lim _{(s, x) \rightarrow\left(s_{0}, x_{0}\right)^{+}} V=\frac{\left(w^{\prime}\left(x_{0}\right)+s \varphi^{\prime}\left(x_{0}\right)\right) k \partial_{x} \xi\left(T_{0}\left(s_{0}, x_{0}\right), x_{0}\right)}{\left[1+\left(w^{\prime}\left(x_{0}\right)+s_{0} \varphi^{\prime}\left(x_{0}\right)\right)^{2}\right]^{2}} \ell_{2} .
$$

Finally, by (5.10), (5.25), (5.26), (5.35), (5.36), (5.39), (5.40), (5.27), and (5.28), there exists

$$
\lim _{(s, x) \rightarrow\left(s_{0}, x_{0}\right)^{+}} \partial_{x}^{2} g(s, x)=\ell_{3} \in \mathbb{R} .
$$

By the continuity of $\partial_{x} g$ proved in Step 4 ,

$$
\lim _{x \rightarrow x_{0}^{+}} \frac{\partial_{x} g\left(s_{0}, x\right)-\partial_{x} g\left(s_{0}, x_{0}\right)}{x-x_{0}}=\frac{0}{0},
$$

and so we can apply L'Hôpital's rule to the function $x \mapsto \partial_{x} g\left(s_{0}, x\right)$ to conclude that there exists

$$
\lim _{x \rightarrow x_{0}^{+}} \frac{\partial_{x} g\left(s_{0}, x\right)-\partial_{x} g\left(s_{0}, x_{0}\right)}{x-x_{0}}=\lim _{x \rightarrow x_{0}^{+}} \frac{\partial_{x}^{2} g\left(s_{0}, x\right)}{1}=\ell_{3} .
$$

If $\varphi \neq 0$ also in some interval $\left(x_{0}-\delta_{1}, x_{0}\right)$, then the limit as $x \rightarrow x_{0}^{-}$is still $\ell_{3}$, and so there exists $\partial_{x}^{2} g\left(s_{0}, x_{0}\right)=\ell_{3}$ and $\partial_{x}^{2} g$ is continuous at $\left(s_{0}, x_{0}\right)$. On the other hand, if $\varphi=0$ in some interval $\left(x_{0}-\delta_{1}, x_{0}\right)$, then $x_{0}=a$, and $\varphi^{\prime}\left(x_{0}\right)=\varphi^{\prime \prime}\left(x_{0}\right)=0$.

Then by (5.19), (5.20), (5.22),

$$
\begin{aligned}
\partial_{x} \xi\left(T_{0}\left(s_{0}, x_{0}\right), x_{0}\right) & =1, \quad \partial_{x} \eta\left(T_{0}\left(s_{0}, x_{0}\right), x_{0}\right)=w^{\prime}\left(x_{0}\right), \\
\partial_{x} g\left(s_{0}, x_{0}\right) & =1 .
\end{aligned}
$$

To calculate $\partial_{x}^{2} \xi\left(T_{0}\left(s_{0}, x_{0}\right), x_{0}\right)$ and $\partial_{x}^{2} \eta\left(T_{0}\left(s_{0}, x_{0}\right), x_{0}\right)$, differentiate (5.17) with respect to $x$ to obtain

$$
\left\{\begin{array}{l}
\partial_{t}\left(\partial_{x}^{2} \xi\right)=-\left[w^{\prime \prime \prime}(\xi) \varphi(\xi)+w^{\prime \prime}(\xi) \varphi^{\prime}(\xi)-w^{\prime}(\xi) \varphi^{\prime \prime}(\xi)\right. \\
\left.\quad+(\eta-w(\xi)) \varphi^{\prime \prime \prime}(\xi)\right]\left(\partial_{x} \xi\right)^{2} \\
\quad-\left[w^{\prime \prime}(\xi) \varphi(\xi)+(\eta-w(\xi)) \varphi^{\prime \prime}(\xi)\right] \partial_{x}^{2} \xi-\varphi^{\prime}(\xi) \partial_{x}^{2} \eta-2 \varphi^{\prime \prime}(\xi) \partial_{x} \xi \partial_{x} \eta \\
\partial_{t}\left(\partial_{x}^{2} \eta\right)=\varphi^{\prime \prime}(\xi)\left(\partial_{x} \xi\right)^{2}+\varphi^{\prime}(\xi) \partial_{x}^{2} \xi \\
\partial_{x}^{2} \xi(0, x)=0, \partial_{x}^{2} \eta(0, x)=w^{\prime \prime}(x) .
\end{array}\right.
$$


Since $\xi\left(\cdot, x_{0}\right) \equiv x_{0}$ and $\eta\left(\cdot, x_{0}\right) \equiv w\left(x_{0}\right)$ by (3.6), we have that $\partial_{x}^{2} \xi\left(\cdot, x_{0}\right)$ and $\partial_{x}^{2} \eta\left(\cdot, x_{0}\right)$ solve the system

$$
\left\{\begin{array}{l}
\partial_{t}\left(\partial_{x}^{2} \xi\left(\cdot, x_{0}\right)\right)=0 \\
\partial_{t}\left(\partial_{x}^{2} \eta\left(\cdot, x_{0}\right)\right)=0 \\
\partial_{x} \xi\left(0, x_{0}\right)=0, \partial_{x} \eta\left(0, x_{0}\right)=w^{\prime \prime}\left(x_{0}\right)
\end{array}\right.
$$

and so

$$
\partial_{x}^{2} \xi\left(t, x_{0}\right) \equiv 0, \quad \partial_{x}^{2} \eta\left(t, x_{0}\right) \equiv w^{\prime \prime}\left(x_{0}\right) .
$$

By (5.10), (5.25), (5.26), (5.35), (5.36), (5.39), (5.40), (5.27), (5.28), (5.41), and (5.42), we have that

$$
\begin{aligned}
\lim _{(s, x) \rightarrow\left(s_{0}, x_{0}\right)^{+}} \partial_{x}^{2} g(s, x)= & -\frac{2 w^{\prime}\left(x_{0}\right) w^{\prime \prime}\left(x_{0}\right)}{\left[1+\left(w^{\prime}\left(x_{0}\right)\right)^{2}\right]^{2}}-\frac{k w^{\prime}\left(x_{0}\right)}{\left[1+\left(w^{\prime}\left(x_{0}\right)\right)^{2}\right]^{2}} \ell_{2}+0 \\
& +\frac{\left[1-\left(w^{\prime}\left(x_{0}\right)\right)^{2}\right] w^{\prime \prime}\left(x_{0}\right)}{\left[1+\left(w^{\prime}\left(x_{0}\right)\right)^{2}\right]^{2}} w^{\prime}\left(x_{0}\right)+\frac{w^{\prime}\left(x_{0}\right) k}{\left[1+\left(w^{\prime}\left(x_{0}\right)\right)^{2}\right]^{2}} \ell_{2} \\
& +\frac{w^{\prime}\left(x_{0}\right) w^{\prime \prime}\left(x_{0}\right)}{1+\left(w^{\prime}\left(x_{0}\right)\right)^{2}}=0
\end{aligned}
$$

and so we can conclude, as before, that $\partial_{x}^{2} g$ exists and is continuous at $\left(s_{0}, x_{0}\right)$.

\section{Acknowledgements}

The authors acknowledge the Center for Nonlinear Analysis (NSF PIRE Grant No. OISE-0967140) where part of this work was carried out. The research of I. Fonseca was partially funded by the National Science Foundation under Grant No. DMS-1411646 and that of G. Leoni under Grant No. DMS-1412095. M.G. Mora acknowledges support by the European Research Council under Grant No. 290888. The authors would like to thank Bob Pego for his helpful insights.

\section{References}

[AFM] Acerbi, E.; Fusco, N.; Morini, M.: Minimality via second variation for a nonlocal isoperimetric problem. Comm. Math. Phys. 322 (2013), 515-557.

[AC] Alt, H.W.; Caffarelli, L.A.: Existence and regularity for a minimum problem with free boundary. J. Reine Angew. Math. 325 (1981), 105-144.

[ACF1] Alt, H.W.; Caffarelli, L.A.; Friedman, A.: Jet flows with gravity. J. Reine Angew. Math. 331 (1982), 58-103.

[ACF2] Alt, H.W.; Caffarelli, L.A.; Friedman, A.: Jets with two fluids. I. One free boundary. Indiana Univ. Math. J. 33 (1984), 213-247. 
[ACF3] Alt, H.W.; Caffarelli, L.A.; Friedman, A.: Jets with two fluids. II. Two free boundaries. Indiana Univ. Math. J. 33 (1984), 367-391.

[AP] Ambrosetti, A.; Prodi, G.: A Primer of Nonlinear Analysis. Cambridge University Press, Cambridge, 1993.

[AL] Arama, D.; Leoni, G.: On a variational approach for water waves. Comm. Partial Differential Equations 37 (2012), 833-874.

[B] Bonacini, M.: Stability of equilibrium configurations for elastic films in two and three dimensions. Adv. Calc. Var. 8 (2015), 117-153.

[BC1] Bonacini, M.; Cristoferi, R.: Local and global minimality issues for a nonlocal isoperimetric problem on $\mathbb{R}^{N}$. Atti Accad. Naz. Lincei Rend. Lincei Mat. Appl. 27 (2016), 37-50.

[BC2] Bonacini, M.; Cristoferi, R.: Local and global minimality results for a nonlocal isoperimetric problem on $\mathbb{R}^{N}$. SIAM J. Math. Anal. 46 (2014), 2310-2349.

[BM] Bonacini, M.; Morini, M.: Stable regular critical points of the Mumford-Shah functional are local minimizers. Ann. Inst. H. Poincaré Anal. Non Linéaire 32 (2015), 533-570.

[BF] Boyer, F.; Fabrie, P.: Mathematical tools for the study of the incompressible Navier-Stokes equations and related models. Applied Mathematical Sciences, 183. Springer, New York, 2013.

[CJK] Caffarelli, L.A.; Jerison, D.; Kenig, C.E.: Global energy minimizers for free boundary problems and full regularity in three dimensions. Noncompact problems at the intersection of geometry, analysis, and topology, 83-97, Contemp. Math., 350, Amer. Math. Soc., Providence, RI, 2004.

[CS] Caffarelli, L.; Salsa, S.: A geometric approach to free boundary problems. Graduate Studies in Mathematics, 68. American Mathematical Society, Providence, RI, 2005.

[CMM] Cagnetti, F.; Mora, M.G.; Morini, M.: A second order minimality condition for the Mumford-Shah functional. Calc. Var. Partial Differential Equations $\mathbf{3 3}$ (2008), 37-74.

[CoSS] Constantin, A.; Sattinger, D.; Strauss, W.: Variational formulations for steady water waves with vorticity. J. Fluid Mech. 548 (2006), 151-163.

[CoS] Constantin, A.; Strauss, W.: Pressure beneath a Stokes wave. Comm. Pure Appl. Math. 63 (2010), 533-557.

[C] Cristoferi, R.: A second order local minimality criterion for the triple junction singularity of the Mumford-Shah functional. ESAIM Control Optim. Calc. Var., to appear. 
[F] Friedman, A.: Variational principles and free-boundary problems. Robert E. Krieger Publishing Company, Inc. Krieger Drive, Malabar, FL, 1988.

[FM] Fusco, N.; Morini, M.: Equilibrium configurations of epitaxially strained elastic films: second order minimality conditions and qualitative properties of solutions. Arch. Ration. Mech. Anal. 203 (2012), 247-327.

[GT] Gilbarg, D.; Trudinger, N.S.: Elliptic Partial Differential Equations of Second Order (2nd edition, Springer, New York, 1984).

[G] M.E. Gurtin: An introduction to continuum mechanics. Mathematics in Science and Engineering, 158. Academic Press Inc., New York-London, 1981.

[KS] D. Kinderlehrer, D.; Stampacchia G.: An introduction to variational inequalities and their applications. Reprint of the 1980 original. Classics in Applied Mathematics, 31. Society for Industrial and Applied Mathematics (SIAM), Philadelphia, PA, 2000.

[MT] Milne-Thomson, L.M.: Theoretical hydrodynamics. 4th ed. The Macmillan Co., New York 1960.

[MM] Mora, M.G.; Morini, M.: Local calibrations for minimizers of the MumfordShah functional with a regular discontinuity set. Ann. Inst. H. Poincaré Anal. Non Linéaire 18 (2001), 403-436.

[PT] Plotnikov, P.I.; Toland, J.F.: Convexity of Stokes waves of extreme form. Arch. Ration. Mech. Anal. 171 (2004), 349-416.

[ST] Shargorodsky, E.; Toland, J.F.: Bernoulli free-boundary problems. Mem. Amer. Math. Soc. 196 (2008), no. 914.

[S] Stokes, G.G.: On the theory of oscillatory waves. Trans. Cambridge Philos. Soc. 8 (1987), 441-455.

[S2] Stokes, G.G.: Considerations relative to the greatest height of oscillatory irrotational waves which can be propagated without change of form. Mathematical and Physical Papers. Vol. I, Cambridge, 1880, pp. 225-228.

[T] Toland, J.F.: Non-existence of global energy minimisers in Stokes waves problems. Discrete Contin. Dyn. Syst. 34 (2014), 3211-3217.

[V] Varvaruca, E.: Bernoulli free-boundary problems in strip-like domains and a property of permanent waves on water of finite depth. Proc. Roy. Soc. Edinburgh Sect. A 138 (2008), 1345-1362.

[VW] Varvaruca, E.; Weiss, G.S.: A geometric approach to generalized Stokes conjectures. Acta Math. 206 (2011), 363-403.

[VW2] Varvaruca, E.; Weiss, G.S.: The Stokes conjecture for waves with vorticity. Ann. Inst. H. Poincaré Anal. Non Linéaire 29 (2012), 861-885. 
[W] Weiss, G.S.: Partial regularity for a minimum problem with free boundary. $J$. Geom. Anal. 9 (1999), 317-326.

[WZ] Weiss, G.S.; Zhang, G.: The second variation of the stream function energy of water waves with vorticity. J. Differential Equations 253 (2012), 2646-2656.

(I. Fonseca) Department of Mathematical Sciences, Carnegie Mellon University, PittsBURGH PA 15213-3890, USA

E-mail address: fonseca@andrew.cmu.edu

(G. Leoni) Department of Mathematical Sciences, Carnegie Mellon University, PittsBURGH PA 15213-3890, USA

E-mail address: giovanni@andrew.cmu.edu

(M.G. Mora) Dipartimento di Matematica, Università di Pavia, via Ferrata 1, 27100 PAVIA, ITALY

E-mail address: mariagiovanna.mora@unipv.it 